\title{
TIMED PARITY GAMES: COMPLEXITY AND ROBUSTNESS
}

\author{
KRISHNENDU CHATTERJEE ${ }^{a}$, THOMAS A. HENZINGER $^{b}$, AND VINAYAK S. PRABHU $^{c}$ \\ ${ }^{a, b}$ IST Austria (Institute of Science and Technology, Austria) \\ e-mail address: \{krish.chat,tah\}@ist.ac.at \\ ${ }^{c}$ University of Porto \\ e-mail address: vinayak@eecs.berkeley.edu
}

\begin{abstract}
We consider two-player games played in real time on game structures with clocks where the objectives of players are described using parity conditions. The games are concurrent in that at each turn, both players independently propose a time delay and an action, and the action with the shorter delay is chosen. To prevent a player from winning by blocking time, we restrict each player to play strategies that ensure that the player cannot be responsible for causing a zeno run. First, we present an efficient reduction of these games to turn-based (i.e., not concurrent) finite-state (i.e., untimed) parity games. Our reduction improves the best known complexity for solving timed parity games. Moreover, the rich class of algorithms for classical parity games can now be applied to timed parity games. The states of the resulting game are based on clock regions of the original game, and the state space of the finite game is linear in the size of the region graph.

Second, we consider two restricted classes of strategies for the player that represents the controller in a real-time synthesis problem, namely, limit-robust and bounded-robust winning strategies. Using a limit-robust winning strategy, the controller cannot choose an exact real-valued time delay but must allow for some nonzero jitter in each of its actions. If there is a given lower bound on the jitter, then the strategy is bounded-robust winning. We show that exact strategies are more powerful than limit-robust strategies, which are more powerful than bounded-robust winning strategies for any bound. For both kinds of robust strategies, we present efficient reductions to standard timed automaton games. These reductions provide algorithms for the synthesis of robust real-time controllers.
\end{abstract}

\section{INTRODUCTION}

Timed automata [AD94 are models of real-time systems in which states consist of discrete locations and values for real-time clocks. The transitions between locations are dependent on the clock values. Timed automaton games, introduced in [MPS95], and explored further in $\mathrm{dAFH}^{+} 03, \mathrm{AdAF} 05, \mathrm{CDF}^{+} 05, \mathrm{FTM02b}$, FTM02a (amongst others), are played by two players on timed automata, e.g., a "controller" and a "plant" for modeling real-time

1998 ACM Subject Classification: F.4.1.

Key words and phrases: Timed Automata, Timed Parity Games, Robust Timed Control.

This research was supported in part by the NSF grants CCR-0132780, CNS-0720884, and CCR-0225610, and by the European COMBEST project. 


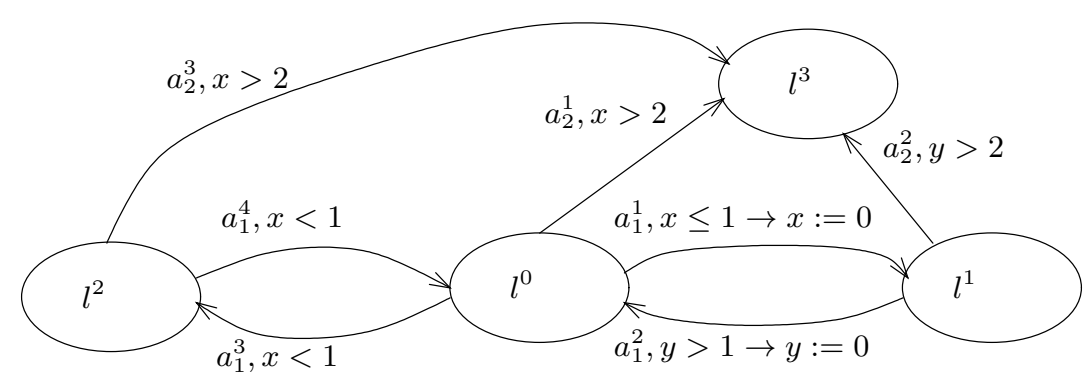

Figure 1: A timed automaton game $\mathcal{T}$.

controller synthesis problems. We consider timed automaton games with $\omega$-regular objectives specified as parity conditions. The class of $\omega$-regular objectives can express all safety and liveness specifications that arise in the synthesis and verification of reactive systems, and parity conditions are a canonical form to express $\omega$-regular objectives [Tho97. The construction of a winning strategy for player 1 in such games corresponds to the controllersynthesis problem for real-time systems [DM02, MPS95, WH91] with respect to achieving a desired $\omega$-regular objective.

Timed automaton games proceed in an infinite sequence of rounds. In each round, both players simultaneously propose moves, with each move consisting of an action and a time delay after which the player wants the proposed action to take place. Of the two proposed moves, the move with the shorter time delay "wins" the round and determines the next state of the game. Let a set $\Phi$ of runs be the desired objective for player 1 . Then player 1 has a winning strategy for $\Phi$ if it has a strategy to ensure that, no matter what player 2 does, one of the following two conditions holds: (1) time diverges and the resulting run belongs to $\Phi$, or (2) time does not diverge but player-1's moves are chosen only finitely often (and thus it is not to be blamed for the convergence of time) dAFH $\mathrm{dA3}^{+}$, HP06. This definition of winning is equivalent to restricting both players to play according to receptive strategies AH97, SGSAL98, which do not allow a player to win by blocking time.

In timed automaton games, there are cases where a player can win by proposing a certain strategy of moves, but where moves that deviate in the timing by an arbitrarily small amount from the winning strategy result in a strategy that does not ensure winning any more. If this is the case, then the synthesized controller needs to work with infinite precision in order to achieve the control objective. As this requirement is unrealistic, we propose two notions of robust winning strategies. In the first robust model, each move of player 1 (the "controller") must allow some jitter when the action of the move is taken. The jitter may be arbitrarily small, but it must be greater than 0 . We call such strategies limit-robust. In the second robust model, we give a lower bound on the jitter, i.e., every move of player 1 must allow for a fixed jitter, which is specified as a parameter of the game. We call these strategies bounded-robust. The strategies of player 2 (the "plant") are left unrestricted (apart from being receptive). We show that (1) general strategies are strictly more powerful than limit-robust strategies; and (2) limit-robust strategies are strictly more powerful than bounded-robust strategies for any lower bound on the jitter, i.e., there are games in which player 1 can win with a limit-robust strategy, but there does not exist any nonzero bound on the jitter for which player 1 can win with a bounded-robust strategy. The following example illustrates this issue. 
Example 1.1. Consider the timed automaton $\mathcal{T}$ in Fig. 1, The edges denoted $a_{1}^{k}$ for $k \in\{1,2,3,4\}$ are controlled by player 1 , and the edges denoted $a_{2}^{j}$ for $j \in\{1,2,3\}$ are controlled by player 2. The objective of player 1 is $\square\left(\neg l^{3}\right)$, i.e., to avoid the location $l^{3}$. The important part of the automaton is the cycle $l^{0}, l^{1}$. The only way to avoid $l^{3}$ in a time divergent run is to cycle between $l^{0}$ and $l^{1}$ infinitely often. In addition, player 1 may choose to also cycle between $l^{0}$ and $l^{2}$, but that does not help (or harm) it. Due to strategies being required to be receptive, player 1 cannot just cycle between $l^{0}$ and $l^{2}$ forever, it must also cycle between $l^{0}$ and $l^{1}$; that is, to satisfy $\square\left(\neg l^{3}\right)$ player 1 must ensure $\left(\square \diamond l^{0}\right) \wedge\left(\square \diamond l^{1}\right)$, where $\square \diamond$ denotes "infinitely often". But note that player 1 may cycle between $l^{0}$ and $l^{2}$ any finite number of times as it wants between an $l^{0}, l^{1}$ cycle.

In our analysis below, we omit such $l^{0}, l^{2}$ cycles for simplicity. Let the game start from the location $l^{0}$ at time 0 , and let $l^{1}$ be visited at time $t^{0}$ for the first time. Also, let $\alpha^{j}$ denote the difference between times when $l^{0}$ is visited for the $(j+1)$-th time, and when $l^{1}$ is visited for the $j$-th time. We can have at most 1 time unit between two successive visits to $l^{0}$, and we must have strictly more than 1 time unit elapse between two successive visits to $l^{1}$. Thus, $\alpha^{j}$ must be in a strictly decreasing sequence. Also, for player 1 to cycle between $l^{0}$ and $l^{1}$ infinitely often, we must have $\alpha^{j} \geq 0$ for all $j$ as the $(j+1)$-th $a_{1}^{1}$ transition must always happen after the $j$-th $a_{1}^{2}$ transition. Consider any bounded-robust strategy. Since the jitter is some fixed $\varepsilon_{\mathrm{j}}$, for any strategy of player 1 that tries to cycle between $l^{0}$ and $l^{1}$, there are executions where the transition labeled $a_{1}^{1}$ is taken when $x$ is less than or equal to $1-\varepsilon_{\mathrm{j}}$, and the transition labeled $a_{1}^{2}$ is taken when $y$ is greater than 1 . This means that there are executions where $\alpha^{j}$ decreases by at least $\varepsilon_{\mathrm{j}}$ in each cycle. But, this implies that we cannot have an infinite decreasing sequence of $\alpha^{j}$ 's for any $\varepsilon_{\mathrm{j}}$ and for any starting value of $t^{0}$.

With a limit-robust strategy, however, player 1 can cycle between the two locations infinitely often, provided that the starting value of $x$ is strictly less than 1 . This is because at each step of the game, player 1 can choose moves that are such that the clocks $x$ and $y$ are closer and closer to 1 . A general strategy allows player 1 to win even when the starting value of $x$ is 1. The details will be presented later in Example 4.9 in Subsection 4.2.

Contributions. Our contributions are two-fold: we present improved complexity results to solve timed automaton parity games, and we present two notions of robust winning in timed automaton parity games and present solutions of them.

Improved complexity. We first show that timed automaton parity games can be reduced to classical turn-based finite-state (untimed) parity games. Even though the timed games are concurrent, in that in each turn both players simultaneously propose moves before one of the moves is chosen, our reduction to the untimed finite-state game is turn-based. The concurrency in timed games is limited (only in proposal of time), and we exploit this in the reduction to obtain turn-based games. In general the reduction of concurrent games to turn-based games is only known for Büchi and coBüchi objectives (and only for qualitative analysis) [JKH02]. The turn-based game we obtain as result of the reduction has a state space that is linear in the number of clock regions. There is a rich literature of algorithms as well much ongoing research to solve finite-state turn-based parity games, and our reduction allows all these algorithms to be used to solve timed automaton parity games. A solution for timed automaton games with parity objectives was already presented in $\mathrm{dAFH}^{+} 03$ and the solution works in

$$
O\left(\left(M \cdot|C| \cdot\left|A_{1}\right| \cdot\left|A_{2}\right|\right)^{2} \cdot\left(\left|S_{\mathrm{Reg}}^{*}\right|\right)^{d+2}\right)
$$


time, where $M$ is the maximum constant in the timed automaton; $C$ is the set of clocks; $A_{i}$ is the set of player- $i$ edges; $L$ is the set of locations; $d$ is the number of priorities in the parity index function; and $S_{\mathrm{Reg}}^{*}$ is the set of states in the region graph of the timed automaton expanded to handle receptiveness and we have $\left|S_{\mathrm{Reg}}^{*}\right|=\left|S_{\mathrm{Reg}}\right| \cdot 32 \cdot|C| \cdot d$, where $S_{\mathrm{Reg}}$ is the set of regions of the timed automaton (which is bounded by $|L| \cdot \prod_{x \in C}\left(c_{x}+1\right) \cdot|C| ! \cdot 4^{|C|}$, with $c_{x}$ being the maximum constant that clock $x$ is compared to in the timed automaton game). We show that timed automaton games can be solved in

$$
O\left(\left(\left|S_{\mathrm{Reg}}^{*}\right| \cdot\left(\left|A_{1}\right|+\left|A_{2}\right|\right)\right) \cdot\left(\left|S_{\mathrm{Reg}}^{*}\right| \cdot 8\right)^{\frac{d+2}{3}+\frac{1}{2}}\right)
$$

time. Our reduction has two steps: first we show that certain restrictions can be applied to strategies without changing the winning set; then we show that the timed game with clocks under the strategy restrictions can be transformed into a finite-state turn-based game with $8 \cdot\left|S_{\text {Reg }}^{*}\right|$ states, $O\left(\left|S_{\text {Reg }}^{*}\right| \cdot\left(\left|A_{1}\right|+\left|A_{2}\right|\right)\right)$ edges, and $d+2$ priorities. Our improved complexity follows from the above reduction, and the fact that a finite-state turn-based parity game with $m$ edges, $n$ states and $d$ parity indices can be solved in $O\left(m \cdot n^{\frac{d}{3}+\frac{1}{2}}\right)$ time [Sch07. The restriction to receptive strategies is handled by our reduction with the following two modifications: (1) the number of regions of the timed automaton parity game needs to be enlarged by a factor of $32 \cdot|C| \cdot d$, and (2) the number of indices of the parity function needs to be increased by 2 . The modifications are similar to those in $\left[\mathrm{dAFH}^{+} 03\right]$.

Robust winning. Second, we show that timed automaton games with limit-robust and bounded-robust strategies can be solved by reductions to general timed automaton games (with exact strategies). In the reduction for limit-robust games, the jitter is controlled by player 1 , as the jitter is only required to be greater than 0 , with no other restriction. For bounded-robust games, the jitter is controlled by player 2 , as there is an uncertainty interval of constant length $\varepsilon_{\mathrm{j}}$ (which is fixed for the game). The reduction for the limit-robust case is obtained by changing the winning condition so that moves are only to states where all the clock values are non-integral. The reduction for the bounded-robust case is by a syntactic transformation of the game graph. The limit-robust game can be solved in time

$$
O\left(\left(\left|S_{\operatorname{Reg}}^{*}\right| \cdot\left(\left|A_{1}\right|+\left|A_{2}\right|\right)\right) \cdot\left(\left|S_{\operatorname{Reg}}^{*}\right| \cdot 16\right)^{\frac{d+2}{3}+\frac{1}{2}}\right) .
$$

Given a rational valued jitter of $\varepsilon=\frac{\varepsilon_{n}}{\varepsilon_{d}}$, the bounded-robust game can be solved in time

$$
O\left(\left(\left|S_{\mathrm{Reg}}^{*}\right| \cdot\left|A_{1}\right|^{2} \cdot\left|A_{2}\right| \cdot|C| \cdot \varepsilon_{n} \cdot \varepsilon_{d}^{|C|+2}\right) \cdot\left(\left|S_{\mathrm{Reg}}^{*}\right| \cdot 32 \cdot|C| \cdot\left|A_{1}\right| \cdot \varepsilon_{n} \cdot \varepsilon_{d}^{|C|+2}\right)^{\frac{d+2}{3}+\frac{1}{2}}\right) .
$$

The reductions provide algorithms for synthesizing robust controllers for real-time systems, where the controller is guaranteed to achieve the control objective even if its time delays are subject to jitter. The question of the existence of a non-zero jitter for which a game can be won with a bounded-robust strategy remains open.

Comparison to the preliminary version [CHP08]. Our present submission extends and improves upon the results of [CHP08]. The state space of the finite game of the reduction in [CHP08] had size $O\left(\left|S_{\mathrm{Reg}}^{*}\right| \cdot|C| \cdot M \cdot\left|A_{1}\right|\right)$; the present finite-state game has size $O\left(\left|S_{\mathrm{Reg}}^{*}\right|\right)$. The correctness proof of the present reduction is based on several new non-trivial results that are not present in [CHP08. 
Related work. Timed automaton games have been explored before for controller synthesis, e.g, in [PAMS98, DM02, MPS95, WH91]. In most previous work, time-divergence has not been handled properly. For example, the formulations of [PAMS98, FTM02b, JT07, Tri09] assume that the syntactic structure of the game is such that it is not possible for the players to block time. The work of [AH97] looks at safety objectives, and correctly requires that a player might not stay safe simply by blocking time. It however also requires that the controller achieve its objective even if the opponent blocks time, and hence is deficient for reachability objectives. In DM02 the authors require player 1 to always allow player 2 moves, thus, in particular, player 2 can foil a reachability objective of player 1 by blocking time. The strategies of player 1 are also assumed to be region strategies by definition, that is, player-1 strategies can only specify regions in its moves (and not an exact desired state), and can only be dependent on the history of the observed regions in a play (and not on the history of exact states in a play). A solution for timed automaton games with receptive strategies and parity objectives was first presented in $\mathrm{dAFH}^{+} 03$, where the solution is obtained by first demonstrating that the winning set can be characterized by a $\mu$-calculus fixpoint expression, and then showing that only unions of clock regions arise in its fixpoint iteration.

It has been recognized by many researchers that an important shortcoming of timed automata is that clock values are assumed to be available as real numbers with infinite precision. Our notion of bounded-robustness is closely related to the Almost-ASAP semantics of [WDR05]. The work there is done in a one-player setting where the controller is already known, and one wants the know if the composition of the controller and the system satisfies a safety property in the presence of bounded jitter and observation delay. A similar model for hybrid automata is considered in [AT04]. The solution for the existence of bounded jitter and observation delay for which a timed system stays safe is presented in [WDMR04. Various models of robustness in timed automata (the one-player case) are also considered in ATM05, BMR08, GHJ97, HR00].

Outline. In Section 2 we present the definitions of timed game structures, objectives, strategies, and review some basic results for timed automaton games. In Section 3 we present a restriction of strategies of the two players, which does not change the winning set, and which allows an efficient reduction to finite-state turn-based games. We also derive the complexity bound obtained from the new reduction to finite-state games. In Section 4 we define limit-robust and bounded-robust strategies, and show how winning sets for both can be computed by reductions to timed automaton games.

\section{Timed Games}

In this section we present the definitions of timed game structures, runs, objectives, and strategies in timed game structures.

Timed game structures. A timed game structure is a tuple $\mathcal{G}=\left\langle S, A_{1}, A_{2}, \Gamma_{1}, \Gamma_{2}, \delta\right\rangle$ with the following components.

- $S$ is a set of states.

- $A_{1}$ and $A_{2}$ are two disjoint sets of actions for players 1 and 2, respectively. We assume that $\left\{\perp_{1}, \perp_{2}, \perp_{*}\right\} \cap A_{i}=\emptyset$, and write $A_{1}^{\perp}$ for $A_{1} \cup\left\{\perp_{1}, \perp_{*}\right\}$, and $A_{2}^{\perp}$ for $A_{2} \cup\left\{\perp_{2}\right\}$. The set of moves for player $i$ is $M_{i}=\mathbb{R}_{\geq 0} \times A_{i}^{\perp}$. Intuitively, a move $\left\langle\Delta, a_{i}\right\rangle$ by player $i$ indicates a waiting period of $\Delta$ time units followed by a discrete transition labeled with 
action $a_{i}$. The move $\left\langle\Delta, \perp_{i}\right\rangle$ is used to represent the move where player- $i$ just lets time elapse for $\Delta$ time units without taking any of the discrete actions from $A_{i}$. The action $\perp_{*}$ is used to represent the fact that player 1 is relinquishing control to player 2 in the given stage of the game.

- $\Gamma_{i}: S \mapsto 2^{M_{i}} \backslash \emptyset$ are two move assignments. At every state $s$, the set $\Gamma_{i}(s)$ contains the moves that are available to player $i$. We require that $\left\langle 0, \perp_{i}\right\rangle \in \Gamma_{i}(s)$ for all states $s \in S$ and $i \in\{1,2\}$. Intuitively, $\left\langle 0, \perp_{i}\right\rangle$ is a time-blocking stutter move.

- $\delta: S \times\left(M_{1} \cup M_{2}\right) \mapsto S$ is the transition function. We require that for all time delays $\Delta, \Delta^{\prime} \in \mathbb{R}_{\geq 0}$ with $\Delta^{\prime} \leq \Delta$, and all actions $a_{i} \in A_{i}^{\perp}$, we have

(i) $\left\langle\Delta, a_{i}\right\rangle \in \Gamma_{i}(s)$ iff both $\left\langle\Delta^{\prime}, \perp_{i}\right\rangle \in \Gamma_{i}(s)$ and $\left\langle\Delta-\Delta^{\prime}, a_{i}\right\rangle \in \Gamma_{i}\left(\delta\left(s,\left\langle\Delta^{\prime}, \perp_{i}\right\rangle\right)\right)$; and

(ii) if $\delta\left(s,\left\langle\Delta^{\prime}, \perp_{i}\right\rangle\right)=s^{\prime}$ and $\delta\left(s^{\prime},\left\langle\Delta-\Delta^{\prime}, a_{i}\right\rangle\right)=s^{\prime \prime}$, then $\delta\left(s,\left\langle\Delta, a_{i}\right\rangle\right)=s^{\prime \prime}$.

The game proceeds as follows. If the current state of the game is $s$, then both players simultaneously propose moves $\left\langle\Delta_{1}, a_{1}\right\rangle \in \Gamma_{1}(s)$ and $\left\langle\Delta_{2}, a_{2}\right\rangle \in \Gamma_{2}(s)$. If $a_{1} \neq \perp_{*}$, the move with the shorter duration "wins" in determining the next state of the game. If both moves have the same duration, then the next state is chosen non-deterministically. If $a_{1}=\perp_{*}$, then the move of player 2 determines the next state, regardless of $\Delta_{1}$. We give this special power to player 1 for modeling convenience as (1) the controller always has the option of letting the state evolve in a controller-plant framework, without always having to provide inputs to the plant, and (2) it allows a natural model for systems where controller actions are disabled in certain modes]. Formally, we define the joint destination function $\delta_{\text {jd }}: S \times M_{1} \times M_{2} \mapsto 2^{S}$ by

$$
\delta_{\text {jd }}\left(s,\left\langle\Delta_{1}, a_{1}\right\rangle,\left\langle\Delta_{2}, a_{2}\right\rangle\right)= \begin{cases}\left\{\delta\left(s,\left\langle\Delta_{1}, a_{1}\right\rangle\right)\right\} & \text { if } \Delta_{1}<\Delta_{2} \text { and } a_{1} \neq \perp_{*} \\ \left\{\delta\left(s,\left\langle\Delta_{2}, a_{2}\right\rangle\right)\right\} & \text { if } \Delta_{2}<\Delta_{1} \text { or } a_{1}=\perp_{*} \\ \left\{\delta\left(s,\left\langle\Delta_{2}, a_{2}\right\rangle\right), \delta\left(s,\left\langle\Delta_{1}, a_{1}\right\rangle\right)\right\} & \text { if } \Delta_{2}=\Delta_{1} \text { and } a_{1} \neq \perp_{*} .\end{cases}
$$

The time elapsed when the moves $m_{1}=\left\langle\Delta_{1}, a_{1}\right\rangle$ and $m_{2}=\left\langle\Delta_{2}, a_{2}\right\rangle$ are proposed is given by

$$
\operatorname{delay}\left(m_{1}, m_{2}\right)= \begin{cases}\min \left(\Delta_{1}, \Delta_{2}\right) & \text { if } a_{1} \neq \perp_{*} \\ \Delta_{2} & \text { if } a_{1}=\perp_{*}\end{cases}
$$

The boolean predicate blame $e_{i}\left(s, m_{1}, m_{2}, s^{\prime}\right)$ indicates whether player $i$ is "responsible" for the state change from $s$ to $s^{\prime}$ when the moves $m_{1}$ and $m_{2}$ are proposed. Denoting the opponent of player $i$ by $\sim i=3-i$, for $i \in\{1,2\}$, we define

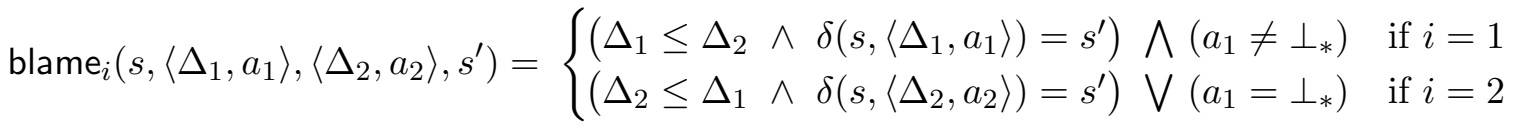

Runs. A run $r=s_{0},\left\langle m_{1}^{0}, m_{2}^{0}\right\rangle, s_{1},\left\langle m_{1}^{1}, m_{2}^{1}\right\rangle, \ldots$ of the timed game structure $\mathcal{G}$ is an infinite sequence such that $s_{k} \in S$ and $m_{i}^{k} \in \Gamma_{i}\left(s_{k}\right)$ and $s_{k+1} \in \delta_{\text {jd }}\left(s_{k}, m_{1}^{k}, m_{2}^{k}\right)$ for all $k \geq 0$ and $i \in\{1,2\}$. For $k \geq 0$, let time $(r, k)$ denote the "time" at position $k$ of the run, namely, $\operatorname{time}(r, k)=\sum_{j=0}^{k-1} \operatorname{delay}\left(m_{1}^{j}, m_{2}^{j}\right)$ (we let time $(r, 0)=0$ ). By $r[k]$ we denote the $(k+1)$-th state $s_{k}$ of $r$. The run prefix $r[0 . . k]$ is the finite prefix of the run $r$ that ends in the state $s_{k}$. Let Runs be the set of all runs of $\mathcal{G}$, and let FinRuns be the set of run prefixes.

Objectives. An objective for the timed game structure $\mathcal{G}$ is a set $\Phi \subseteq$ Runs of runs. We will be interested in parity objectives. Parity objectives are canonical forms for $\omega$-regular properties that can express all commonly used specifications that arise in verification.

\footnotetext{
${ }^{1}$ We illustrate the usefulness of $\perp_{*}$ in Example 2.2. The results of this paper do not change if $\perp_{*}$ is not present in the framework.
} 
Let $\Omega: S \mapsto\{0, \ldots, k-1\}$ be a parity index function. The parity objective for $\Omega$ requires that the maximal index visited infinitely often be even. Formally, let InfOften $(\Omega(r))$ denote the set of indices visited infinitely often along a run $r$. Then the parity objective defines the following set of runs: Parity $(\Omega)=\{r \mid \max (\operatorname{InfOften}(\Omega(r)))$ is even $\}$. A timed game structure $\mathcal{G}$ together with the index function $\Omega$ constitute a parity timed game (of order $k$ ) in which the objective of player 1 is $\operatorname{Parity}(\Omega)$.

Strategies. A strategy for a player is a recipe that specifies how to extend a run. Formally, a strategy $\pi_{i}$ for player $i \in\{1,2\}$ is a function $\pi_{i}$ that assigns to every run prefix $r[0 . . k]$ a move $m_{i}$ in the set of moves available to player $i$ at the state $r[k]$. For $i \in\{1,2\}$, let $\Pi_{i}$ be the set of strategies for player $i$. Given two strategies $\pi_{1} \in \Pi_{1}$ and $\pi_{2} \in \Pi_{2}$, the set of possible outcomes of the game starting from a state $s \in S$ is the set of possible runs denoted by Outcomes $\left(s, \pi_{1}, \pi_{2}\right)$.

Receptive strategies. We will be interested in strategies that are meaningful (in the sense that they do not block time). To define them formally we first present the following two sets of runs.

- A run $r$ is time-divergent if $\lim _{k \rightarrow \infty} \operatorname{time}(r, k)=\infty$. We denote by Timediv the set of all time-divergent runs.

- The set Blameless B $_{i} \subseteq$ Runs consists of the set of runs in which player $i$ is responsible only for finitely many transitions. A run $s_{0},\left\langle m_{1}^{0}, m_{2}^{0}\right\rangle, s_{1},\left\langle m_{1}^{1}, m_{2}^{1}\right\rangle, \ldots$ belongs to the set Blameless $i$, for $i=\{1,2\}$, if there exists a $k \geq 0$ such that for all $j \geq k$, we have $\neg$ blame $_{i}\left(s_{j}, m_{1}^{j}, m_{2}^{j}, s_{j+1}\right)$.

A strategy $\pi_{i}$ is receptive if for all strategies $\pi_{\sim i}$, all states $s \in S$, and all runs $r \in$ Outcomes $\left(s, \pi_{1}, \pi_{2}\right)$, either $r \in$ Timediv or $r \in$ Blameless $_{i}$. Thus, no matter what the opponent does, a receptive strategy of player $i$ cannot be responsible for blocking time. Strategies that are not receptive are not physically meaningful. A timed game structure $\mathcal{G}$ is well-formed if both players have receptive strategies. We restrict our attention to well-formed timed game structures. We denote $\Pi_{i}^{R}$ to be the set of receptive strategies for player $i$. Note that for $\pi_{1} \in \Pi_{1}^{R}, \pi_{2} \in \Pi_{2}^{R}$, we have Outcomes $\left(s, \pi_{1}, \pi_{2}\right) \subseteq$ Timediv.

Winning sets. Given an objective $\Phi$, let $\operatorname{WinTimeDiv}_{1}^{\mathcal{G}}(\Phi)$ denote the set of states $s$ in $\mathcal{G}$ such that player 1 has a receptive strategy $\pi_{1} \in \Pi_{1}^{R}$ such that for all receptive strategies $\pi_{2} \in \Pi_{2}^{R}$, we have Outcomes $\left(s, \pi_{1}, \pi_{2}\right) \subseteq \Phi$. The strategy $\pi_{1}$ is said to be a winning strategy. In computing the winning sets, we shall quantify over all strategies, but modify the objective to take care of time divergence. Given an objective $\Phi$, let

$$
\operatorname{TimeDivBl}_{1}(\Phi)=(\text { Timediv } \cap \Phi) \cup\left(\text { Blameless }_{1} \backslash \text { Timediv }\right)
$$

i.e., TimeDivBl$I_{1}(\Phi)$ denotes the set of runs such that either time diverges and $\Phi$ holds, or else time converges and player 1 is not responsible for time to converge. Let $\operatorname{Win}_{1}^{\mathcal{G}}(\Phi)$ be the set of states in $\mathcal{G}$ such that for all $s \in \operatorname{Win}_{1}^{\mathcal{G}}(\Phi)$, player 1 has a (possibly non-receptive) strategy $\pi_{1} \in \Pi_{1}$ such that for all (possibly non-receptive) strategies $\pi_{2} \in \Pi_{2}$, we have Outcomes $\left(s, \pi_{1}, \pi_{2}\right) \subseteq \Phi$. The strategy $\pi_{1}$ is said to be winning for the non-receptive game. The following result establishes the connection between Win and WinTimeDiv sets.

Theorem 2.1 ([HP06]). For all well-formed timed game structures $\mathcal{G}$, and for all $\omega$-regular objectives $\Phi$, we have $\operatorname{Win}_{1}^{\mathcal{G}}\left(\operatorname{TimeDivBl}_{1}(\Phi)\right)=\operatorname{WinTimeDiv}_{1}^{\mathcal{G}}(\Phi)$.

We now define a special class of timed game structures, namely, timed automaton games. 
Timed automaton games. Timed automata AD94 suggest a finite syntax for specifying infinite-state timed game structures. A timed automaton game is a tuple $\mathcal{T}=$ $\left\langle L, C, A_{1}, A_{2}, E, \gamma\right\rangle$ with the following components:

- $L$ is a finite set of locations.

- $C$ is a finite set of clocks.

- $A_{1}$ and $A_{2}$ are two disjoint sets of actions for players 1 and 2, respectively.

- $E \subseteq L \times\left(A_{1} \cup A_{2}\right) \times$ Constr $(C) \times L \times 2^{C}$ is the edge relation, where the set Constr $(C)$ of clock constraints is generated by the grammar

$$
\theta::=x \leq d|d \leq x| \neg \theta \mid \theta_{1} \wedge \theta_{2}
$$

for clock variables $x \in C$ and nonnegative integer constants $d$.

For an edge $e=\left\langle l, a_{i}, \theta, l^{\prime}, \lambda\right\rangle$, the clock constraint $\theta$ acts as a guard on the clock values which specifies when the edge $e$ can be taken, and by taking the edge $e$, the clocks in the set $\lambda \subseteq C$ are reset to 0 . We require that for all edges $\left\langle l, a_{i}, \theta^{\prime}, l^{\prime}, \lambda^{\prime}\right\rangle \neq\left\langle l, a_{i}^{\prime}, \theta^{\prime \prime}, l^{\prime \prime}, \lambda^{\prime \prime}\right\rangle \in E$, we have $a_{i} \neq a_{i}^{\prime}$. This requirement ensures that a state and a move together uniquely determine a successor state.

- $\gamma: L \mapsto$ Constr $(C)$ is a function that assigns to every location an invariant for both players. All clocks increase uniformly at the same rate. When at location $l$, each player $i$ must propose a move out of $l$ before the invariant $\gamma(l)$ expires. Thus, the game can stay at a location only as long as the invariant is satisfied by the clock values.

A clock valuation is a function $\kappa: C \mapsto \mathbb{R}_{\geq 0}$ that maps every clock to a nonnegative real. The set of all clock valuations for $C$ is denoted by $K(C)$. Given a clock valuation $\kappa \in K(C)$ and a time delay $\Delta \in \mathbb{R}_{\geq 0}$, we write $\kappa+\Delta$ for the clock valuation in $K(C)$ defined by $(\kappa+\Delta)(x)=\kappa(x)+\Delta$ for all clocks $x \in C$. For a subset $\lambda \subseteq C$ of the clocks, we write $\kappa[\lambda:=0]$ for the clock valuation in $K(C)$ defined by $(\kappa[\lambda:=0])(x)=0$ if $x \in \lambda$, and $(\kappa[\lambda:=0])(x)=\kappa(x)$ if $x \notin \lambda$. A clock valuation $\kappa \in K(C)$ satisfies the clock constraint $\theta \in \operatorname{Constr}(C)$, written $\kappa \models \theta$, if the condition $\theta$ holds when all clocks in $C$ take on the values specified by $\kappa$. A state $s=\langle l, \kappa\rangle$ of the timed automaton game $\mathcal{T}$ is a location $l \in L$ together with a clock valuation $\kappa \in K(C)$ such that the invariant at the location is satisfied, that is, $\kappa=\gamma(l)$. We let $S$ be the set of all states of $\mathcal{T}$.

In a state, each player $i$ proposes a time delay allowed by the invariant map $\gamma$, together either with the action $\perp_{i}$ (player 1 can also propose $\perp_{*}$ ), or with an action $a_{i} \in A_{i}$ such that an edge labeled $a_{i}$ is enabled after the proposed time delay. We require that for all states $s=\langle l, \kappa\rangle$, either

(a) $\kappa+\Delta \models \gamma(l)$ for all $\Delta \in \mathbb{R}_{\geq 0}$, or

(b) there exist a time delay $\Delta \in \mathbb{R}_{\geq 0}$ and an edge $\left\langle l, a_{2}, \theta, l^{\prime}, \lambda\right\rangle \in E$ such that

(1) $a_{2} \in A_{2}$ and

(2) $\kappa+\Delta \models \theta$ and for all $0 \leq \Delta^{\prime} \leq \Delta$, we have $\kappa+\Delta^{\prime} \models \gamma(l)$, and

(3) $(\kappa+\Delta)[\lambda:=0] \models \gamma\left(l^{\prime}\right)$.

Informally, these conditions ensure that, for a legal state, either the invariant at the location is satisfied at all time points in the future; or there is some time point in the future at which a discrete action can be taken by the plant (with the location invariant being satisfied up to that time point). This requirement is necessary (but not sufficient) for well-formedness of the game.

The timed automaton game $\mathcal{T}$ defines a timed game structure $\llbracket \mathfrak{T} \rrbracket=\left\langle S, A_{1}, A_{2}, \Gamma_{1}, \Gamma_{2}, \delta\right\rangle$ as follows: 


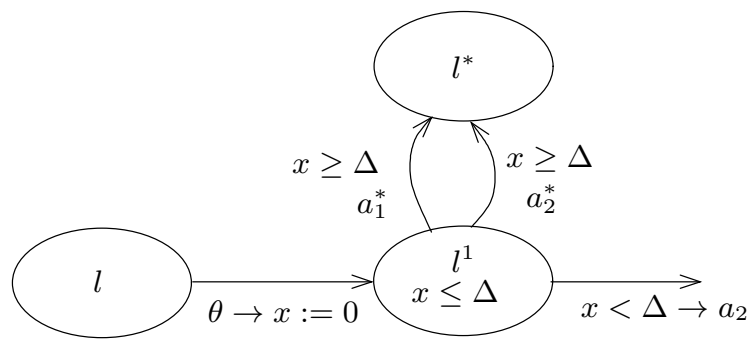

Figure 2: Player-1 actions disabled for $\Delta$ time units $\left(\perp_{*}\right.$ absent in model).

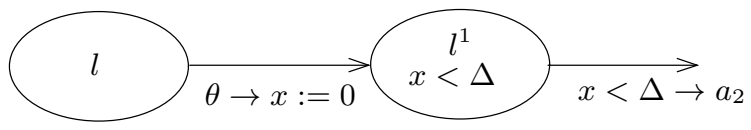

Figure 3: Player-1 actions disabled for $\Delta$ time units $\left(\perp_{*}\right.$ present in model $)$.

- $S=\{\langle l, \kappa\rangle \mid l \in L$ and $\kappa(l)$ satisfies $\gamma(l)\}$.

- For $i \in\{1,2\}$, the set $\Gamma_{i}(\langle l, \kappa\rangle)$ contains the following elements:

(a) $\left\langle\Delta, \perp_{i}\right\rangle$ if for all $0 \leq \Delta^{\prime} \leq \Delta$, we have $\kappa+\Delta^{\prime} \models \gamma(l)$.

(b) $\left\langle\Delta, a_{i}\right\rangle$ if for all $0 \leq \Delta^{\prime} \leq \Delta$, we have $\kappa+\Delta^{\prime} \models \gamma(l), a_{i} \in A_{i}$, and there exists an edge $\left\langle l, a_{i}, \theta, l^{\prime}, \lambda\right\rangle \in E$ such that $\kappa+\Delta \models \theta$.

(c) $\left\langle 0, \perp_{*}\right\rangle$ if $i=1$.

- The transition function $\delta$ is specified by:

(a) $\delta\left(s,\left\langle\Delta, \perp_{*}\right\rangle\right)=s$

(b) $\delta\left(\langle l, \kappa\rangle,\left\langle\Delta, \perp_{i}\right\rangle\right)=\langle l, \kappa+\Delta\rangle$.

(c) $\delta\left(\langle l, \kappa\rangle,\left\langle\Delta, a_{i}\right\rangle\right)=\left\langle l^{\prime},(\kappa+\Delta)[\lambda:=0]\right\rangle$ for the unique edge $\left\langle l, a_{i}, \theta, l^{\prime}, \lambda\right\rangle \in E$ with $\kappa+\Delta \models \theta$.

The timed game structure $\llbracket \mathfrak{T} \rrbracket$ is not necessarily well-formed, because it may contain cycles along which time cannot diverge. Well-formedness of timed automaton games can be checked in EXPTIME [HP06]. We restrict our focus to well-formed timed automaton games in this paper.

Example 2.2 (Utility of the action $\perp_{*}$ ). Suppose we did not have the action $\perp_{*}$. Then in every timed game, we need to require that from every location, there needs to be an outgoing player-1 edge before the invariant of the location expires; this requirement needs to be present as now player 1 cannot simply relinquish active control to player 2 .

Consider a timed automaton game $\mathcal{T}$ with a location $l$ in which we want to model the following. We want that if a clock condition $\theta$ is met at location $l$, then the moves of the controller (player 1) are disabled for the next $\Delta$ time units (the disabled time interval is right open); and that player 2 (the plant) is to take a particular action $a_{2}^{\prime}$ within these $\Delta$ time units. Let $x$ be a clock used just for guarding the $\Delta$ condition. The system can be modeled as in Figure 2. The incoming player-1 and player-2 edges to $l^{1}$ have the same guard and reset condition. Without the $\perp_{*}$ action, we need special player-1 and player-2 actions $a_{1}^{*}$ and $a_{2}^{*}$ which go to a dummy sink accepting location from the location $l^{1}$. The objective $\Phi$ of player 1 also needs to be modified to $\Phi \wedge\left(\neg \diamond l^{*}\right)$ (where $\diamond$ is the standard LTL operator for reachability). With the implicit presence of the relinquishing action $\perp_{*}$, 
the situation can be modeled more naturally as in Figure 3 without having to add dummy sink locations and edges; or having to change the objective. Note that replicating the $a_{2}^{\prime}$ action to a similar player- 1 action from $l_{1}$ does not work in a $\perp_{*}$-less model, as we want the time at which the $a_{2}^{\prime}$ action is taken to be under the control of player 2 .

Clock regions. Timed automaton games can be solved using a region construction from the theory of timed automata AD94]. For a real $t \geq 0$, let $\operatorname{frac}(t)=t-\lfloor t\rfloor$ denote the fractional part of $t$. Given a timed automaton game $\mathcal{T}$, for each clock $x \in C$, let $c_{x}$ denote the largest integer constant that appears in any clock constraint involving $x$ in $\mathcal{T}$ (let $c_{x}=1$ if there is no clock constraint involving $x$ ). Two states $\left\langle l_{1}, \kappa_{1}\right\rangle$ and $\left\langle l_{1}, \kappa_{1}\right\rangle$ are said to be region equivalent if all the following conditions are satisfied: (a) $l_{1}=l_{2}$, (b) for all clocks $x, \kappa_{1}(x) \leq c_{x}$ iff $\kappa_{2}(x) \leq c_{x}$, (c) for all clocks $x$ with $\kappa_{1}(x) \leq c_{x},\left\lfloor\kappa_{1}(x)\right\rfloor=\left\lfloor\kappa_{2}(x)\right\rfloor$, (d) for all clocks $x, y$ with $\kappa_{1}(x) \leq c_{x}$ and $\kappa_{1}(y) \leq c_{y}$, frac $\left(\kappa_{1}(x)\right) \leq \operatorname{frac}\left(\kappa_{1}(y)\right)$ iff $\operatorname{frac}\left(\kappa_{2}(x)\right) \leq \operatorname{frac}\left(\kappa_{2}(y)\right)$, and (e) for all clocks $x$ with $\kappa_{1}(x) \leq c_{x}$, frac $\left(\kappa_{1}(x)\right)=0$ iff $\operatorname{frac}\left(\kappa_{2}(x)\right)=0$. A region is an equivalence class of states with respect to the region equivalence relation. There are finitely many clock regions; more precisely, the number of clock regions is bounded by $|L| \cdot \prod_{x \in C}\left(c_{x}+1\right) \cdot|C| ! \cdot 2^{2|C|}$.

Representing regions. A region of a timed automaton game $\mathcal{T}$ can be represented as a tuple $R=\langle l, h, \mathcal{P}(C)\rangle$ where (a) $l$ is a location of $\mathcal{T}$; (b) $h$ is a function which specifies the integral part of clocks $h: C \rightarrow(\mathbb{N} \cap[0, M])(M$ is the largest constant in $\mathcal{T})$; and (c) $\mathcal{P}(C)$ is a ordered disjoint partition of the clocks $\left\langle C_{-1}, C_{0}, \ldots C_{n}\right\rangle$ such that $\uplus C_{i}=C$, with $C_{i} \neq \emptyset$ for $i>0$. Then, a state $s$ with clock valuation $\kappa$ is in the region corresponding to $R$ when all the following conditions hold: (a) the location of $s$ corresponds to the location of $R$; (b) for all clocks $x$ with $\kappa(x) \leq c_{x},\lfloor\kappa(x)\rfloor=h(x)$; (c) for $\kappa(x)>c_{x}, h(x)=c_{x}$; (d) for all pair of clocks $(x, y)$, with $\kappa(x) \leq c_{x}$ and $\kappa(y) \leq c_{y}$, we have $\operatorname{frac}(\kappa(x))<\operatorname{frac}(\kappa(y))$ iff $x \in C_{i}$ and $y \in C_{j}$ with $0 \leq i<j$ (so, $x, y \in C_{k}$ with $k \geq 0$ implies $\operatorname{frac}(\kappa(x))=\operatorname{frac}(\kappa(y))$ ); (e) for $\kappa(x) \leq c_{x}$, frac $(\kappa(x))=0$ iff $x \in C_{0}$; and (f) $x \in C_{-1}$ iff $\kappa(x)>c_{x}$.

Region strategies and objectives. For a state $s \in S$, we write $\operatorname{Reg}(s) \subseteq S$ for the clock region containing $s$. For a run $r$, we let the region sequence $\operatorname{Reg}(r)=\operatorname{Reg}(r[0]), \operatorname{Reg}(r[1]), \cdots$. Two runs $r, r^{\prime}$ are region equivalent if their region sequences are the same. An $\omega$-regular objective $\Phi$ is a region objective if for all region-equivalent runs $r, r^{\prime}$, we have $r \in \Phi$ iff $r^{\prime} \in \Phi$. A strategy $\pi_{1}$ is a region strategy, if for all runs $r_{1}$ and $r_{2}$ and all $k \geq 0$ such that $\operatorname{Reg}\left(r_{1}[0 . . k]\right)=$ $\operatorname{Reg}\left(r_{2}[0 . . k]\right)$, we have that if $\pi_{1}\left(r_{1}[0 . . k]\right)=\left\langle\Delta, a_{1}\right\rangle$, then $\pi_{1}\left(r_{2}[0 . . k]\right)=\left\langle\Delta^{\prime}, a_{1}\right\rangle$ with $\operatorname{Reg}\left(r_{1}[k]+\Delta\right)=\operatorname{Reg}\left(r_{2}[k]+\Delta^{\prime}\right)$. The definition for player 2 strategies is analogous. Two region strategies $\pi_{1}$ and $\pi_{1}^{\prime}$ are region-equivalent if for all runs $r$ and all $k \geq 0$ we have that if $\pi_{1}(r[0 . . k])=\left\langle\Delta, a_{1}\right\rangle$, then $\pi_{1}^{\prime}(r[0 . . k])=\left\langle\Delta^{\prime}, a_{1}\right\rangle$ with $\operatorname{Reg}(r[k]+\Delta)=\operatorname{Reg}\left(r[k]+\Delta^{\prime}\right)$. A parity index function $\Omega$ is a region (resp. location) parity index function if $\Omega\left(s_{1}\right)=\Omega\left(s_{2}\right)$ whenever $\operatorname{Reg}\left(s_{1}\right)=\operatorname{Reg}\left(s_{2}\right)$ (resp. $s_{1}, s_{2}$ have the same location). Henceforth, we shall restrict our attention to region and location objectives.

Encoding time-divergence by enlarging the game structure. Given a timed automaton game $\mathcal{T}$, consider the enlarged game structure $\widehat{\mathcal{T}}$ (based mostly on the construction in $\left[\mathrm{dAFH}^{+} 03\right]$ ) with the state space $S^{\widehat{\mathcal{T}}} \subseteq S \times \mathbb{R}_{[0,1)} \times\{\text { TRUE, FALSE }\}^{2}$, and an augmented transition relation $\delta^{\widehat{\mathcal{T}}}: S^{\widehat{\mathcal{T}}} \times\left(M_{1} \cup M_{2}\right) \mapsto S^{\widehat{\mathcal{T}}}$. In an augmented state $\left\langle s, \mathfrak{z}, t i c k, b l_{1}\right\rangle \in S^{\widehat{\mathcal{T}}}$, the component $s \in S$ is a state of the original game structure $\llbracket \mathfrak{T} \rrbracket, \mathfrak{z}$ is the value of a fictitious clock $z$ which gets reset to 0 every time it hits 1 , tick is true iff $z$ hit 1 during the last transition, and $b l_{1}$ is true if player 1 is to blame for the last transition (i.e., blame $e_{1}$ is true 
for the last transition). Note that any strategy $\pi_{i}$ in $\llbracket \mathcal{T} \rrbracket$, can be considered a strategy in $\widehat{\mathcal{T}}$. The values of the clock $z$, tick and $b l_{1}$ correspond to the values each player keeps in memory in constructing his strategy. Given any initial value of $\mathfrak{z}=\mathfrak{z}^{*}, t i c k=t i c k^{*}, b l_{1}=b l_{1}^{*}$; any run $r$ in $\mathcal{T}$ has a corresponding unique run $\widehat{r}$ in $\widehat{\mathcal{T}}$ with $\widehat{r}[0]=\left\langle r[0], \mathfrak{z}^{*}, t i c k^{*}, b l_{1}^{*}\right\rangle$ such that $r$ is a projection of $\widehat{r}$ onto $\mathcal{T}$. For an objective $\Phi$, we can now encode time-divergence as the objective:

$$
\operatorname{TimeDivBl}_{1}(\Phi)=(\square \diamond t i c k \rightarrow \Phi) \wedge\left(\neg \square \diamond t i c k \rightarrow \diamond \square \neg b l_{1}\right)
$$

where $\square$ and $\diamond$ are the standard LTL modalities ("always" and "eventually" respectively), the combinations $\square \diamond$ and $\diamond \square$ denoting "infinitely often" and "all but for a finite number of steps" respectively. This is formalized in the following proposition.

Proposition 2.3. Let $\mathcal{T}$ be a timed automaton game and $\widehat{\mathcal{T}}$ be the corresponding enlarged game structure. Let $\Phi$ be an objective on $\mathcal{T}$. Consider a run $r=s^{0},\left\langle m_{1}^{0}, m_{2}^{0}\right\rangle, s^{1},\left\langle m_{1}^{1}, m_{2}^{1}\right\rangle, \ldots$ in $\mathcal{T}$. Let $\widehat{r}$ denote the corresponding run in $\widehat{\mathcal{T}}$ such that $\mathfrak{z}^{0}=0$, tick ${ }^{0}=$ FALSE, $b l_{1}^{0}=$ FALSE and $\widehat{r}=\left\langle s^{0}, \mathfrak{z}^{0}, t i c k^{0}, b l_{1}^{0}\right\rangle,\left\langle m_{1}^{0}, m_{2}^{0}\right\rangle,\left\langle s^{1}, \mathfrak{z}^{1}, t i c k^{1}, b l_{1}^{1}\right\rangle,\left\langle m_{1}^{1}, m_{2}^{1}\right\rangle, \ldots$ Then, $r \in \operatorname{TimeDivBl}_{1}(\Phi) \quad$ iff $\quad \widehat{r} \in\left((\square \diamond\right.$ tick $\rightarrow \Phi) \wedge\left(\neg \square \diamond\right.$ tick $\left.\left.\rightarrow \diamond \square \neg b l_{1}\right)\right)$.

Proof. Time diverges in the run $r$ iff it diverges in the corresponding run $\widehat{r}$. Moreover, time diverges in $\widehat{r}$ iff time crosses integer boundaries infinitely often, i.e., $\square \diamond$ tick holds. Also, the run $\widehat{r}$ belongs to Blameless 1 iff player 1 is blamed only finitely often, i.e., $\diamond \square \neg b l_{1}$ holds.

The following lemma states that because of the correspondence between $\mathcal{T}$ and $\widehat{\mathcal{T}}$, we can obtain the winning sets of $\mathcal{T}$ by obtaining the winning sets in $\widehat{\mathcal{T}}$.

Lemma 2.4. Let $\mathcal{T}$ be a timed automaton game and $\widehat{\mathcal{T}}$ be the corresponding enlarged game structure. Let $\Phi$ be an objective on $\mathcal{T}$. Any state $s$ of $\mathcal{T}$ satisfies $s \in \operatorname{Win}_{1}^{\mathcal{T}}\left(\operatorname{TimeDivBl}_{1}(\Phi)\right)$ iff $\langle s, 0$, FALSE, FALSE $\rangle \in \operatorname{Win}_{1}^{\widehat{\mathcal{T}}}\left((\square \diamond t i c k \rightarrow \Phi) \wedge\left(\neg \square \diamond t i c k \rightarrow \diamond \square \neg b l_{1}\right)\right)$.

Proof. Consider a state $s$ of $\mathcal{T}$, and a corresponding state $\langle s, 0$, FALSE, FALSE $\rangle$ of $\widehat{\mathcal{T}}$. The variables $\mathfrak{z}$, tick and $b l_{1}$ only "observe" properties in $\widehat{\mathcal{T}}$, they do not restrict transitions. Thus, given a run $r$ of $\mathcal{T}$ from $s$, there is a unique run $\widehat{r}$ of $\widehat{\mathcal{T}}$ from $\langle s, 0$, FALSE, FALSE $\rangle$ and vice versa. Similarly, any player- $i$ strategy $\pi_{i}$ in $\mathcal{T}$ corresponds to a strategy $\widehat{\pi}_{i}$ in $\widehat{\mathcal{T}}$; and any strategy $\widehat{\pi}_{i}$ in $\widehat{\mathcal{T}}$ corresponds to a strategy $\pi_{i}$ in $\mathcal{T}$ such that both strategies propose the same moves for corresponding runs. The result then follows from Proposition 2.3.

Encoding TimeDivBl$I_{1}(\operatorname{Parity}(\boldsymbol{\Omega}))$ as a parity objective. If $\Phi$ is a parity objective, then $\operatorname{TimeDivBl}_{1}(\Phi)$ can be specified as a parity objective in a related game structure $\widehat{\mathcal{T}}^{\Omega^{\text {td }}}$. The following encoding is based on a construction in $\mathrm{dAFH}^{+} 03$. Given $\Phi=\operatorname{Parity}(\Omega)$ where $\Omega$ is a parity index function of order $d$, the structure $\widehat{\mathcal{T}}^{\Omega^{\mathrm{td}}}$ has the state space $S^{\widehat{\widetilde{T}}^{\mathrm{td}}} \subseteq$ $S \times \mathbb{R}_{[0,1)} \times\{\text { TRUE, FALSE }\}^{2} \times\{0,1, \ldots, d-1\}$. Given a state $\widehat{s}=\left\langle s, \mathfrak{z}, t i c k, b l_{1}, p\right\rangle$, the set of available moves $\Gamma_{i}^{\widehat{\mathcal{T}}^{\mathrm{td}}}(\widehat{s})$ is equal to $\Gamma_{i}^{\mathcal{T}}(s)$. The transition relation $\delta^{\widehat{\mathcal{T}}^{\Omega^{\mathrm{td}}}}$ is specified as follows. For $\left\langle\Delta, a_{i}\right\rangle \in \Gamma_{i}^{\mathcal{T}}(s)$, we have $\delta^{\widehat{\widetilde{\tau}}^{\Omega^{\text {td }}}}\left(\left\langle s, \mathfrak{z}, t i c k, b l_{1}, p\right\rangle,\left\langle\Delta, a_{i}\right\rangle\right)=\left\langle s^{\prime}, \mathfrak{z}^{\prime}, t i c k^{\prime}, b l_{1}^{\prime}, p^{\prime}\right\rangle$ where

- $s^{\prime}=\delta^{\mathcal{T}}\left(s,\left\langle\Delta, a_{i}\right\rangle\right)$.

- $\mathfrak{z}^{\prime}=\operatorname{frac}(\mathfrak{z}+\Delta)$.

- tick $^{\prime}=$ TRUE iff $\mathfrak{z}+\Delta \geq 1$.

- $b l_{1}^{\prime}=$ TRUE iff $i=1$ (i.e., its a player-1 move). 
- $p^{\prime}= \begin{cases}\max \left(p, \Omega\left(s^{\prime}\right)\right) & \text { if tick }=\text { FALSE } \\ \Omega\left(s^{\prime}\right) & \text { if tick= TRUE }\end{cases}$

The following lemma states that a parity index function $\Omega^{\text {td }}$ can be defined on $\widehat{T}^{\Omega^{\text {td }}}$ such that the time divergence conditions are encoded. The proof of the lemma is technical and is presented in the appendix.

Lemma 2.5. Let $\mathcal{T}$ be a timed automaton game, Parity $(\Omega)$ an objective on $\mathcal{T}$, and $\widehat{\mathcal{T}}^{\Omega^{\mathrm{td}}}$ the corresponding enlarged game structure. Consider the parity index function $\Omega^{\mathrm{td}}$ for $\widehat{\mathcal{T}}^{\Omega^{\mathrm{td}}}$ defined as

$$
\Omega^{\mathrm{td}}\left(\left\langle s, \mathfrak{z}, t i c k, b l_{1}, p\right\rangle\right)= \begin{cases}0 & \text { if } t i c k=b l_{1}=\mathrm{FALSE} \\ 1 & \text { if } t i c k=\text { FALSE, } b l_{1}=\mathrm{TRUE} \\ p+2 & \text { if } t i c k=\mathrm{TRUE}\end{cases}
$$

Extend the parity function $\Omega$ to states of $\widehat{\mathcal{T}}^{\Omega^{\mathrm{td}}}$, such that the parity $\Omega(\langle s, \mathfrak{z}$, tick, bl,$p\rangle)$ is the same as the parity $\Omega(s)$ in $\mathcal{T}$. Then in the game structure $\widehat{\mathcal{T}}^{\Omega^{\mathrm{td}}}$, we have

$\operatorname{TimeDivBl}_{1}(\operatorname{Parity}(\Omega))=\left((\square \diamond\right.$ tick $\left.\rightarrow \operatorname{Parity}(\Omega)) \wedge\left(\neg \square \diamond t i c k \rightarrow \diamond \square \neg b l_{1}\right)\right)=\operatorname{Parity}\left(\Omega^{\text {td }}\right)$.

The next lemma states that we can consider games on $\widehat{\mathcal{T}}^{\Omega^{\text {td }}}$ with the parity index function $\Omega^{\text {td }}$ to obtain the winning states of $\mathcal{T}$ for the objective Parity $(\Omega)$. The proof of the lemma follows from the results of Lemma 2.4 (the result of the lemma also holds for the structure $\widehat{\mathcal{T}}^{\Omega^{\text {td }}}$ ) and Lemma 2.5 .

Lemma 2.6. Let $\mathcal{T}$ be a timed automaton game, $\Omega$ a parity index function on states of $\mathcal{T}, \widehat{\mathcal{T}}^{\Omega^{\mathrm{td}}}$ the corresponding enlarged game structure, and $\Omega^{\mathrm{td}}$ the parity index function on states of $\widehat{\mathcal{T}}^{\Omega^{\text {td }}}$ defined in Lemma 2.5. Let $\Omega$ be extended to states of $\widehat{\mathcal{T}}^{\Omega^{\text {td }}}$ such that $\Omega\left(\left\langle s, \mathfrak{z}\right.\right.$, tick $\left.\left., b l_{1}, p\right\rangle\right)=\Omega(s)$. Any state $s$ of $\mathcal{T}$ satisfies $s \in \operatorname{Win}_{1}^{\mathcal{T}}\left(\operatorname{TimeDivBl}_{1}(\operatorname{Parity}(\Omega))\right)$ iff $\langle s, 0$, FALSE, FALSE, 0$\rangle \in \mathrm{Win}_{1}^{\widehat{\widetilde{T}}^{\Omega^{\text {td }}}}\left((\square \diamond t i c k \rightarrow\right.$ Parity $\left.(\Omega)) \wedge\left(\neg \square \diamond t i c k \rightarrow \diamond \square \neg b l_{1}\right)\right)$ iff $\langle s, 0$, FALSE, FALSE, 0$\rangle \in \operatorname{Win}_{1}^{\widehat{\tau}^{\Omega^{\mathrm{td}}}}\left(\operatorname{Parity}\left(\Omega^{\mathrm{td}}\right)\right)$.

Let $\widehat{\kappa}$ be a valuation for the clocks in $\widehat{C}=C \cup\{z\}$. A state of $\widehat{\mathcal{T}}^{\Omega^{\text {td }}}$ can then be considered as $\left\langle\langle l, \widehat{\kappa}\rangle, t i c k, b l_{1}, p\right\rangle$. We extend the clock equivalence relation to these expanded states: $\left\langle\langle l, \widehat{\kappa}\rangle, t i c k, b l_{1}, p\right\rangle \cong\left\langle\left\langle l^{\prime}, \widehat{\kappa}^{\prime}\right\rangle, t i c k^{\prime}, b l_{1}^{\prime}, p^{\prime}\right\rangle$ iff $l=l^{\prime}, t i c k=t i c k^{\prime}, b l_{1}=b l_{1}^{\prime}, p=p^{\prime}$ and $\widehat{\kappa} \cong \widehat{\kappa}^{\prime}$. We let $\left\langle l, t i c k, b l_{1}, p\right\rangle$ be the "locations" in $\widehat{\mathcal{T}}^{\Omega^{\mathrm{td}}}$. If $\Omega$ is a location parity index function for $\mathcal{T}$, we have $\Omega^{\text {td }}$ to be a location parity index function for $\widehat{\mathcal{T}}^{\Omega^{\mathrm{td}}}$

A $\mu$-calculus formulation for describing the winning set. A $\mu$-calculus formula $\varphi$ to describe the winning set $\operatorname{Win}_{1}^{\widehat{\tau}^{\Omega^{\text {td }}}}\left(\operatorname{Parity}\left(\Omega^{\text {td }}\right)\right)$ is given in $\left[\mathrm{dAFH}^{+} 03\right.$. The $\mu$-calculus formula uses the controllable predecessor operator for player 1 , CPre $1: 2^{\widehat{S}} \mapsto 2^{\widehat{S}}$ (where $\widehat{S}=S^{\widehat{\tau}^{\Omega^{\mathrm{td}}}}$ ), defined formally by

$$
\widehat{s} \in \operatorname{CPre}_{1}(Z) \quad \text { iff } \quad \exists m_{1} \in \Gamma_{1}^{\widehat{\widetilde{\tau}}^{\Omega^{\mathrm{td}}}}(\widehat{s}) \forall m_{2} \in \Gamma_{2}^{\widehat{\mathcal{T}}^{\Omega^{\mathrm{td}}}}(\widehat{s}) \cdot \delta_{\mathrm{jd}}^{\widehat{\mathrm{j}}^{\Omega^{\mathrm{td}}}}\left(\widehat{s}, m_{1}, m_{2}\right) \subseteq Z .
$$

Informally, $\mathrm{CPre}_{1}(Z)$ consists of the set of states from which player 1 can ensure that the next state will be in $Z$, no matter what player 2 does. The operator $\mathrm{CPre}_{1}$ preserves regions of $\widehat{\mathcal{T}}^{\Omega^{\text {td }}}$ (this follows from the results of Lemma 3.1). It follows from $\mathrm{dAFH}{ }^{+} 03$ that given 
a parity index function $\widehat{\Omega}: \widehat{S} \mapsto\{0,1, \ldots, d-1\}$, the winning set $\operatorname{Win}_{1}^{\widehat{\tau}^{\text {td }}}(\operatorname{Parity}(\widehat{\Omega}))$ can be described by the following $\mu$-calculus formula in case $d-1$ is odd:

$$
\mu X_{d-1} \cdot \nu Y_{d-2} \cdot \mu X_{d-3} \ldots \nu Y_{2} \cdot \mu X_{1} \cdot \nu Y_{0}\left[\begin{array}{rl}
\widehat{\Omega}^{-1}(0) & \cap \mathrm{CPre}_{1}\left(Y_{0}\right) \\
& \cup \\
\widehat{\Omega}^{-1}(1) & \cap \operatorname{CPre}_{1}\left(X_{1}\right) \\
& \cup \\
\widehat{\Omega}^{-1}(2) & \cap \mathrm{CPre}_{1}\left(Y_{2}\right) \\
\vdots & \\
\widehat{\Omega}^{-1}(d-2) & \cap \operatorname{CPre}_{1}\left(Y_{d-2}\right) \\
& \cup \\
\widehat{\Omega}^{-1}(d-1) & \cap \operatorname{CPre}_{1}\left(X_{d-1}\right)
\end{array}\right]
$$

where $\mu$ and $\nu$ denote the least fixpoint and the greatest fixpoint operators respectively, and $\widehat{\Omega}^{-1}(j)$ denotes the set of states of parity $j$. In case $d-1$ is even, the $\mu$-calculus formula is

$$
\nu Y_{d-1} \cdot \mu X_{d-2} \cdot \nu X_{d-3} \ldots \mu Y_{2} \cdot \nu X_{1} \cdot \mu Y_{0}\left[\begin{array}{rl}
\widehat{\Omega}^{-1}(0) & \cap \mathrm{CPre}_{1}\left(Y_{0}\right) \\
& \cup \\
\widehat{\Omega}^{-1}(1) & \cap \operatorname{CPre}_{1}\left(X_{1}\right) \\
& \cup \\
\widehat{\Omega}^{-1}(2) & \cap \operatorname{CPre}_{1}\left(Y_{2}\right) \\
\vdots \\
\widehat{\Omega}^{-1}(d-2) & \cap \operatorname{CPre}_{1}\left(X_{d-2}\right) \\
\cup & \\
\widehat{\Omega}^{-1}(d-1) & \cap \operatorname{CPre}_{1}\left(Y_{d-1}\right)
\end{array}\right]
$$

We now present a lemma which states that in the structure $\widehat{\mathcal{T}}^{\Omega^{\text {td }}}$, for location $\omega$-regular objectives, (1) memoryless region strategies suffice for winning, and (2) from states in the winning set there exists a winning memoryless region strategy $\pi_{1}$ such that all strategies region-equivalent to $\pi_{1}$ are also winning. The proof of the lemma can be found in the appendix.

Lemma 2.7. Let $\mathcal{T}$ be a timed automaton game, $\Omega$ a location parity index function on states of $\mathcal{T}$, and $\widehat{\mathcal{T}}^{\Omega^{\text {td }}}$ the corresponding enlarged game structure with the parity index function $\Omega^{\mathrm{td}}$. Then, (1) there exists a memoryless region winning strategy $\pi_{1}$ for Parity $\left(\Omega^{\mathrm{td}}\right)$ from $\mathrm{Win}_{1}^{\widehat{\widetilde{\Omega}}^{\mathrm{td}}}\left(\operatorname{Parity}\left(\Omega^{\mathrm{td}}\right)\right)$, and (2) if $\pi_{1}^{\prime}$ is a strategy that is region-equivalent to $\pi_{1}$, then $\pi_{1}^{\prime}$ is a winning strategy for $\operatorname{Parity}\left(\Omega^{\mathrm{td}}\right)$ from $\mathrm{Win}_{1}^{\widehat{\widetilde{T}}^{\mathrm{td}}}\left(\operatorname{Parity}\left(\Omega^{\mathrm{td}}\right)\right)$.

We say a strategy $\pi_{i}$ is move-independent if for any two runs $r, r^{\prime}$ such that $r[k]=r^{\prime}[k]$ for all $k \geq 0$ we have $\pi_{i}(r[0 . . j])=\pi\left(r^{\prime}[0 . . j]\right)$ for all $j \geq 0$. A move-independent region strategy is a region strategy that is move-independent. The following corollary follows from Lemma 2.7 observing that a memoryless strategy is a move independent strategy. A memoryless strategy in $\widehat{\mathcal{T}}^{\Omega^{\text {td }}}$ does not always have a corresponding memoryless strategy in $\mathcal{T}$. It may not even have a move-independent strategy in $\mathcal{T}$. This is because to infer the values of $\mathfrak{z}, t i c k, b l_{1}$ in $\mathcal{T}$, we need the values of the previous moves taken. The following proposition states that if player 1 has access to a global clock then move-independent strategies suffice in $\mathcal{T}$ (the proof can be found in the appendix). 
Proposition 2.8. Let $\mathcal{T}$ be a timed automaton game such that the set of clocks includes a global clock $z$ that is never reset and let $\Phi=\operatorname{Parity}(\Omega)$ be an $\omega$-regular location objective of $\mathcal{T}$. Then, move-independent strategies in $\mathcal{T}$ suffice for winning $\operatorname{TimeDivBl}_{1}(\Phi)$.

The next lemma states that memoryless strategies of player 2 suffice as spoiling strategies (the proof is in the appendix).

Lemma 2.9. Let $\mathcal{T}$ be a timed automaton game, $\Omega$ a location parity index function on states of $\mathcal{T}$, and $\widehat{\mathcal{T}}^{\Omega^{\mathrm{td}}}$ the corresponding enlarged game structure with the parity index function $\Omega^{\mathrm{td}}$. Then, memoryless region strategies of player 2 in $\widehat{\mathcal{T}}^{\Omega^{\text {td }}}$ suffice for preventing player 1 from winning $\operatorname{Parity}\left(\Omega^{\mathrm{td}}\right)$ from a state $\widehat{s} \notin \mathrm{Win}_{1}^{\widehat{\tau}^{\Omega^{\mathrm{td}}}}\left(\operatorname{Parity}\left(\Omega^{\mathrm{td}}\right)\right)$.

\section{Exact Winning of Timed Parity Games}

In this section we will present a reduction of infinite-state timed automaton games with parity objectives to finite-state turn-based games with parity objectives. The reduction gives us several results related to complexity and algorithms to solve timed automata parity games: (a) we obtain algorithms to solve timed automaton parity games with better time complexity than the algorithm presented in $\left[\mathrm{dAFH}^{+} 03\right]$; (b) our reduction allows us to use the rich literature on algorithms for finite-state parity games for solving timed automaton parity games.

Finite-state turn-based games. A finite-state turn-based game $G$ consists of the tuple $\left\langle(S, E),\left(S_{1}, S_{2}\right)\right\rangle$, where $\left(S_{1}, S_{2}\right)$ forms a partition of the finite set $S$ of states, $E$ is the set of edges, $S_{1}$ is the set of states from which only player 1 can make a move to choose an outgoing edge, and $S_{2}$ is the set of states from which only player 2 can make a move. The game is bipartite if every outgoing edge from a player- 1 state leads to a player- 2 state and vice-versa.

First idea of the reduction. Let $\mathcal{T}$ be a timed automaton game, and let $\widehat{T}^{\Omega^{\text {td }}}$ be the corresponding enlarged timed game structure that encodes time divergence. We shall construct a finite-state turn-based game structure $\mathcal{T}^{f}$ based on the regions of $\widehat{\mathcal{T}}^{\Omega^{\text {td }}}$ which can be used to compute winning states for parity objectives for the timed automaton game $\mathcal{T}$. In this finite-state game, first player 1 proposes a destination region $\widehat{R}_{1}$ together with a discrete action $a_{1}$ (intuitively, this can be taken to mean that in the game $\widehat{\mathcal{T}}^{\Omega^{\mathrm{td}}}$, player 1 wants to first let time elapse to get to the region $\widehat{R}_{1}$, and then take the discrete action $a_{1}$ ). The finite-state game then moves to an intermediate state which remembers the proposed action of player 1 in the game $\widehat{\mathcal{T}}^{\Omega^{\text {td }}}$. Let us denote this intermediate state in $\mathcal{T}^{f}$ which specifies the desired destination region and action of player 1 in $\widehat{\mathcal{T}}^{\Omega^{\text {td }}}$ by the tuple $\left\langle\widehat{R}, \widehat{R}_{1}, a_{1}\right\rangle$. From this state in $\mathcal{T}^{f}$, player 2 similarly also proposes a move consisting of a region $\widehat{R}_{2}$ together with a discrete action $a_{2}$. These two moves in $\mathcal{T}^{f}$ signify that in the game in $\widehat{\mathcal{T}}^{\Omega^{\mathrm{td}}}$ player $i$ proposed a move $\left\langle\Delta_{i}, a_{i}\right\rangle$ from a state $\widehat{s} \in \widehat{R}$ such that $\widehat{s}+\Delta_{i} \in \widehat{R}_{i}$. Depending on the move $\left\langle\Delta_{2}, a_{2}\right\rangle$, the game in $\mathcal{T}^{f}$ will then proceed from $\left\langle\widehat{R}, \widehat{R}_{1}, a_{1}\right\rangle$ to destination states $\operatorname{Reg}\left(\delta^{\widehat{\tau}^{\Omega^{\mathrm{td}}}}\left(\widehat{s},\left\langle\Delta_{1}, a_{1}\right\rangle\right)\right)$, or $\operatorname{Reg}\left(\delta^{\widehat{\mathcal{T}}^{\Omega^{\mathrm{td}}}}\left(\widehat{s},\left\langle\Delta_{2}, a_{2}\right\rangle\right)\right)$, or both, after the move of player 2 depending on whether $\Delta_{1}<\Delta_{2}$, or, $\Delta_{1}>\Delta_{2}$, or, $\Delta_{1}=\Delta_{2}$, respectively. The following lemma indicates that only the regions of $\widehat{s}+\Delta_{i}$ are important in determining whether the 
move of player 1 or player 2 determines the successors after this two-step process. The proof is technical and is presented in the appendix.

Lemma 3.1. Let $\mathcal{T}$ be a timed automaton game, $\Omega$ a parity index function on states of $\mathcal{T}$ and let $Y, Y_{1}^{\prime}, Y_{2}^{\prime}$ be regions in the enlarged timed game structure $\widehat{\mathcal{T}}^{\Omega^{\mathrm{td}}}$. Suppose player-i has a move $\left\langle\Delta_{i}, \perp_{i}\right\rangle$ from some $\widehat{s} \in Y$ to $\widehat{s}_{i} \in Y_{i}^{\prime}$, for $i \in\{1,2\}$. Then, for all states $\widehat{s} \in Y$ and for all player-1 moves $m_{1}^{\widehat{s}}=\left\langle\Delta_{1}, a_{1}\right\rangle$ with $\widehat{s}+\Delta_{1} \in Y_{1}^{\prime}$ and $a_{1} \neq \perp_{*}$, one of the following cases must hold.

(1) $Y_{1}^{\prime} \neq Y_{2}^{\prime}$ and for all moves $m_{2}^{\widehat{s}}=\left\langle\Delta_{2}, a_{2}\right\rangle$ of player-2 with $\widehat{s}+\Delta_{2} \in Y_{2}^{\prime}$, we have $\Delta_{1}<$ $\Delta_{2}\left(\right.$ and hence blame $1\left(\widehat{s}, m_{1}^{\widehat{s}}, m_{2}^{\widehat{s}}, \widehat{\delta}\left(\widehat{s}, m_{1}^{\widehat{s}}\right)\right)=$ TRUE and blame $2\left(\widehat{s}, m_{1}^{\widehat{s}}, m_{2}^{\widehat{s}}, \widehat{\delta}\left(\widehat{s}, m_{2}^{\widehat{s}}\right)\right)=$ FALSE).

(2) $Y_{1}^{\prime} \neq Y_{2}^{\prime}$ and for all player-2 moves $m_{2}^{\widehat{s}}=\left\langle\Delta_{2}, a_{2}\right\rangle$ with $\widehat{s}+\Delta_{2} \in Y_{2}^{\prime}$, we have $\Delta_{2}<$ $\Delta_{1}\left(\right.$ and hence blame $2\left(\widehat{s}, m_{1}^{\widehat{s}}, m_{2}^{\widehat{s}}, \widehat{\delta}\left(\widehat{s}, m_{2}^{\widehat{s}}\right)\right)=$ TRUE and blame $1\left(\widehat{s}, m_{1}^{\widehat{s}}, m_{2}^{\widehat{s}}, \widehat{\delta}\left(\widehat{s}, m_{1}^{\widehat{s}}\right)\right)=$ FALSE).

(3) $Y_{1}^{\prime}=Y_{2}^{\prime}$ and there exists a player 2 move $m_{2}^{\widehat{s}}=\left\langle\Delta_{2}, a_{2}\right\rangle$ with $\widehat{s}+\Delta_{2} \in Y_{2}^{\prime}$ such that $\Delta_{1}=$ $\Delta_{2}\left(\right.$ and hence blame $1\left(\widehat{s}, m_{1}^{\widehat{s}}, m_{2}^{\widehat{s}}, \widehat{\delta}\left(\widehat{s}, m_{1}^{\widehat{s}}\right)\right)=$ TRUE and blame $2\left(\widehat{s}, m_{1}^{\widehat{s}}, m_{2}^{\widehat{s}}, \widehat{\delta}\left(\widehat{s}, m_{2}^{\widehat{s}}\right)\right)=$ TRUE).

Lemma 3.1 states that given an initial state in $\widehat{R}$, for moves of both players to some fixed $\widehat{R}_{1}, \widehat{R}_{2}$, either the move of player 1 is always chosen, or player 2 can always pick a move such that player-1's move is foiled.

Let $S_{\text {Reg }}^{\widehat{\widetilde{\tau}}^{\Omega^{\mathrm{td}}}}=\left\{X \mid X\right.$ is a region of $\left.\widehat{\mathcal{T}}^{\Omega^{\text {td }}}\right\}$, and let $S^{\dagger}=S_{\mathrm{Reg}}^{\widehat{\widetilde{\tau}}^{\mathrm{td}}} \times S_{\mathrm{Reg}}^{\widehat{\widetilde{\Omega}}^{\mathrm{td}}} \times A_{1}^{\perp}$. Using Lemma 3.1. a bipartite turn-based finite game $\mathcal{T}^{f}=\left\langle\left(S^{f}, E^{f}\right),\left(S_{\operatorname{Reg}}^{\widehat{\mathrm{T}}^{\mathrm{td}}} \times\{1\}, S^{\dagger} \times\{2\}\right)\right\rangle$ can be constructed to capture the timed game $\mathcal{T}$ as follows.

- The state space $S^{f}$ is equal to $S_{\text {Reg }}^{\widehat{\mathrm{T}}^{\Omega^{\text {td }}}} \times\{1\} \cup S^{\dagger} \times\{2\}$.

- Each $\langle\widehat{R}, 1\rangle \in S_{\mathrm{Reg}}^{\widehat{\mathrm{T}}^{\Omega^{\mathrm{td}}}} \times\{1\}$ encodes states in the timed game $\widehat{\mathcal{T}}^{\Omega^{\mathrm{td}}}$ that belongs to the region $\widehat{R} \cdot S_{\mathrm{Reg}}^{\widehat{\widetilde{\tau}}^{\mathrm{td}}} \times\{1\}$ are player-1 states.

- Each $\langle Y, 2\rangle \in S^{\dagger} \times\{2\}$ encodes the following information: (a) the previous state of $\mathcal{T}^{f}$ (which corresponds to a region $\widehat{R}$ of $\widehat{\mathcal{T}}^{\Omega^{\mathrm{td}}}$ ), (b) a region $\widehat{R}^{\prime}$ of $\widehat{\mathcal{T}}^{\Omega^{\mathrm{td}}}$ (representing an intermediate state which results from time passage in $\widehat{\mathcal{T}}^{\Omega^{\text {td }}}$ from the state in the previous region $\widehat{R}$ to a state in $\widehat{R}^{\prime}$ ), and (c) the desired discrete action of player 1 to be taken from the intermediate state in $\widehat{R}^{\prime} . S^{\dagger} \times\{2\}$ are player-2 states.

- An edge from $\langle\widehat{R}, 1\rangle$ to $\langle Y, 2\rangle=\left\langle\widehat{R}, \widehat{R}^{\prime}, a_{1}, 2\right\rangle$ would represent the fact that in the timed game $\widehat{\mathcal{T}}^{\Omega^{\mathrm{td}}}$, from some state $\widehat{s} \in \widehat{R}$, player 1 has a move $\left\langle\Delta, a_{1}\right\rangle$ such that $\widehat{s}+\Delta$ is in the intermediate region component $\widehat{R}^{\prime}$ of $\langle Y, 2\rangle$, with $a_{1}$ being the desired final discrete action. From the state $\langle Y, 2\rangle$, player 2 would have moves to $S_{\operatorname{Reg}}^{\widehat{\widetilde{\tau}}^{\text {td }}} \times\{1\}$ depending on what moves of player 2 in the timed game $\widehat{\mathcal{T}}^{\Omega^{\mathrm{td}}}$ can beat the player-1 moves from $\widehat{R}$ to $\widehat{R}^{\prime}$ according to Lemma 3.1

The construction alluded to above requires having a state space that has size roughly $\left|S_{\mathrm{Reg}}^{\widehat{\widetilde{T}}^{\Omega^{\mathrm{td}}}}\right|^{2} \cdot\left|A_{1}\right|$, where $\left|S_{\mathrm{Reg}}^{\widehat{\mathrm{T}}^{\mathrm{td}}}\right|$ is the number of regions of $\widehat{\mathcal{T}}^{\Omega^{\mathrm{td}}}$. An optimized construction was presented in [CHP08] such that the size of the state space was roughly $O\left(\left|S_{\operatorname{Reg}}^{\widehat{\widetilde{\Omega}}^{\mathrm{td}}}\right| \cdot M \cdot|C| \cdot\left|A_{1}\right|\right)$ where $M$ is the largest constant and $C$ the set of clocks in $\widehat{A}^{\Omega^{\mathrm{td}}}$. The optimization was due 
to the observation that a state in a region $\widehat{R}$ does not have transitions to every region, but only to a few restricted ones.

Second idea of the reduction. We will show in subsection 3.2 that the strategies of both players in the timed game $\widehat{\mathcal{T}}^{\Omega^{\text {td }}}$ can be be restricted so that from any state $\widehat{s}$, each player can only propose moves $\left\langle\Delta_{i}, a_{i}\right\rangle$ such that the discrete action $a_{i}$ is taken either from the current region, or from the two following successor regions. That is, the cardinality of the set $\left\{\operatorname{Reg}(\widehat{s}+\Delta) \mid 0 \leq \Delta \leq \Delta_{i}\right\}$ is at most 3. The winning set remains the set with strategies being restricted in this manner. This result allows us to restrict the size of the state space in the region pair construction mentioned above to $O\left(\left|S_{\operatorname{Reg}}^{\widehat{\widetilde{T}}^{\mathrm{td}}}\right| \cdot\left|A_{1}\right|\right)$. A further optimization allows us to have a state space linear in $\left|S_{\mathrm{Reg}}^{\widehat{\tau}^{\text {td }}}\right|$.

Outline of Section 3. In subsection 3.1, we show that the strategies of both players can be restricted so that the edges of the timed automaton game are taken from the current region, or from the two following successor regions; with the restriction not changing the winning set $\mathrm{Win}_{1}^{\widehat{\widetilde{T}}^{\Omega^{\mathrm{td}}}}\left(\operatorname{Parity}\left(\Omega^{\mathrm{td}}\right)\right)$. We call these restricted games 3-region timed parity games. Then in subsection [3.2, we reduce these 3-region timed parity games to finite-state turn-based games, the state space of the turn-based game being linear in the number of regions of $\widehat{\mathcal{T}}^{\Omega^{\text {td }}}$.

\subsection{Reduction to 3-Region Timed Parity Games.}

3.1.1. 3-Region strategies. We define the boolean functions Succr ${\widehat{\Upsilon^{\Omega}}}^{\mathrm{td}^{\mathrm{d}}}: S^{\widehat{\widetilde{\Upsilon}}^{\Omega^{\mathrm{td}}}} \times \mathbb{R}_{\geq 0} \mapsto$ \{TRUE, FALSE $\}$ for $j \in\{2,3\}$ as

$$
\operatorname{Succr}_{j}^{\widehat{\top}^{\Omega^{\mathrm{td}}}}(\widehat{s}, \Delta)= \begin{cases}\text { TRUE } & \text { if }\left|\left\{\operatorname{Reg}\left(\widehat{s}+\Delta^{\prime}\right) \mid 0 \leq \Delta^{\prime} \leq \Delta\right\}\right| \leq j \\ \text { FALSE } & \text { otherwise }\end{cases}
$$

For player $i$, with $i \in\{1,2\}$, we say a strategy $\pi_{i}$ is a 3-region strategy of $\widehat{\mathcal{T}}^{\Omega^{\text {td }}}$ if for any run prefix $r[0 . . k]$, we have that $\pi_{i}$ plays a move of duration $\Delta$ such that $r[k]+\Delta$ is at most in the second following region. Formally, $\pi_{i}(r[0 . . k])=\left\langle\Delta_{i}^{k}, a_{i}^{k}\right\rangle$ with $\operatorname{Succr}_{3}^{\Omega^{\text {td }}}\left(r[k], \Delta_{i}^{k}\right)=$ TRUE. We wish to show that in the game $\widehat{\mathcal{T}}^{\Omega^{\text {td }}}$, we can restrict both players to using only 3-region strategies. Consider 3-region strategies of player 1. Informally, they suffice for winning as a) 3-region strategies allow time to diverge, and b) if $\pi_{1}$ is a player-1 winning strategy, then we can obtain a winning 3-region strategy $\pi_{1}^{*}$ that plays the same moves as $\pi_{1}$ whenever $\pi_{1}$ proposes moves within two successor regions; and plays simple time moves to the second successor region whenever $\pi_{1}$ plays a move to outside the second following region. The strategy $\pi_{1}^{*}$ works in general because for a run $\widehat{r}[0 . . k]$ such that $\pi_{1}$ proposes moves outside two successor regions, a player-2 strategy $\pi_{2}$ can counter $\pi_{1}$ by playing similar pure time move as $\pi_{1}^{*}$. Unfortunately this argument does not formally work, as a player- 1 move makes the $b l_{1}$ component true, and a player-2 move makes $b l_{1}$ false, that is, $\delta\left(r[k],\left\langle\Delta, \perp_{1}\right\rangle\right) \neq \delta\left(r[k],\left\langle\Delta, \perp_{2}\right\rangle\right)$, the only difference being in the $b l_{1}$ components. We get around this roadblock by working in another expanded game structure where the $b l_{1}$ component is true only if a move of player 1 is chosen, and the move is either to the originating region, or to the immediately succeeding region. It turns out that this modification does not change the time divergence condition. We present this new game structure next. 
3.1.2. The expanded game structure $\widehat{\mathcal{T}}_{3 b l}^{\Omega^{\mathrm{td}}}$. Analogous to the definition of $\widehat{\mathcal{T}}^{\Omega^{\mathrm{td}}}$, let $\widehat{\mathcal{T}}_{3 b l}^{\Omega^{\mathrm{td}}}$ be a similar enlarged game structure, the only difference from $\widehat{\mathcal{T}}^{\Omega^{\text {td }}}$ being in the $b l_{1}$ component (we will refer to the new $b l_{1}$ component in the new game structure as $3 b l$ ). We denote the transition relation by $\delta^{\widehat{\mathcal{T}}_{3 b l}^{\text {td }}}$, and the joint transition relation by $\delta_{\text {jd }}^{\widehat{\widetilde{T}}_{3 b l}^{\Omega^{\text {td }}}}$. In an augmented state $\left\langle s, \mathfrak{z}, t i c k, 3 b l_{1}, p\right\rangle \in S^{\widehat{\widetilde{T}}_{3 b l}^{\text {td }}}$, the component $3 b l_{1}$ is true only if both of the following conditions are satisfied:

(a) player 1 is to blame for the last transition, and

(b) if $\left.\left.\quad \delta^{\widehat{\widetilde{\tau}}_{3 b l}^{\Omega^{\text {td }}}} \underset{\widehat{\widetilde{\tau}}^{\Omega^{\mathrm{td}}}}{\left(\left\langle s^{*}\right.\right.}, \mathfrak{z}^{*}, t i c k^{*}, 3 b l_{1}^{*}, p^{*}\right\rangle,\left\langle\Delta, a_{1}\right\rangle\right)=\left\langle s, \mathfrak{z}, t i c k, 3 b l_{1}, p\right\rangle$

then $\operatorname{Succr}_{2}^{\widehat{T}_{3 b l}^{\text {td }}}\left(\left\langle s^{*}, \mathfrak{z}^{*}, t i c k^{*}, 3 b l_{1}^{*}, p\right\rangle, \Delta\right)=$ TRUE.

A run $r$ of $\mathcal{T}$ has corresponding unique run $\widehat{r}$ in $\widehat{\mathcal{T}}^{\Omega^{\mathrm{td}}}$ and $\widehat{r}_{3}$ in $\widehat{\mathcal{T}}_{3 b l}^{\Omega^{\mathrm{td}}}$ such that $r$ is a projection of $\widehat{r}$ and $\widehat{r}_{3}$ onto $\mathcal{T}$, given the starting values of $\mathfrak{z}$, tick, $b l_{1}, 3 b l_{1}$ and $p$. The reverse also holds - for any run in the expanded game structures starting from $\left\langle s, \mathfrak{z}, t i c k, b l_{1}, p\right\rangle$, we have a corresponding unique run in $\mathcal{T}$ from $s$. Observe that the available moves are the same in all game structures, and we may view the additional components of the states in the expanded game structures as being kept in memory by the two players in $\mathcal{T}$. A similar correspondence between strategies also holds, thus a strategy $\pi_{i}$ in $\mathcal{T}$ has corresponding matching strategies in $\widehat{\mathcal{T}}^{\Omega^{\mathrm{td}}}$ and in $\widehat{\mathcal{T}}_{3 b l}^{\Omega^{\mathrm{td}}}$; and vice versa.

The next lemma states that time diverges in a run $\widehat{r}$ of $\widehat{\mathcal{T}}_{3 b l}^{\text {td }}$ when the run has infinitely many winning moves such that the moves allow a time elapse to a region farther than the immediate successor region.

Lemma 3.2. Consider a run $r \in \mathcal{T}$ such that $r=s_{0},\left\langle m_{1}^{0}, m_{2}^{0}\right\rangle, s_{1},\left\langle m_{1}^{1}, m_{2}^{1}\right\rangle, \ldots$ and the corresponding runs $\widehat{r}$ and $\widehat{r}_{3}$ in $\widehat{\mathcal{T}}^{\Omega^{\mathrm{td}}}$ and $\widehat{\mathcal{T}}_{3 b l}^{\text {td }}$ respectively. Suppose for infinitely many $k$ we have Succr $\widehat{\widetilde{T}}^{\Omega^{\mathrm{td}}}\left(\widehat{r}[k]\right.$, delay $\left.\left(m_{1}^{k}, m_{2}^{k}\right)\right)$ to be FALSE (or $\operatorname{Succr}_{2} \widehat{\mathrm{T}}^{\Omega^{\mathrm{td}}}\left(\widehat{r}_{3}[k]\right.$, delay $\left.\left(m_{1}^{k}, m_{2}^{k}\right)\right)$ to be FALSE). Then time diverges in the run $r$ (and hence also in $\widehat{r}, \widehat{r}_{3}$ ).

Proof. We have that for all $k \geq 0$, except for the blame component, the remaining four corresponding components of the 5-tuples $\widehat{r}[k]$ and of $\widehat{r}_{3}[k]$ match. Thus,

$$
\operatorname{Succr}_{2}^{\widehat{\Im}^{\Omega^{\mathrm{td}}}}\left(\widehat{r}[k], \text { delay }\left(m_{1}^{k}, m_{2}^{k}\right)\right)=\operatorname{Succr}_{2}^{\widehat{\tau}^{\Omega^{\mathrm{td}}}}\left(\widehat{r}_{3}[k] \text {, delay }\left(m_{1}^{k}, m_{2}^{k}\right)\right) \text {. }
$$

A region of $\widehat{\mathcal{T}}^{\Omega^{\text {td }}}$ can be represented as a tuple $\widehat{R}=\left\langle l, t i c k, b l_{1}, p, \widehat{h}, \mathcal{P}(\widehat{C})\right\rangle$ (similar to clock regions in $\mathcal{T}$ ) where (a) $h$ is a function which specifies the integer values of clocks $h: \widehat{C} \rightarrow(\mathbb{N} \cap[0, M])(M$ is the largest constant in $\mathcal{T})$; and $(\mathrm{b}) \mathcal{P}(\widehat{C})$ is a disjoint partition of the clocks $\left\langle\widehat{C}_{-1}, \widehat{C}_{0}, \ldots \widehat{C}_{n}\right\rangle$ such that $\uplus \widehat{C}_{i}=\widehat{C}$, and $\widehat{\widehat{C}}_{i} \neq \emptyset$ for $i>0$ (see Section 2 for details on regions and this representation).

Consider the clock partitions $\mathcal{P}^{k}(\widehat{C})=\left\langle\widehat{C}_{-1}^{k}, \widehat{C}_{0}^{k}, \ldots \widehat{C}_{n^{k}}^{k}\right\rangle$ of the regions $\operatorname{Reg}(\widehat{r}[k])$. Suppose $\widehat{C}_{-1}^{k} \neq \emptyset$. Then, the immediate time successor of the region will have $\widehat{C}_{-1}=\emptyset$, and $\widehat{C}_{j}=\widehat{C}_{j-1}^{k}$ for $n^{k}+1 \geq j \geq 0$. Suppose $\widehat{C}_{-1}^{k}=\emptyset$, then the immediate time successor of the region will have $\widehat{C}_{-1} \neq \emptyset$; this happens because all the clock values in $\widehat{C}_{n^{k}}^{k}$ reach an integer boundary. Suppose $\operatorname{Succr}_{2}^{\widehat{\Upsilon}^{\mathrm{td}}}\left(\widehat{r}[k]\right.$, delay $\left.\left(m_{1}^{k}, m_{2}^{k}\right)\right)=$ FALSE. This means that $\operatorname{Reg}\left(\widehat{r}[k]+\operatorname{delay}\left(m_{1}^{k}, m_{2}^{k}\right)\right)$ is at least two region successors away from $\operatorname{Reg}(\widehat{r}[k])$. Thus, some clock must be crossing an integer boundary (greater than 0$)$ between time $(\widehat{r}, k)$ and 
$\operatorname{time}(\widehat{r}, k+1)$. Since $\operatorname{Succr}_{2}^{\widehat{\tau}^{\Omega^{\mathrm{td}}}}\left(\widehat{r}[k]\right.$, delay $\left.\left(m_{1}^{k}, m_{2}^{k}\right)\right)=$ FALSE for infinitely many $k$, we must have that some clock crosses an integer boundary greater than 1 infinitely often. Observing that at least one time unit must pass between any two such crossings, we have that time diverges in the run $\widehat{r}$ (and hence also in $r, \widehat{r}_{3}$ ).

We next show that the change in the $b l_{1}$ component in $\widehat{\mathcal{T}}_{3 b l}^{\Omega \text { td }}$ does not change the time divergence condition. Given an objective $\Phi$, let $\operatorname{TimeDiv}_{3 \mathrm{BI}_{1}}(\Phi)$ denote the objective $(\square \diamond t i c k \rightarrow \Phi) \wedge\left(\neg \square \diamond t i c k \rightarrow \diamond \square \neg 3 b l_{1}\right)$.

Lemma 3.3. Let $\mathcal{T}$ be a timed automaton game, $\Omega$ a location parity index function on states of $\mathcal{T}$, and $\widehat{\mathcal{T}}^{\Omega^{\mathrm{td}}}$ the corresponding enlarged game. Consider a run $r \in \mathcal{T}$ and the corresponding runs $\widehat{r}$ and $\widehat{r}_{3}$ in $\widehat{\mathcal{T}}^{\text {td }}$ and $\widehat{\mathcal{T}}_{3 b l}^{\Omega^{\mathrm{td}}}$ respectively. The run $\widehat{r}$ belongs to the objective $\operatorname{TimeDivBl}_{1}(\operatorname{Parity}(\Omega))=(\square \diamond$ tick $\rightarrow \operatorname{Parity}(\Omega)) \wedge\left(\neg \square \diamond\right.$ tick $\left.\rightarrow \diamond \square \neg b l_{1}\right)$ iff the run $\widehat{r}_{3}$ belongs to the objective TimeDiv3B $I_{1}(\operatorname{Parity}(\Omega))=(\square \diamond$ tick $\rightarrow \operatorname{Parity}(\Omega)) \wedge(\neg \square \diamond$ tick $\rightarrow$ $\left.\diamond \square \neg 3 b l_{1}\right)$.

Proof. We have that for all $k \geq 0$, except for the blame component, the remaining four corresponding components of the 5 -tuples $\widehat{r}[k]$ and of $\widehat{r}_{3}[k]$ match. Hence the membership of the run $\widehat{r}$ in the objective $\operatorname{TimeDivBl}_{1}(\operatorname{Parity}(\Omega))$ differs from the membership of the run $\widehat{r}_{3}$ in the objective TimeDiv3BI ${ }_{1}(\operatorname{Parity}(\Omega))$ only when both of the following conditions hold: (a) time converges on both runs (i.e., $(\square \diamond t i c k)=$ FALSE), and (b) $b l_{1}$ is true infinitely often in $\widehat{r}$ and $3 b l_{1}$ is true only finitely often in $\widehat{r}_{3}$; or vice versa. Observe that as the first four components of $\widehat{r}[k]$ and in $\widehat{r}_{3}[k]$ match, and both runs correspond to a run $r$ in $\mathcal{T}$, we have $b l_{1}^{k}$ to be true whenever $3 b l_{1}^{k}$ is true. Thus, we cannot have $3 b l_{1}$ to be true infinitely often in $\widehat{r}_{3}$ and $b l_{1}$ to be true only finitely often in $\widehat{r}$. Thus, we can restrict our attention to the case where $b l_{1}$ is true infinitely often, but $3 b l_{1}$ is only true finitely often. The $b l_{1}^{k}$ component of $\widehat{r}[k]$ differs from the $3 b l_{1}^{k}$ component in $\widehat{r}_{3}[k]$ for $k \geq 1$ only when a move $\left\langle\Delta^{k-1}, a_{1}^{k-1}\right\rangle$ of player 1 is chosen from the state $r[k-1]$ (and correspondingly from $\widehat{r}[k]$ and $\widehat{r}_{3}[k]$ ),

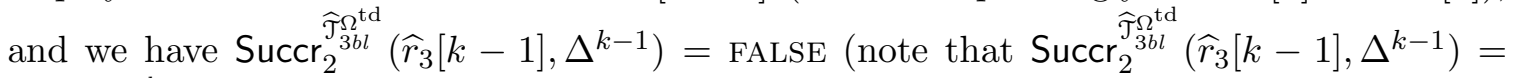
$\left.\operatorname{Succr}_{2}^{\widehat{\tau}^{\text {td }}}\left(\widehat{r}[k-1], \Delta^{k-1}\right)\right)$. Thus, we must have that player-1 moves $\left\langle\Delta^{k}, a_{1}^{k}\right\rangle$ were chosen from the state $\widehat{r}_{3}[k]$ such that $\operatorname{Succr}_{2}^{\widehat{\Im}^{\text {td }}}\left(\widehat{r}_{3}[k], \Delta^{k}\right)=$ FALSE for infinitely many $k$. Thus, from Lemma 3.2, we have the runs $\widehat{r}_{3}$ and $\widehat{r}$ to be time divergent. Hence, the membership of the run $\widehat{r}$ in the objective $\operatorname{TimeDivBl}_{1}(\operatorname{Parity}(\Omega))$ must be the same as the membership of

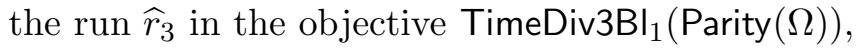

As in Lemma 2.5, the objective $\operatorname{TimeDiv} \mathrm{BI}_{1}(\operatorname{Parity}(\Omega))$ can be expressed as a parity objective Parity $\left(\Omega_{3 b l}^{\mathrm{td}}\right)$. Proposition 2.3 and Lemma 3.3 give us the following proposition which states that we can consider games in $\widehat{\mathcal{T}}_{3 b l}^{\Omega^{\text {td }}}$ to compute winning sets in $\mathcal{T}$.

Proposition 3.4. Consider a timed automaton game $\mathcal{T}$ and a location parity objective $\Omega$ on states of $\mathcal{T}$ with $\widehat{\mathcal{T}}_{3 b l}^{\Omega^{\mathrm{td}}}$ being the corresponding expanded game structure. Consider a run $r=$ $s^{0},\left\langle m_{1}^{0}, m_{2}^{0}\right\rangle, s^{1},\left\langle m_{1}^{1}, m_{2}^{1}\right\rangle, \ldots$ in $\mathcal{T}$. Let $\widehat{r}_{3}$ be the corresponding run in $\widehat{\mathcal{T}}_{3 b l}^{\Omega^{\text {td }}}$ such that $\widehat{r}_{3}=$ $\left\langle s^{0}, \mathfrak{z}^{0}, t i c k^{0}, b l_{1}^{0}, p^{0}\right\rangle,\left\langle m_{1}^{0}, m_{2}^{0}\right\rangle,\left\langle s^{1}, \mathfrak{z}^{1}, t i c k^{1}, b l_{1}^{1}, p^{1}\right\rangle,\left\langle m_{1}^{1}, m_{2}^{1}\right\rangle$ with $\mathfrak{z}^{0}=0$, tick $k^{0}=$ FALSE, $b l_{1}^{0}=$ FALSE, $p^{0}=0$. Then $r \in \operatorname{TimeDivBl}_{1}(\operatorname{Parity}(\Omega))$ iff $\widehat{r}_{3} \in \operatorname{TimeDiv}_{3} \mathrm{Bl}_{1}(\operatorname{Parity}(\Omega))$ (i.e., $\widehat{r}_{3} \in=\operatorname{Parity}\left(\Omega_{3 b l}^{\mathrm{td}}\right)$, or equivalently, $\widehat{r}_{3} \in(\square \diamond$ tick $\rightarrow$ Parity $(\Omega)) \wedge\left(\neg \square \diamond\right.$ tick $\left.\rightarrow \diamond \square \neg 3 b l_{1}\right)$. 
3.1.3. Sufficiency of 3-region strategies. We next show that we can restrict both players to use only 3-region strategies in the game structure $\widehat{\mathcal{T}}_{3 b l}^{\Omega^{\text {td }}}$ without changing the winning sets. We first consider 3-region strategies of player 1 . The following lemma is a key lemma of the paper and an example is presented after the proof to help illustrate its workings.

Lemma 3.5. Consider a timed automaton game $\mathcal{T}$ with $\Omega$ a location parity function on states of $\mathcal{T}$ and $\widehat{\mathcal{T}}_{3 b l}^{\Omega^{\mathrm{td}}}$ the corresponding expanded game structure. Then, 3-region memoryless region strategies of player 1 suffice for winning from $\operatorname{Win}_{1}^{\widehat{\mathcal{T}}_{3 b l}^{\Omega^{\mathrm{td}}}}\left(\operatorname{TimeDiv3BI_{1}}(\operatorname{Parity}(\Omega))\right)$.

Proof. As in Lemma 2.7, let $\pi_{1}$ be a player-1 winning memoryless region strategy in $\widehat{\mathcal{T}}_{3 b l}^{\Omega^{\mathrm{td}}}$ for $\left.\operatorname{TimeDiv3BI_{1}(\operatorname {Parity}(\Omega )}\right)$ (the results of Lemma 2.7, also hold for the structure $\widehat{\mathcal{T}}_{3 b l}^{\Omega^{\mathrm{td}}}$ ). We construct a 3-region memoryless region strategy $\pi_{1}^{*}$ of player 1 which wins against all strategies of player 2 . Intuitively, from a state $\widehat{s}$, the strategy $\pi_{1}^{*}$ prescribes (a) the same move as $\pi_{1}$ when $\pi_{1}$ prescribes a move to either the current region or to the two immediately succeeding regions (b) prescribes a relinquishing move $\perp_{*}$ when $\pi$ prescribes a relinquishing move, and (c) prescribes a time blocking move to the second region following $\operatorname{Reg}(\widehat{s})$ whenever $\pi_{1}$ proposes a move to outside the second succeeding region. Formally, let $\widehat{r}_{3}$ be any run in $\widehat{\mathcal{T}}_{3 b l}^{\Omega \text { td }}$. The strategy $\pi_{1}^{*}$ is specified by:

$$
\pi_{1}^{*}\left(\widehat{r}_{3}[0 . . k]\right)= \begin{cases}\left\langle\Delta, a_{1}\right\rangle & \text { if } \pi_{1}\left(\widehat{r}_{3}[0 . . k]\right)=\left\langle\Delta, a_{1}\right\rangle \text { and } \operatorname{Succr}_{3} \widehat{\widetilde{T}}_{3 b l}^{\Omega^{\mathrm{td}}}\left(\widehat{r}_{3}[k], \Delta\right)=\mathrm{TRUE}, \\ & \text { and } a_{1} \neq \perp_{*} \\ \left\langle 0, \perp_{*}\right\rangle & \text { if } \pi_{1}\left(\widehat{r}_{3}[0 . . k]\right)=\left\langle\Delta, \perp_{*}\right\rangle \\ \left\langle\Delta, \perp_{1}\right\rangle & \text { if } \pi_{1}\left(\widehat{r}_{3}[0 . . k]\right)=\left\langle\Delta_{1}, a_{1}\right\rangle, a_{1} \neq \perp_{*}, \text { and } \\ & \widehat{\widehat{T}}_{3 b l}^{\Omega^{\mathrm{td}}}\left(\widehat{r}_{3}[k], \Delta_{1}\right)=\text { FALSE; where } \Delta \text { is any real number } \\ & \text { Succr } \\ & \text { such that }\left|\left\{\operatorname{Reg}\left(\widehat{r}_{3}[k]+\Delta^{\prime}\right) \mid 0 \leq \Delta^{\prime} \leq \Delta\right\}\right|=3 .\end{cases}
$$

Note that $\pi_{1}^{*}$ is a memoryless region strategy as $\pi_{1}$ is a memoryless region strategy.

We claim $\pi_{1}^{*}$ wins against all strategies of player 2 for every state $\widehat{s} \in \operatorname{Win}_{1}^{\widehat{\tau}_{3 b l}^{\Omega^{t d}}}$. Suppose this is not true. Let $\pi_{2}^{*}$ be a spoiling move-independent region strategy of player 2 against $\pi_{1}^{*}$ from $\widehat{s} \in \mathrm{Win}_{1}^{\widehat{\mathfrak{T}}^{\Omega \text { td }}}$ (move-independent region strategies suffice as spoiling strategies by a lemma corresponding to Lemma 2.9 for the structure $\widehat{\mathcal{T}}_{3 b l}^{\Omega^{\mathrm{td}}}$ ). Suppose $\widehat{r}_{3}^{*} \in$ Outcomes $\left(\widehat{s}, \pi_{1}^{*}, \pi_{2}^{*}\right)$ and $\widehat{r}_{3}^{*} \notin \operatorname{TimeDiv} 3 \mathrm{Bl}_{1}(\operatorname{Parity}(\Omega))$. We show that in that case we can construct a player-2 spoiling strategy for $\pi_{1}$, contrary to the assumption that $\pi_{1}$ was a winning strategy. Intuitively, given a finite run $\widehat{r}_{3}[0 . . k]$ the strategy $\pi_{2}$ :

(1) Acts like $\pi_{2}^{*}$ when $\pi_{1}$ proposes a relinquishing move.

(2) Acts like $\pi_{2}^{*}$ when $\pi_{1}$ proposes moves within three regions.

(3) Acts like $\pi_{2}^{*}$ when $\pi_{1}$ proposes moves outside three regions and $\pi_{2}^{*}$ proposes moves of shorter duration than $\pi_{1}^{*}$ (observe that $\pi_{1}^{*}$ moves are pure time moves in case $\pi_{1}$ proposes moves outside three regions).

(4) Proposes the same time delay moves as $\pi_{1}^{*}$ when $\pi_{1}$ proposes moves outside three regions, and $\pi_{2}^{*}$ proposes moves longer than $\pi_{1}^{*}$;

(5) Proposes the same moves as $\pi_{2}^{*}$ when $\pi_{1}$ proposes moves outside three regions, $\pi_{2}^{*}$ proposes moves of exactly the same duration as $\pi_{1}^{*}$, and the move of $\pi_{2}^{*}$ is chosen at $\widehat{r}_{3}^{*}[k+1]$. 
(6) Proposes the same time delay moves as $\pi_{1}^{*}$ when $\pi_{1}$ proposes moves outside three regions and when $\pi_{2}^{*}$ proposes moves of exactly the same duration as $\pi_{1}^{*}$ if the move of $\pi_{1}^{*}$ is chosen at $\widehat{r}_{3}^{*}[k+1]$.

Formally, the player-2 strategy $\pi_{2}$ (dependent on the strategies $\pi_{1}, \pi_{1}^{*}$ and $\pi_{2}^{*}$ ) is defined to be:

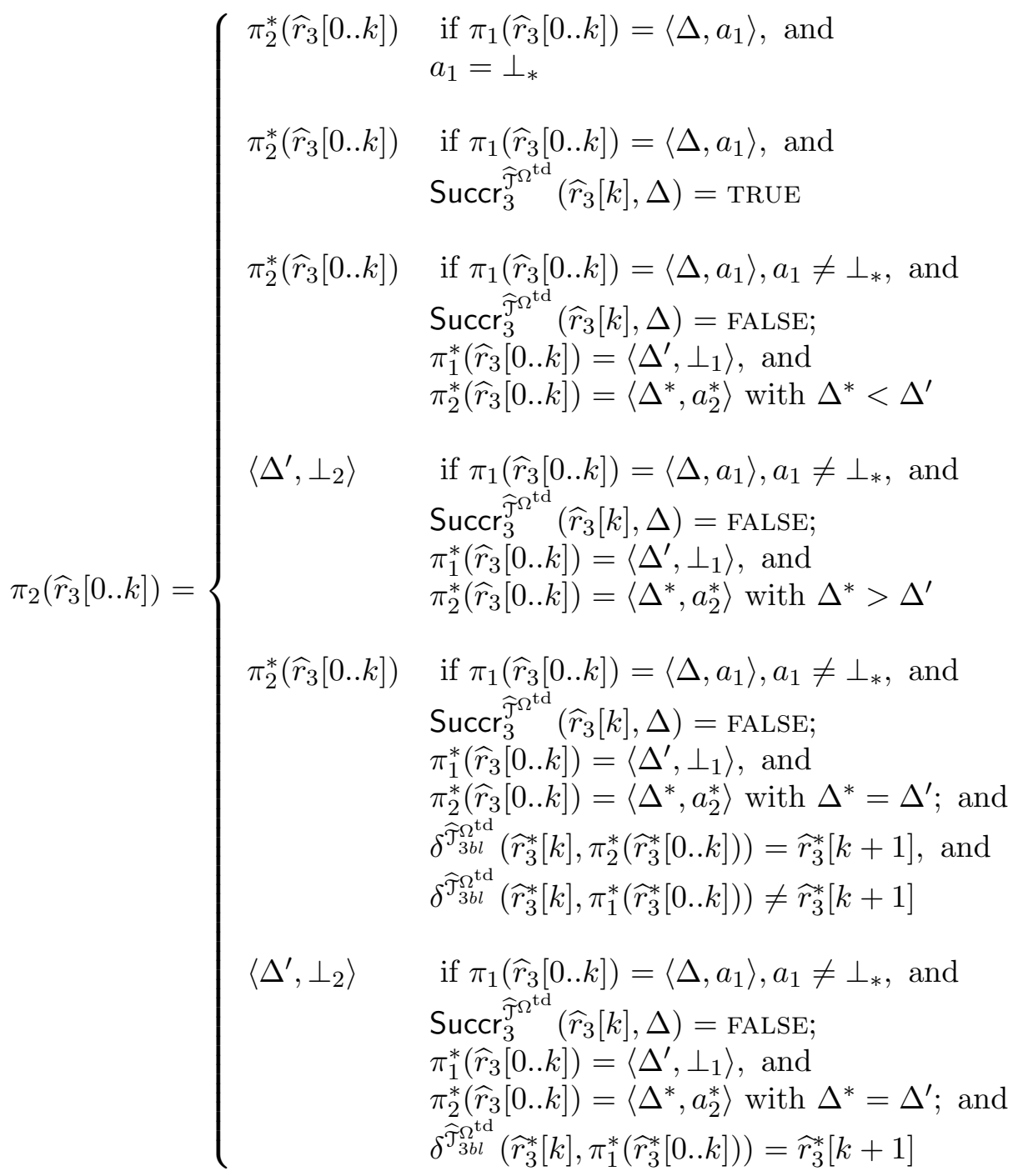

Note that $\pi_{2}$ is a memoryless strategy (as $\pi_{1}, \pi_{1}^{*}, \pi_{2}^{*}$ are all memoryless). We show that

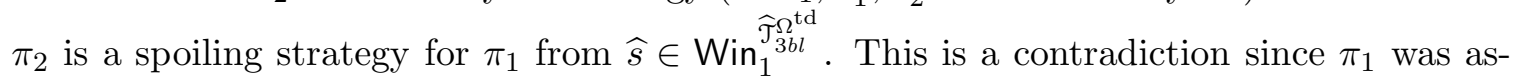
sumed to be a player-1 winning strategy. We show there exists a run $\widehat{r}_{3} \in \operatorname{Outcomes}\left(\widehat{s}, \pi_{1}, \pi_{2}\right)$ such that $\widehat{r}_{3}[k]=\widehat{r}_{3}^{*}[k]$ for all $k \geq 0$ (recall that $\widehat{r}_{3}^{*}$ is the run used in defining $\pi_{2}$, and is

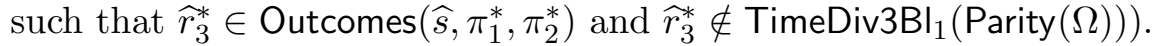

We proceed by induction on $k$. For $k=0$ the claim is trivially true. Suppose the claim is true for all $j \leq k$. Thus, we have a run $\widehat{r}_{3}$ such that $\widehat{r}_{3}[j]=\widehat{r}_{3}^{*}[j]$ for all $j \leq k$. We show that the run $\widehat{r}_{3}[0 . . k]$ can be extended to $\widehat{r}_{3}[0 . . k+1]$ according to $\pi_{1}, \pi_{2}$ such that $\widehat{r}_{3}[k+1]=\widehat{r}_{3}^{*}[k+1]$. Informally, we have the following cases (we can ignore the moves taken 
and only focus on the states in the runs $\widehat{r}_{3}[0 . . k]$, which is the same as the states in the run $\widehat{r}_{3}^{*}[0 . . k]$ since $\pi_{1}, \pi_{2}, \pi_{1}^{*}, \pi_{2}^{*}$ are all memoryless and hence move independent):

(1) The strategies $\pi_{1}$ and $\pi_{1}^{*}$ propose a relinquishing move for the state sequence of $\widehat{r}_{3}[0 . . k]$ (which is the same as the state sequence $\widehat{r}_{3}^{*}[0 . . k]$ by the inductive hypothesis). In this case the move proposed by player 2 is the same for $\pi_{2}$ and for $\pi_{2}^{*}$, so the next state $\widehat{r}_{3}[k+1]$ is the same as $\widehat{r}_{3}^{*}[k+1]$.

(2) For the state sequence $\widehat{r}_{3}[0 . . k]$, the strategies $\pi_{1}$ and $\pi_{1}^{*}$ propose the same non-relinquishing move to either the current region, or the next two immediately succeeding regions. In this case the move proposed by player 2 is the same for $\pi_{2}$ and for $\pi_{2}^{*}$, so the next state $\widehat{r}_{3}[k+1]$ is the same as $\widehat{r}_{3}^{*}[k+1]$.

(3) The strategy $\pi_{1}$ proposes a move outside the second succeeding region, $\pi_{1}^{*}$ proposes a delay move to the second succeeding region, and $\pi_{2}^{*}$ proposes a move shorter than $\pi_{1}^{*}$. In this case, the strategy $\pi_{2}$ proposes the same move as $\pi_{2}^{*}$, and hence the same move of player 2 determines both $\widehat{r}_{3}[k+1]$ and $\widehat{r}_{3}^{*}[k+1]$. Thus, the two states are equal.

(4) The strategy $\pi_{1}$ proposes a move outside the second succeeding region, $\pi_{1}^{*}$ proposes a delay move to the second succeeding region, and $\pi_{2}^{*}$ proposes a move longer than $\pi_{1}^{*}$. In this case, the strategy $\pi_{2}$ proposes the same delay move as $\pi_{1}^{*}$, and hence the state $\widehat{r}_{3}[k+1]$ is the same as $\widehat{r}_{3}^{*}[k+1]$ as both states are determined by delay moves of equal duration. The $3 b l_{1}$ component remains FALSE in both $\widehat{r}_{3}[k+1]$ and $\widehat{r}_{3}^{*}[k+1]$ as the transition is outside the immediately succeeding region.

(5) The strategy $\pi_{1}$ proposes a move outside the second succeeding region, $\pi_{1}^{*}$ proposes a delay move to the second succeeding region, $\pi_{2}^{*}$ proposes a move of exactly the same duration as $\pi_{1}^{*}$, and the move of player 2 according to the strategy $\pi_{2}^{*}$ determines $\widehat{r}_{3}^{*}[k+1]$. In this case $\pi_{2}$ behaves like $\pi_{2}^{*}$, and hence the state $\widehat{r}_{3}[k+1]$ is the same as $\widehat{r}_{3}^{*}[k+1]$ as both are determined by the same move of player 2 .

(6) The strategy $\pi_{1}$ proposes a move outside the second succeeding region, $\pi_{1}^{*}$ proposes a delay move to the second succeeding region, $\pi_{2}^{*}$ proposes a move of exactly the same duration as $\pi_{1}^{*}$, and the delay move of player 1 according to $\pi_{1}^{*}$ determines the next state $\widehat{r}_{3}^{*}[k+1]$. In this case $\pi_{2}$ proposes a delay move of the same duration as $\pi_{1}^{*}$ and hence the state $\widehat{r}_{3}[k+1]$ is the same as $\widehat{r}_{3}^{*}[k+1]$ as both are determined by delay moves of equal duration. The $3 b l_{1}$ component remains FALSE in both $\widehat{r}_{3}[k+1]$ and $\widehat{r}_{3}^{*}[k+1]$ as the transition is outside the immediately succeeding region.

The full details can be found in the appendix.

Thus, in all cases, we have that $\widehat{r}_{3}[0 . . k]$ can be extended to $\widehat{r}_{3}[0 . . k+1]$ according to $\pi_{1}, \pi_{2}$ such that $\widehat{r}_{3}[0 . . k+1]=\widehat{r}_{3}^{*}[0 . . k+1]$. Hence, we have $\widehat{r}_{3} \in \operatorname{Outcomes}\left(\widehat{s}, \pi_{1}, \pi_{2}\right)$ and

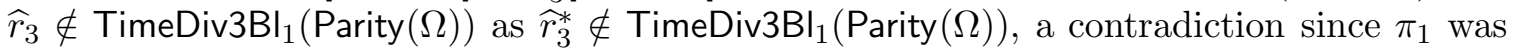
assumed to be a winning strategy. Hence, we cannot have the existence of the strategy $\pi_{2}^{*}$ from which $\widehat{r}_{3}^{*}$ and $\pi_{2}$ were derived, i.e., $\pi_{1}^{*}$ is a winning strategy for player 1 from $\widehat{s}$.

The next example illustrates the above lemma and the usefulness of the structure $\widehat{\mathcal{T}}_{3 b l}^{\Omega \text { td }}$.

Example 3.6. Consider the timed automaton $\mathcal{T}$ in Figure 4. Suppose the objective of player 1 is to reach the location $l^{1}$ starting from $l^{0}$. Player 1 controls only the edge $a_{1}$. Player 2 controls the other edges $a_{2}^{1}$ and $a_{2}^{2}$. Player 1 wins from $l^{0}$ so long as $x<4$. Let $r[0 . . k]$ be a run such that $\alpha_{x}<4$ where $\alpha_{x}$ is the value of clock $x$ in state $r[k]$. A winning 


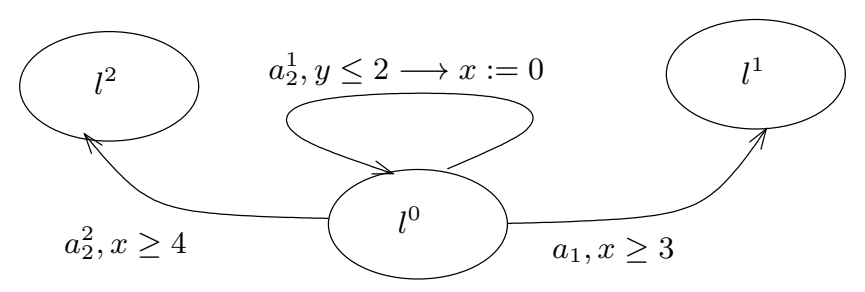

Figure 4: A timed automaton game $\mathcal{T}$.

strategy for player 1 is given by

$$
\pi_{1}(r[0 . . k])=\begin{array}{ll}
\left\langle 3-\alpha_{x}, a_{1}\right\rangle & \text { if } \alpha_{x} \leq 3 \\
\left\langle 0, a_{1}\right\rangle & \text { otherwise }
\end{array}
$$

Based on $\pi_{1}$, a 3-region winning strategy $\pi_{1}^{*}$ can be obtained as in Lemma 3.5. Intuitively, from a state $\widehat{s}$, the strategy $\pi_{1}^{*}$ prescribes (a) the same move as $\pi_{1}$ when $\pi_{1}$ prescribes a move to either the current region or to the two immediately succeeding regions, and (b) prescribes a time elapsing move to the second region following $\operatorname{Reg}(\widehat{s})$ whenever $\pi_{1}$ proposes a move to outside the second succeeding region.

Let the initial state be $\widehat{s}_{0}=\left\langle l^{0}, z=x=y=0\right.$, tick = FALSE, $3 b l_{1}=$ FALSE $\rangle$ (we skip the parity component for simplicity). We consider a sample play from this state. Initially, $\pi_{1}^{*}$ proposes the move $\left\langle 1, \perp_{1}\right\rangle$ from $\widehat{s}_{0}$. Suppose it is allowed by player 2 . The resulting state is then $\widehat{s}_{1}=\left\langle l^{0}, z=0, x=y=1\right.$, tick $=$ TRUE, $3 b l_{1}=$ FALSE $\rangle$. In the second step, $\pi_{1}^{*}$ again proposes the move $\left\langle 1, \perp_{1}\right\rangle$ from $\widehat{s}_{1}$. the resulting state is $\widehat{s}_{2}=\left\langle l^{0}, z=0, x=y=2\right.$, tick $=$ TRUE, $3 b l_{1}=$ FALSE $\rangle$. In the third step, $\pi_{1}^{*}$ proposes the move $\left\langle 1, a_{1}\right\rangle$ which is blocked by the player-2 move $\left\langle 0, a_{2}^{1}\right\rangle$, leading to the state $\widehat{s}_{3}=\left\langle l^{0}, z=0, x=0, y=2\right.$, tick $=$ FALSE, $3 b l_{1}=$ FALSE $\rangle$. Player 2 can then take the action $a_{2}^{1}$ a finite number of times, but eventually it must let time elapse. When it does, player 1 is able to take the action $a_{1}$ in three steps.

Now we show that there exists a player-2 strategy $\pi_{2}$ such that $\pi_{1}$ and $\pi_{2}$ result in the same sequence of states $\widehat{s}_{0}, \widehat{s}_{1}, \widehat{s}_{2}, \ldots$ The strategy $\pi_{2}$ proposes a time elapsing move $\left\langle 1, \perp_{2}\right\rangle$ from $\widehat{s}_{0}$ and then again from $\widehat{s}_{1}$. It then proposes $\left\langle 0, a_{2}^{1}\right\rangle$ from $\widehat{s}_{2}$ as against $\pi_{1}^{*}$, and then again $\left\langle 1, \perp_{2}\right\rangle$ for one more step. It can be verified that this leads to the same sequence of states as with $\pi_{1}^{*}$.

Suppose we had been working in the structure $\widehat{\mathcal{T}}^{\Omega^{\text {td }}}$. Then the states $\widehat{s}_{1}$ and $\widehat{s}_{2}$ would have had $b l_{1}=$ TRUE. Thus, in reaching $l^{1}$, the strategy $\pi_{1}^{*}$ would have resulted in some states having $b l_{1}=$ TRUE, in contrast to the strategy $\pi_{1}$ which manages to have $b l_{1}=$ FALSE until $l^{1}$ is reached. Thus $\pi_{1}$ and $\pi_{1}^{*}$ would on the surface appear to be inherently incompatible with respect to objectives. In this example however, it can be seen that we may as well work with $3 b l_{1}$ as player- 1 time-elapsing moves can be chosen only finitely often, thus $3 b l_{1}$ and $b l_{1}$ differ only finitely often.

Next, we show that 3-region memoryless strategies of player 2 suffice as spoiling strategies. An example will be presented after the lemma to illustrate the sufficiency of 3-region strategies.

Lemma 3.7. Consider a timed automaton game $\mathcal{T}$ and a location parity objective $\Omega$ on states of $\mathcal{T}$ with $\widehat{\mathcal{T}}_{3 b l}^{\Omega^{\mathrm{td}}}$ being the corresponding expanded game structure. Suppose $\widehat{s} \notin$ $\mathrm{Win}_{1}^{\widehat{\mathcal{T}}_{3 b l}^{\text {td }}}\left(\operatorname{TimeDiv}_{\mathrm{B}} \mathrm{Bl}_{1}(\operatorname{Parity}(\Omega))\right)$. Then, 3-region memoryless region strategies of player 2 suffice for preventing player 1 from satisfying the objective TimeDiv3BI ${ }_{1}(\operatorname{Parity}(\Omega))$ from $\widehat{s}$. 
Proof. The objective TimeDiv3BI 1 (Parity $(\Omega)$ ) corresponds to the parity objective $\operatorname{Parity}\left(\Omega^{\text {td }}\right)$ by Lemma 2.5 (replacing $b l_{1}$ by $3 b l_{1}$ ). As mentioned in Section 2 , there exists a game $\mu$ calculus formula which precisely characterizes the winning set $\operatorname{Win}_{1}^{\widehat{\mathcal{T}}^{3 b l}}\left(\operatorname{Parity}\left(\Omega^{\mathrm{td}}\right)\right)$. The winning sets can be obtained by a $\mu$-calculus iteration. The iteration uses the controllable predecessor operator for player 1, CPre $: 2^{\widehat{S}} \mapsto 2^{\widehat{S}}$ (where $\widehat{S}$ is the state space of $\widehat{\mathcal{T}}_{3 b l}^{\Omega^{\text {td }}}$ ), defined formally by $\widehat{s} \in \mathrm{CPre}_{1}(Z)$ iff $\exists m_{1} \in \Gamma_{1}^{\widehat{\mathcal{T}}_{3 b l}^{\Omega \mathrm{td}}}(\widehat{s}) \forall m_{2} \in \Gamma_{2}^{\widehat{\tau}_{3 b l}^{\Omega^{\mathrm{td}}}}(\widehat{s}) \cdot \delta_{\mathrm{jd}}^{\widehat{\mathcal{T}}_{3 b l}^{\Omega^{\mathrm{td}}}}\left(\widehat{s}, m_{1}, m_{2}\right) \subseteq Z$. Informally, $\mathrm{CPre}_{1}(Z)$ consists of the set of states from which player 1 can ensure that the next state will be in $Z$, no matter what player 2 does. It can be shown that $\mathrm{CPre}_{1}$ preserves regions of $\widehat{\mathcal{T}}_{3 b l}^{\Omega^{\mathrm{td}}}$ using Lemma 3.1. The iteration also suggests winning strategies for player 1 based on the sets that arise in the iteration. The sets that arise depend on the parity labeling, the $\mathrm{CPre}_{1}$ and the fixpoint operators. If we have a location parity objective, it can be shown that only unions of regions arise as sets in the fixpoint iteration. To prove that 3-region memoryless region strategies of player 2 suffice as spoiling strategies, it hence suffices to show that the $\mathrm{CPre}_{1}$ sets of unions of regions remains unchanged if we restrict player-2 strategies to be 3-region memoryless region strategies.

Let $\mathrm{CPre}_{1,3}(Z)$ denote the set of states from which player 1 can ensure that the next state will be in $Z$, no matter what move player 2 takes within 3 regions. Also, clearly $\operatorname{CPre}_{1,3}(Z)$ depends only on $Z$, and not on the history of the game (hence we shall have memoryless 3-region spoiling strategies). We show $\mathrm{CPre}_{1,3}(Z)=\mathrm{CPre}_{1}(Z)$ for $Z$ a union of regions. Clearly $\mathrm{CPre}_{1}(Z) \subseteq \mathrm{CPre}_{1,3}(Z)$ as player 2 has fewer moves to counter with in $\mathrm{CPre}_{1,3}(Z)$. To prove the other direction, we show if $\widehat{s} \in \mathrm{CPre}_{1,3}(Z)$, then $\widehat{s} \in \mathrm{CPre}_{1}(Z)$. We first characterize the $\mathrm{CPre}_{1}$ sets. A state $\widehat{s} \in \mathrm{CPre}_{1}(Z)$ iff either one of the following conditions is met:

(1) $\left\{\delta\left(\widehat{s},\left\langle\Delta, a_{2}\right\rangle\right) \mid\left\langle\Delta, a_{2}\right\rangle \in \Gamma_{2}(\widehat{s})\right\} \subseteq Z$.

(2) There exists $\left\langle\Delta, a_{1}\right\rangle \in \Gamma_{1}(\widehat{s})$ with $a_{1} \neq \perp_{*}$ such that

(a) $\delta\left(\widehat{s},\left\langle\Delta, a_{1}\right\rangle\right) \in Z$, and

(b) $\left\{\delta\left(\widehat{s},\left\langle\Delta^{\prime}, a_{2}\right\rangle\right) \mid \Delta^{\prime} \leq \Delta\right.$, and $\left.\left\langle\Delta^{\prime}, a_{2}\right\rangle \in \Gamma_{2}(\widehat{s})\right\} \subseteq Z$.

The first condition corresponds to the case when player 1 proposes a move $\left\langle\Delta, \perp_{*}\right\rangle$ from $\widehat{s}$. In this case, the move of player 2 will be chosen, no matter the move. Thus, we must have that no matter the move of player 2 , the resultant state must be in $Z$. The second condition corresponds to the case when player 1 proposes a move $\left\langle\Delta, a_{1}\right\rangle$ from $\widehat{s}$ with $a_{1} \neq \perp_{*}$. In this case, every move $\left\langle\Delta^{\prime}, a_{1}\right\rangle$ of player 2 with $\Delta^{\prime} \leq \Delta$ must lead to $Z$. A similar characterization exists for $\mathrm{CPre}_{1,3}(Z)$, the only difference being that player-2 moves are restricted to be within 3 regions: a state $\widehat{s} \in \mathrm{CPre}_{1,3}(Z)$ iff either one of the following conditions is met:

(1) $\left\{\delta\left(\widehat{s},\left\langle\Delta, a_{2}\right\rangle\right) \mid\left\langle\Delta, a_{2}\right\rangle \in \Gamma_{2}(\widehat{s})\right.$ and $\operatorname{Succr}_{3}(\widehat{s}, \Delta)=$ TRUE $\} \subseteq Z$.

(2) There exists $\left\langle\Delta, a_{1}\right\rangle \in \Gamma_{1}(\widehat{s})$ with $a_{1} \neq \perp_{*}$ such that

(a) $\delta\left(\widehat{s},\left\langle\Delta, a_{1}\right\rangle\right) \in Z$, and

(b) $\left\{\delta\left(\widehat{s},\left\langle\Delta^{\prime}, a_{2}\right\rangle\right) \mid \Delta^{\prime} \leq \Delta\right.$, $\operatorname{Succr}_{3}\left(\widehat{s}^{*}, \Delta^{\prime}\right)=$ TRUE, and $\left.\left\langle\Delta^{\prime}, a_{2}\right\rangle \in \Gamma_{2}(\widehat{s})\right\} \subseteq Z$.

Now we show that if $\widehat{s} \in \mathrm{CPre}_{1,3}(Z)$, then $\widehat{s} \in \mathrm{CPre}_{1}(Z)$. Suppose $\widehat{s} \in \mathrm{CPre}_{1,3}(Z)$. Informally, we can have the following cases (the formal details are in the appendix):

- The available moves of player 2 are only until the second immediately succeeding region. In this case, the restriction of player-2 strategies to be 3-region strategies has no effect, hence $\widehat{s} \in \mathrm{CPre}_{1}(Z)$. 


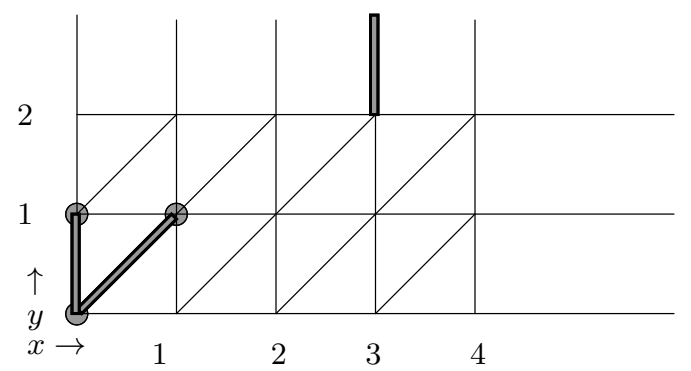

Figure 5: Regions of the timed automaton game $\mathcal{T}$ of Figure 4 .

- Player 2 has a move from $\widehat{s}$ to a state that is farther than the second succeeding region. This means that it has a delay move to the second succeeding region. In this case, player 1 can play the same delay move to the second succeeding region. The resulting state will be the same as due to the delay move of player 2 to the second succeeding region, because $3 b l_{1}$ will be false in both cases. Moreover, all moves of player 2 which lie in the current or the next two regions result in a state in $Z$ by assumption. In particular, the delay move of player 1 will also result in a state in $Z$. Thus, $\widehat{s} \in \mathrm{CPre}_{1}(Z)$.

Thus, in all cases $\widehat{s} \in \mathrm{CPre}_{1}(Z)$ whenever $\widehat{s} \in \mathrm{CPre}_{1,3}(Z)$.

Example 3.8. Consider the timed automaton game in Figure 4 and the following regions.

$$
\begin{aligned}
& \widehat{R}_{5}=\left\{\left\langle l^{1}, z=0, x=3, y, \text { tick }=\mathrm{TRUE}, 3 b l_{1}=\mathrm{FALSE}\right\rangle \mid y>2\right\} \\
& \widehat{R}_{4}=\left\{\left\langle l^{0}, z=0, x=y=1, \text { tick }=\mathrm{TRUE}, 3 b l_{1}=\mathrm{FALSE}\right\rangle\right\} \\
& \widehat{R}_{3}=\left\{\left\langle l^{0}, z=x=y, \text { tick }=\mathrm{FALSE}, 3 b l_{1}=\mathrm{FALSE}\right\rangle \mid 0<x<1\right\} \\
& \widehat{R}_{2}=\left\{\left\langle l^{0}, z=0, x=0, y=1, \text { tick }^{0}=\mathrm{TRUE}, 3 b l_{1}=\mathrm{FALSE}\right\rangle\right\} \\
& \widehat{R}_{1}=\left\{\left\langle l^{0}, z=y, x=0, \text { tick }=\mathrm{FALSE}, 3 b l_{1}=\mathrm{FALSE}\right\rangle \mid 0<y<1\right\} \\
& \widehat{R}_{0}=\left\{\left\langle l^{0}, z=0, x=y=0, \text { tick }=\mathrm{FALSE}, 3 b l_{1}=\mathrm{FALSE}\right\rangle\right\}
\end{aligned}
$$

The regions can be seen as the thick lines and dots in Figure 5 (the clock $z$ is not shown for simplicity). Let $Z=\bigcup_{j=0}^{5} \widehat{R}_{j}$.

The state $\widehat{s}_{0}=\left\langle l^{0}, z=0, x=y=0\right.$, tick $=$ FALSE, $3 b l_{1}=$ FALSE $\rangle$ belongs to $\operatorname{CPre}_{1,3}(Z)$. This can be seen as follows. From state $\widehat{s}_{0}$, player 1 proposes the move $\left\langle 3, a_{1}\right\rangle$. Player 2 can only propose a counter move $\left\langle\Delta, a^{*}\right\rangle$ such that $0 \leq \Delta \leq 1$ and $a^{*} \in\left\{\perp_{2}, a_{2}^{1}\right\}$. Now observe that $\widehat{s}_{0}$ also belongs to $\mathrm{CPre}_{1}(Z)$. To see this, we have to consider a different player-1 move, namely $\left\langle 1, \perp_{1}\right\rangle$. If player 2 allows this move of player 1 , the next state will be in $\widehat{R}_{4}$ (the variable $3 b l_{1}$ remains false as the allowed move of player 1 is not in the immediate successor region of $\widehat{s}_{0}$ ). If player 2 instead proposes a shorter (or equal) duration move, the next state will be in $\bigcup_{j=0}^{4} \widehat{R}_{j}$.

Let $Z^{*}$ be any set of states which includes $\widehat{R}_{5}$. Suppose $\widehat{s}_{0} \in \mathrm{CPre}_{1,3}\left(Z^{*}\right)$ or $\widehat{s}_{0} \in$ $\mathrm{CPre}_{1}\left(Z^{*}\right)$. Then it can be seen that $Z^{*}$ must contain the states from $Z$. Hence, we have $\widehat{s}_{0} \in \mathrm{CPre}_{1,3}\left(Z^{*}\right)$ iff $\widehat{s}_{0} \in \mathrm{CPre}_{1}\left(Z^{*}\right)$. In general, 3-region strategies of player 2 suffice as if there exists a move of player 2 beyond the two immediately succeeding regions, then both player 1 and player 2 have simple time passage moves to the second succeeding region, with 
the variable $3 b l_{1}$ remaining false if any of these two moves is chosen. To mimic a general player-2 counter move, a 3-region strategy of player 2 can play simple time passage moves to the second successor regions till it gets to a state from which a desired player-2 edge lies within two successor regions.

Given an objective $\operatorname{Parity}(\Omega)$, let $\operatorname{Win}_{1}^{3, \widehat{\mathcal{T}}_{3}}(\operatorname{Parity}(\Omega))$ denote the states in the expanded game structure $\widehat{\mathcal{T}}_{3}$ such that for all states $\widehat{s} \in \mathrm{Win}_{1}^{3, \widehat{\mathcal{T}}_{3}}(\operatorname{Parity}(\Omega))$, player 1 has a 3 -region strategy $\pi_{1}$ such that for all 3-region strategies $\pi_{2}$ of player 2 , we have Outcomes $\left(\widehat{s}, \pi_{1}, \pi_{2}\right) \subseteq$ Parity $(\Omega)$. The following theorem, which follows from Lemmas 3.5, 3.7, and 3.3 states that to solve for $\operatorname{TimeDiv3BI_{1}}(\operatorname{Parity}(\Omega))$ in $\widehat{\mathcal{T}}_{3 b l}^{\Omega^{\mathrm{td}}}$, we can restrict both players to use only 3-region strategies. We call such games 3-region parity games.

Theorem 3.9. Consider a timed automaton game $\mathcal{T}$ and a location parity objective $\Omega$ on states of $\mathcal{T}$ with $\widehat{\mathcal{T}}_{3 b l}^{\Omega^{\mathrm{td}}}$ being the corresponding expanded game structure. We have

$$
\begin{aligned}
& \left.\operatorname{Win}_{1}^{3, \widehat{\mathcal{T}}_{3 b l}^{\Omega^{\text {td }}}(\operatorname{TimeDiv3BI}}{ }_{1}(\operatorname{Parity}(\Omega))\right)=\operatorname{Win}_{1}^{\widehat{\mathcal{T}}_{3 b l}^{\text {td }}}\left(\operatorname{TimeDiv3BI}_{1}(\operatorname{Parity}(\Omega))\right) \\
& =\operatorname{Win}_{1}^{\widehat{\mathcal{T}}_{3 b l}^{\Omega^{\mathrm{td}}}}\left(\operatorname{TimeDivBl}_{1}(\operatorname{Parity}(\Omega))\right) \text {. }
\end{aligned}
$$

\subsection{Reduction from 3-Region Parity Games to Finite-State Turn-Based Parity} Games. We gave a rough construction of a bipartite turn-based game to capture the winning sets of timed parity games at the beginning of this section. The idea of the construction being that first player 1 proposes a move representing its intention to let time pass to get to an intermediate region of $\widehat{\mathcal{T}}_{3 b l}^{\Omega^{\text {td }}}$ and then take a discrete action from that region. This intermediate region together with the action and the originating region can be encoded as a player-2 state in the finite game as $\left\langle\widehat{R}, \widehat{R}^{\prime}, a_{1}\right\rangle$ with $\widehat{R}$ denoting the originating region, $\widehat{R}^{\prime}$ representing the intermediate region to let time pass to, and $a_{1}$ the player- 1 action to take from $\widehat{R}^{\prime}$. Note that this player-2 state $\left\langle\widehat{R}, \widehat{R}^{\prime}, a_{1}\right\rangle$ corresponds to an intermediate step in the game $\widehat{\mathcal{T}}_{3 b l}^{\Omega^{\mathrm{td}}}$, where from a state $\widehat{s} \in \widehat{R}$ player 1 has just proposed its move (say $\left\langle\Delta_{1}, a_{1}\right\rangle$ with the time delay $\Delta$ leading to the region $\widehat{R}^{\prime}$ from which the discrete action $a_{1}$ will be taken. The game of $\widehat{\mathcal{T}}_{3 b l}^{\Omega^{\text {td }}}$ is still in the state $\widehat{s}$, waiting for the move from player 2 . In the finitestate game, from the player-2 state $\left\langle\widehat{R}, \widehat{R}^{\prime}, a_{1}\right\rangle$, player 2 then takes an action depending on whether it would allow the previously proposed player- 1 move from the originating region $\widehat{R}$ in $\widehat{\mathcal{T}}_{3 b l}^{\Omega^{\text {td }}}$ or not. This corresponds to the move of player 2 in $\widehat{\mathcal{T}}_{3 b l}^{\Omega^{\text {td }}}$ where it allows or disallows the player- 1 move $\left\langle\Delta_{1}, a_{1}\right\rangle$ from $\widehat{s}$ (hence the element of "surprise" from $\mathrm{dAFH}^{+} 03$ remains in this finite-state game). From Theorem 3.9, we can consider parity games where each player is restricted to use only 3-region strategies. This allows us to restrict the pairs of regions that can occur as player-2 states in the finite game.

Outline of subsection $\mathbf{3 . 2}$. We first present a finite-state turn-based game $\mathcal{T}_{\Omega}^{f}$ which can be used to compute the winning sets of $\mathcal{T}$. The size of the state space will be $O\left(\left|S_{\operatorname{Reg}}^{\widehat{\tau}_{3 b l}^{\text {td }}}\right| \cdot\left|A_{1}\right|\right)$ where $S_{\text {Reg }}^{\widehat{\mathcal{T}}_{3 b l}^{\mathrm{td}}}$ is the set of regions in $\widehat{\mathcal{T}}_{3 b l}^{\Omega^{\mathrm{td}}}$ and $A_{1}$ is the set of actions of player 1 in $\mathcal{T}$. We then 
present a finite-state turn-based game $\mathcal{T}_{\Omega}^{f *}$ such that (1) the winning set of $\mathcal{T}_{\Omega}^{f *}$ corresponds to that for $\mathcal{T}_{\Omega}^{f}$, and (2) the size of the state space of $\mathcal{T}_{\Omega}^{f *}$ is $O\left(\left|S_{\operatorname{Reg}}^{\widehat{\mathcal{T}}_{3 b l}^{\mathrm{td}}}\right|\right)$.

3.2.1. Construction of the finite turn-based bipartite game $\mathcal{T}_{\Omega}^{f}$. Given a timed automaton $\mathcal{T}$, and a location parity index function $\Omega$ on states of $\mathcal{T}$, the bipartite finite turn-based game $\mathcal{T}_{\Omega}^{f}$ consists of a tuple $\left\langle S^{f}, E^{f}, S_{1}^{f}, S_{2}^{f}\right\rangle$ where,

- $S^{f}=S_{1}^{f} \cup S_{2}^{f}$ is the state space. The states in $S_{i}^{f}$ are controlled by player- $i$ for $i \in\{1,2\}$.

- $S_{1}^{f}=\widehat{S}_{\text {Reg }} \times\{1\}$, where $\widehat{S}_{\text {Reg }}$ is the set of regions in $\widehat{\mathcal{T}}_{3 b l}^{\Omega^{\mathrm{td}}}$.

- $S_{2}^{f}=\widehat{S}_{\text {Tup }} \times\{2\}$, where $\widehat{S}_{\text {Tup }}=\widehat{S}_{\text {Reg }} \times\{0,1,2\} \times A_{1}^{\perp}$.

- $E^{f}$ contains the following edges:

- For $s_{1}^{f}=\langle\widehat{R}, 1\rangle \in S_{1}^{f}$, the set $E^{f}$ contains the outgoing edges $\left(\langle\widehat{R}, 1\rangle,\left\langle\widehat{R}, j, a_{1}, 2\right\rangle\right)$ such that in the structure $\widehat{\mathcal{T}}_{3 b l}^{\Omega^{\mathrm{td}}}$, there exists $\widehat{s} \in \widehat{R}$ and $\left\langle\Delta, a_{1}\right\rangle \in \Gamma_{1}^{\widehat{\mathcal{T}}_{3 b l}^{\Omega^{\mathrm{td}}}}(\widehat{s})$ with $\left|\left\{\operatorname{Reg}\left(\widehat{s}+\Delta^{\prime}\right) \mid \Delta^{\prime} \leq \Delta\right\}\right|=j+1$. These types of edges encode in $\mathcal{T}^{f}$ the fact that in the game structure $\widehat{\mathcal{T}}_{3 b l}^{\text {td }}$ there exists a state in $\widehat{R}$ such that player 1 has a move such that time passes to the $j$-th following region from where the discrete action $a_{1}$ is taken.

- For $s_{2}^{f}=\left\langle\widehat{R}, j, a_{1}, 2\right\rangle$ in $S_{2}^{f}$, the set $E^{f}$ contains the outgoing edges $\left(\left\langle\widehat{R}, j, a_{1}, 2\right\rangle,\left\langle\widehat{R}^{\prime}, 1\right\rangle\right)$ such that in the structure $\widehat{\mathcal{T}}_{3 b l}^{\Omega^{\mathrm{td}}}$, there exists $\widehat{s} \in \widehat{R},\left\langle\Delta_{1}, a_{1}\right\rangle \in \Gamma_{1}(\widehat{s})$ and $\left\langle\Delta_{2}, a_{2}\right\rangle \in$ $\Gamma_{2}(\widehat{s})$ with $\left|\left\{\operatorname{Reg}\left(\widehat{s}+\Delta^{\prime}\right) \mid \Delta^{\prime} \leq \Delta_{1}\right\}\right|=j+1$ such that either

(1) $a_{1} \neq \perp_{*}, \quad\left|\left\{\operatorname{Reg}\left(\widehat{s}+\Delta^{\prime}\right) \mid \Delta^{\prime} \leq \Delta_{2}\right\}\right| \leq j+1$ and $\delta\left(\widehat{s},\left\langle\Delta_{2}, a_{2}\right\rangle\right) \in \widehat{R}^{\prime}$.

This edge corresponds to the case when player 2 does not allow a player- 1 move $\left\langle\Delta_{1}, a_{1}\right\rangle$ from a state $\widehat{s} \in \widehat{R}$ by proposing its own move $\left\langle\Delta_{2}, a_{2}\right\rangle$ such that $\Delta_{2} \leq \Delta_{1}$.

(2) $a_{1} \neq \perp_{*}, \quad 3 \geq\left|\left\{\operatorname{Reg}\left(\widehat{s}+\Delta^{\prime}\right) \mid \Delta^{\prime} \leq \Delta_{2}\right\}\right| \geq j+1$ and $\delta\left(\widehat{s},\left\langle\Delta_{1}, a_{1}\right\rangle\right) \in \widehat{R}^{\prime}$.

This edge corresponds to the case when player 2 allows a player-1 move $\left\langle\Delta_{1}, a_{1}\right\rangle$ from a state $\widehat{s} \in \widehat{R}$ by proposing a move of duration $\Delta_{2} \geq \Delta_{1}$.

(3) $a_{1}=\perp_{*}$ and $\delta\left(\widehat{s},\left\langle\Delta_{2}, a_{2}\right\rangle\right) \in \widehat{R}^{\prime}$.

This edge corresponds to the case when player 1 plays a $\perp_{*}$ move so the move of player 2 is chosen.

Note that we can pick any states in $\widehat{R}$ and $\widehat{R}^{\prime}$ to check the satisfiability of the above conditions by Lemma 3.1 .

In the construction for $\mathcal{T}_{\Omega}^{f}$ above, the states in $\widehat{S}_{\text {Tup }} \times\{2\}$ contain player- 1 actions as components. The actions are used only for determining the destination states, so instead of player-1 actions as components of states in $\widehat{S}_{\text {Tup }}$ we can as well use a tuple from $L \times 2^{C}$, with $\langle l, \alpha\rangle$ denoting an action in $\mathcal{T}$ such that the destination location is $l$, and which resets the clocks in $\alpha$.

Each $s^{f} \in S^{f}$ is a tuple, with the first component being a region of $\mathcal{T}$. Given the location parity index function $\Omega_{3 b l}^{\mathrm{td}}$ on $\widehat{\mathcal{T}}_{3 b l}^{\Omega^{\mathrm{td}}}$, we let $\Omega^{f}$ be the parity index function on $\mathcal{T}_{\Omega}^{f}$ such that $\Omega^{f}(\langle\widehat{R}, \cdot\rangle)=\Omega_{3 b l}^{\mathrm{td}}(\widehat{s})$ where $\widehat{s}$ is any state in the region $\widehat{R}$ (all states in a region have the same parity as $\Omega_{3 b l}^{\mathrm{td}}$ is a location parity index function on $\widehat{\mathcal{T}}_{3 b l}^{\Omega^{\mathrm{td}}}$ ). Note that if the parity index function $\Omega$ is of order $d$, then $\Omega_{3 b l}^{\text {td }}$ and $\Omega^{f}$ are of order $d+2$. Given a set $X=X_{1} \times\{1\} \cup X_{2} \times\{2\} \subseteq S^{f}$, we let $\operatorname{RegStates}(X)=\left\{\widehat{s} \in S^{\widehat{\mathcal{T}}_{3 b l}^{\text {td }}} \mid \operatorname{Reg}(\widehat{s}) \in X_{1}\right\}$. Theorem 3.10 shows that the turn-based game $\mathcal{T}_{\Omega}^{f}$ captures the game $\widehat{\mathcal{T}}_{3 b l}^{\Omega^{\mathrm{td}}}$. 
Theorem 3.10. Let $\mathcal{T}$ be a timed automaton game, $\Omega$ a location parity index function on states of $\mathcal{T}$, with $\widehat{\mathcal{T}}_{3 b l}^{\Omega^{\mathrm{td}}}$ being the corresponding enlarged game, and $\mathcal{T}^{f}$ the corresponding finite game structure with the parity index function $\Omega^{f}$. Then, we have

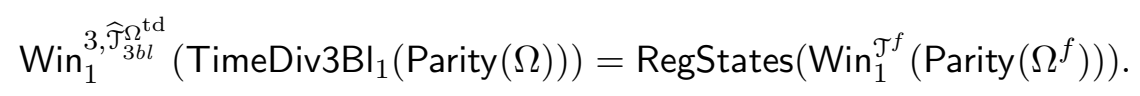

Proof. Recall the $\mu$-calculus formulation for obtaining the winning set

$$
\operatorname{Win}_{1}^{3, \widehat{\mathcal{T}}_{3 b l}^{\Omega^{\mathrm{td}}}\left(\operatorname{TimeDiv}^{\mathrm{B}} \mathrm{BI}_{1}(\operatorname{Parity}(\Omega))\right)}
$$

in $\widehat{\mathcal{T}}_{3 b l}^{\Omega^{\mathrm{td}}}$. We use the Pre ${ }_{1}$ operator in turn-based games:

$$
\operatorname{Pre}_{1}(X)=\begin{aligned}
& \left\{s \in \widehat{S}_{\text {Reg }} \times\{1\} \mid \exists s^{\prime} \in X \text { such that }\left(s, s^{\prime}\right) \in E^{f}\right\} \\
& \left.\cup s \in \widehat{S}_{\text {Tup }} \times\{2\} \mid \forall\left(s, s^{\prime}\right) \in E^{f} \text { we have } s^{\prime} \in X\right\}
\end{aligned}
$$

Given $X=X_{1} \times\{1\} \cup X_{2} \times\{2\} \subseteq S^{f}$, we have

$$
\operatorname{Reg} \text { States }\left(\operatorname{Pre}_{1}^{\mathcal{T}^{f}}\left(\operatorname{Pre}_{1}^{\mathcal{T}^{f}}(X)\right)\right)=\operatorname{RegStates}\left(\operatorname{Pre}_{1}^{\mathcal{T}^{f}}\left(\operatorname{Pre}_{1}^{\mathcal{T}^{f}}\left(X_{1} \times\{1\}\right)\right)\right)
$$

This is because the structure of the games $\mathcal{T}_{\Omega}^{f}$ is such that a state with 1 as the last component of the tuple can reach $X=X_{1} \times\{1\} \cup X_{2} \times\{2\}$ in exactly two steps iff it can reach $X_{1} \times\{1\}$ in exactly two steps (the game is bipartite).

From Lemma 3.1, it follows that

$$
\operatorname{RegStates}\left(\operatorname{Pre}_{1}^{\mathcal{T}^{f}}\left(\operatorname{Pre}_{1}^{\mathcal{T}^{f}}\left(X_{1} \times\{1\}\right)\right)\right)=\mathrm{CPre}_{1}^{\widehat{\widetilde{\tau}}_{3 b l}^{\Omega^{\mathrm{td}}}}\left(\operatorname{Reg} \operatorname{States}\left(X_{1} \times\{1\}\right)\right)
$$

Let $\phi_{c}$ be the $\mu$-calculus formula using the CPre ${ }_{1}$ operator describing the winning set for $\operatorname{Parity}\left(\Omega_{3 b l}^{\text {td }}\right)=\operatorname{TimeDiv}_{3} \mathrm{Bl}_{1}(\operatorname{Parity}(\Omega))$. Let $\phi_{t}$ be the $\mu$-calculus formula using the Pre $_{1}$ operator in a turn-based game describing the winning set for $\operatorname{Parity}\left(\Omega^{f}\right)$. As $\Omega^{f}$ and $\Omega_{3 b l}^{\mathrm{td}}$ contain the same number of priorities, the formula $\phi_{t}$ can be obtained from $\phi_{c}$ by syntactically replacing every $\mathrm{CPre}_{1}$ by $\operatorname{Pre}_{1}$ (see dAHM01). Let the winning set for Parity $\left(\Omega^{f}\right)$ in $\mathcal{T}_{\Omega}^{f}$ be $W_{1} \times\{1\} \cup W_{2} \times\{2\}$. It is described by $\phi_{t}$. The game in $\mathcal{T}^{f}$ proceeds in a bipartite fashion - player 1 and player 2 alternate moves, with the state resulting from the move of player 1 having the same parity index as the originating state. Note that the objective Parity $\left(\Omega^{f}\right)$ depends only on the infinitely often occurring indices in the trace. Thus, $W_{1} \times\{1\}$ can be also be described by the $\mu$-calculus formula $\phi_{t}^{\prime}$ obtained by replacing each Pre ${ }_{1}$ in $\phi_{t}$ with Pre $_{1} \circ$ Pre $_{1}$, and taking states of the form $s \times\{1\}$ in the result. Consider the fixpoint iteration $\mathcal{I}_{\phi_{t}^{\prime}}$ for computing the set $\phi_{t}^{\prime}$. Since we are only interested in the set $W_{1} \times\{1\}$, and since we have a bipartite game, the set $W_{1} \times\{1\}$ can also be described by an iteration $\mathcal{I}^{*}$ in which each set in the iteration $\mathcal{I}_{\phi_{t}^{\prime}}$ is intersected with $\widehat{S}_{\text {Reg }} \times\{1\}$. This is because each step in the iteration $\mathcal{I}_{\phi_{t}^{\prime}}$ applies Pre $_{1} \circ$ Pre $_{1}$, and if $X_{1} \times\{1\} \cup X_{2} \times\{2\}=$ Pre $_{1} \circ \operatorname{Pre}_{1}\left(Z_{1} \times\{1\} \cup Z_{2} \times\{2\}\right)$, then $X_{1} \times\{1\}=\operatorname{Pre}_{1} \circ \operatorname{Pre}_{1}\left(Z_{1} \times\{1\}\right)$. Now, this new iteration $\mathcal{I}^{*}$ describes the winning sets of the $\mu$-calculus formula $\phi_{t}^{\prime \prime}$ obtained from $\phi_{t}^{\prime}$ by intersecting every variable with $\widehat{S}_{\text {Reg }} \times\{1\}$. Using the identity 3.2, we have that the sets in the fixpoint iteration computation of $\phi_{t}^{\prime \prime}$ correspond to the sets in the fixpoint iteration computation of $\phi_{c}$, that is, if $X \times\{1\}$ occurs in the computation of $\phi_{t}^{\prime \prime}$ at stage $j$, then $\operatorname{RegStates}(X)$ occurs in the computation of $\phi_{c}$ at the same stage $j$. This implies that the sets are the same on termination for both $\phi_{t}^{\prime \prime}$ and

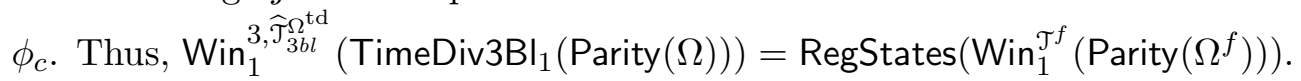


From Theorem 3.10, we can solve the finite-state game $\mathcal{T}^{f}$ to compute winning sets for all $\omega$-regular region parity objectives $\operatorname{Parity}(\Omega)$ for a timed automaton game $\mathcal{T}$, using any algorithm for finite state turn-based games, e.g., strategy improvement, small-progress algorithms [VJ00, Jur00].

3.2.2. Construction of the finite turn-based game $\mathcal{T}_{\Omega}^{f^{*}}$. We now present the turn-based game $\mathcal{T}_{\Omega}^{f}$ ( which can be used to compute the sets $\operatorname{Reg} \operatorname{States}\left(\operatorname{Win}_{1}^{\mathcal{T}_{\Omega}^{f}}\left(\operatorname{Parity}\left(\Omega^{f}\right)\right)\right)$ of $\mathcal{T}_{\Omega}^{f}$, with the state space of $\mathcal{T}_{\Omega}^{f *}$ being linear in the number of regions of $\widehat{\mathcal{T}}_{3 b l}^{\Omega^{\mathrm{td}}}$. The idea behind this construction is that when player 1 proposes a move $\left\langle\Delta, a_{1}\right\rangle$ in $\widehat{\mathcal{T}}_{3 b l}^{\Omega^{\text {td }}}$ from state $\widehat{s}$, player 2 can ignore the discrete component $a_{1}$ of the move. Intuitively this is because player 2 should only care about the moves it can make before time $\Delta$ from state $\widehat{s}$. If there is a move it would like to make before time $\Delta$, then player 2 should preempt the move of player 1 . If there is no such move, then it should allow the move of player 1 , irrespective of $a_{1}$. The exact value of $a_{1}$ (other than whether it is $\perp_{*}$ or not) is irrelevant in determining the moves for which player 2 "wins" in a round. Since the value of $a_{1}$ is irrelevant, there is no need to explicitly remember the discrete component of the moves of player 1 as was done in the game structure $\mathcal{T}_{\Omega}^{f}$. This allows us to bring the state space down, so that it is linear in the number of regions of $\widehat{\mathcal{T}}_{3 b l}^{\Omega^{\text {td }}}$. In the game $\mathcal{T}_{\Omega}^{f}$, we broke a step of the game in $\widehat{\mathcal{T}}_{3 b l}^{\Omega^{\text {td }}}$ into two steps. In the game $\mathcal{T}_{\Omega}^{f *}$, we break a step of $\widehat{\mathcal{T}}_{3 b l}^{\text {td }}$ into three steps: first player 1 makes a move, then, player 2, then player 1 and then the sequence repeats, i.e., the sequence of players making moves is $(121)^{\omega}$. Note that this game is not bipartite as player 1 is making two consecutive moves. Informally, the three steps are as follows.

(1) First, player 1 proposes either a

- Relinquishing move, or

- A move which corresponds to time elapsing to a desired region. The discrete part of the move is left for later.

(2) Then, player 2 moves according to one of the following:

- If player 1 had relinquished in the previous step, then it transitions to a player 1 state such that the region component is the new region. In addition, this is a special player 1 state (we shall see what this means in a while).

- If player 1 had proposed a non-relinquishing move to a region, then player 2 either

- Blocks the player 1 move by proposing a move that corresponds to a shorter duration move in $\widehat{\mathcal{T}}_{3 b l}^{\Omega^{\text {td }}}$. With this move, it transitions to a player 1 state such that the region component is the new region. In addition, this is again a special player 1 state.

- Decides to allow the player 1 move. But remember that we only know the time elapse part of the player 1 move till now. Thus, this move of player 2 transitions the game into an intermediate state from which player 1 can only propose the remaining discrete part of the move.

(3) In the third stage of the game, if the state is not special, then player 1 proposes the remaining discrete part of its original move. If the state is special, then it only has one dummy move, which makes the special state non-special without changing the region component. The special states thus allow us to maintain a three stage game.

Formally, given a timed automaton $\mathcal{T}$, and a location parity index function $\Omega$ on states of $\mathcal{T}$, the finite turn-based game $\mathcal{T}_{\Omega}^{f^{*}}$ consists of a tuple $\left\langle S^{f^{*}}, E^{f^{*}}, S_{1}^{f^{*}}, S_{2}^{f^{*}}\right\rangle$ where, 
- $S^{f^{*}}=S_{1}^{f^{*}} \cup S_{2}^{f^{*}}$ is the state space. The states in $S_{i}^{f^{*}}$ are controlled by player- $i$ for $i \in\{1,2\}$.

- $S_{1}^{f^{*}}=\widehat{S}_{\mathrm{Reg}} \times\left\{1_{a}, 1_{c}^{\text {dum }}\right\} \cup \widehat{S}_{\mathrm{Reg}} \times\{0,1,2\} \times\left\{1_{c}\right\}$, where $\widehat{S}_{\mathrm{Reg}}$ is the set of regions in $\widehat{\mathcal{T}}_{3 b l}^{\Omega^{\mathrm{td}}}$. Informally, the sequence of the players making moves is $(121)^{\omega}$; the moves from states labelled with $1_{a}$ as the last component will correspond to the first "1" and from states labelled with $1_{c}^{\text {dum }}$ or $1_{c}$ as the last component will correspond to the second " 1 " in $(121)^{\omega}$.

- $S_{2}^{f^{*}}=\widehat{S}_{\text {Reg }} \times\{2\} \cup \widehat{S}_{\text {Reg }} \times\{0,1,2\} \times\{2\}$.

- $E^{f^{*}}$ contains the following edges:

- For $s_{1}^{f}=\left\langle\widehat{R}, 1_{a}\right\rangle$, the set $E^{f^{*}}$ contains the following outgoing edges

(1) $\left(\left\langle\widehat{R}, 1_{a}\right\rangle,\langle\widehat{R}, 2\rangle\right)$. These edges correspond to player 1 playing a relinquishing move from states in $\widehat{R}$ in the game structure $\widehat{\mathcal{T}}_{3 b l}^{\Omega^{\mathrm{td}}}$.

(2) $\left(\left\langle\widehat{R}, 1_{a}\right\rangle,\langle\widehat{R}, j, 2\rangle\right)$ such that in the structure $\widehat{\mathcal{T}}_{3 b l}^{\Omega^{\mathrm{td}}}$, there exists $\widehat{s} \in \widehat{R}$ and $\left\langle\Delta, a_{1}\right\rangle \in$ $\Gamma_{1}^{\widehat{\mathcal{T}}_{3 b l}^{\Omega^{\mathrm{td}}}}(\widehat{s})$ with $\left|\left\{\operatorname{Reg}\left(\widehat{s}+\Delta^{\prime}\right) \mid \Delta^{\prime} \leq \Delta\right\}\right|=j+1$. These types of edges encode in $\mathcal{T}^{f^{*}}$ the fact that in the game structure $\widehat{\mathcal{T}}_{3 b l}^{\Omega^{\text {td }}}$ there exists a state in $\widehat{R}$ such that player 1 has a move such that time passes to the $j$-th following region from where the discrete action $a_{1}$ is taken.

- For $s_{2}^{f}=\langle\widehat{R}, 2\rangle$, the set $E^{f^{*}}$ contains the outgoing edges $\left(\langle\widehat{R}, 2\rangle,\left\langle\widehat{R}^{\prime}, 1_{c}^{\text {dum }}\right\rangle\right)$ such that in the structure $\widehat{\mathcal{T}}_{3 b l}^{\Omega^{\mathrm{td}}}$, there exists a state $\widehat{s} \in \widehat{R}$ and $\left\langle\Delta, a_{2}\right\rangle \in \Gamma_{2}^{\widehat{\mathcal{T}}_{3 b l}^{\Omega^{\mathrm{td}}}}(\widehat{s})$ with $\delta\left(\widehat{s},\left\langle\Delta, a_{2}\right\rangle\right) \in \widehat{R}^{\prime}$ and $\operatorname{Succr}_{3}(\widehat{s}, \Delta)=$ TRUE. These edges correspond to the fact in the game structure $\widehat{\mathcal{T}}_{3 b l}^{\Omega^{\mathrm{td}}}$, player 1 had played a relinquishing move from some state $\widehat{s}$ in $\widehat{R}$, and player 2 had played a move according to its 3-region strategy.

- For $s_{2}^{f}=\langle\widehat{R}, j, 2\rangle$, the set $E^{f^{*}}$ contains the following outgoing edges:

(1) $\left(\langle\widehat{R}, j, 2\rangle,\left\langle\widehat{R}^{\prime}, 1_{c}^{\text {dum }}\right\rangle\right)$ such that in the structure $\widehat{\mathcal{T}}_{3 b l}^{\Omega^{\text {td }}}$, there exists a state $\widehat{s} \in \widehat{R}$ and $\left\langle\Delta, a_{2}\right\rangle \in \Gamma_{2}^{\widehat{\tau}_{3 b l}^{\Omega^{\text {td }}}}(\widehat{s})$ with $\delta\left(\widehat{s},\left\langle\Delta, a_{2}\right\rangle\right) \in \widehat{R}^{\prime}$ and $\left|\left\{\operatorname{Reg}\left(\widehat{s}+\Delta^{\prime}\right) \mid \Delta^{\prime} \leq \Delta\right\}\right| \leq j+1$. These types of edges encode in $\mathcal{T}^{f^{*}}$ the fact that in the game structure $\widehat{\mathcal{T}}_{3 b l}^{\Omega^{\text {td }}}$ there exists a state in $\widehat{R}$ such that from $\widehat{s}$ player 1 proposes a move such that time would pass to the $j$-th following region from where some discrete action would be taken, and player 2 is countering this player 1 move with a move of shorter(or equal) delay.

(2) $\left(\langle\widehat{R}, j, 2\rangle,\left\langle\widehat{R}, j, 1_{c}^{\text {dum }}\right\rangle\right)$. This edge encode in $\mathcal{T}^{f^{*}}$ the fact that in the game structure $\widehat{\mathcal{T}}_{3 b l}^{\Omega^{\mathrm{td}}}$ there exists a state in $\widehat{R}$ such that from $\widehat{s}$ player 1 proposes a move such that time would pass to the $j$-th following region from where some discrete action would be taken, and player 2 is "allowing" this move of player 1 .

- For $s_{1}^{f}=\left\langle\widehat{R}, j, 1_{c}\right\rangle$, the set $E^{f^{*}}$ contains the outgoing edges $\left(\left\langle\widehat{R}, j, 1_{c}\right\rangle,\left\langle\widehat{R}^{\prime}, 1\right\rangle\right)$ such that in the structure $\widehat{\mathcal{T}}_{3 b l}^{\Omega^{\mathrm{td}}}$, there exists a state $\widehat{s} \in \widehat{R}$ and $\left\langle\Delta, a_{1}\right\rangle \in \Gamma_{1}^{\widehat{\mathcal{T}}_{3 b l}^{\Omega^{\mathrm{td}}}}(\widehat{s})$ with $\delta\left(\widehat{s},\left\langle\Delta, a_{1}\right\rangle\right) \in \widehat{R}^{\prime}$ and $\left|\left\{\operatorname{Reg}\left(\widehat{s}+\Delta^{\prime}\right) \mid \Delta^{\prime} \leq \Delta\right\}\right|=j+1$. This edge corresponds to the fact that in the game $\widehat{\mathcal{T}}_{3 b l}^{\Omega^{\text {td }}}$ player 2 has allowed player 1 to take a move from a state $\widehat{s}$ in $\widehat{R}$ such that time passes to the $j$-th following region from where the discrete action $a_{1}$ is taken.

- For $s_{1}^{f}=\left\langle\widehat{R}, 1_{c}^{\text {dum }}\right\rangle$, the set $E^{f^{*}}$ contains the single outgoing edge $\left(\left\langle\widehat{R}, 1_{c}^{\text {dum }}\right\rangle,\left\langle\widehat{R}, 1_{a}\right\rangle\right)$. This is just a dummy edge, to preserve the $(121)^{\omega}$ move pattern. 
Given a set $X=X_{1_{a}} \times\left\{1_{a}\right\} \cup X_{1_{c}} \times\left\{1_{c}, 1_{c}^{\text {dum }}\right\} \cup X_{2} \times\{2\} \subseteq S^{f^{*}}$, we let $\operatorname{Reg} \operatorname{States}(X)=$ $\left\{\widehat{s} \in S^{\widehat{\mathcal{T}}_{3 b l}^{\Omega^{\mathrm{td}}}} \mid \operatorname{Reg}(\widehat{s}) \in X_{1_{a}}\right\}$. Given the location parity index function $\Omega_{3 b l}^{\mathrm{td}}$ on $\widehat{\mathcal{T}}_{3 b l}^{\Omega^{\mathrm{td}}}$, we let $\Omega^{f^{*}}$ be the parity index function on $\mathcal{T}^{f}$ such that $\Omega^{f^{*}}(\langle\widehat{R}, \cdot\rangle)=\Omega_{3 b l}^{\mathrm{td}}(\widehat{s})$ where $\widehat{s}$ is any state in the region $\widehat{R}$ (all states in a region have the same parity as $\Omega_{3 b l}^{\mathrm{td}}$ is a location parity index function on $\left.\widehat{\mathcal{T}}_{3 b l}^{\Omega^{\mathrm{td}}}\right)$.

The following lemma shows that two steps in the bipartite game $\mathcal{T}_{\Omega}^{f}$ correspond to three steps in the game $\mathcal{T}_{\Omega}^{f^{*}}$. The proof is presented in the appendix.

Lemma 3.11. Let $\mathcal{T}$ be a timed automaton game, $\Omega$ a location parity index function on states of $\mathcal{T}$, with $\widehat{\mathcal{T}}_{3 b l}^{\Omega^{\mathrm{td}}}$ being the corresponding enlarged game, and $\mathcal{T}^{f}, \mathcal{T}^{f^{*}}$ being the corresponding finite game structures with the parity index functions $\Omega^{f}$ and $\Omega^{f *}$ respectively. Suppose $X \subseteq S^{f}$ and $X^{*} \subseteq S^{f}$ are such that $\operatorname{Reg} \operatorname{States}(X)=\operatorname{Reg} \operatorname{States}\left(X^{*}\right)$. Then, $\operatorname{RegStates}\left(\operatorname{Pre}_{1}^{\mathcal{T}^{f}}\left(\operatorname{Pre}_{1}^{\mathcal{T}^{f}}(X)\right)\right)=\operatorname{Reg} \operatorname{States}\left(\operatorname{Pre}_{1}^{\mathcal{T}^{f^{*}}}\left(\operatorname{Pre}_{1}^{\mathcal{T}^{f^{*}}}\left(\operatorname{Pre}_{1}^{\mathcal{T}^{f^{*}}}\left(X^{*}\right)\right)\right)\right)$.

Using the fact that two steps in the bipartite game $\mathcal{T}_{\Omega}^{f}$ correspond to three steps in the game $\mathcal{T}_{\Omega}^{f *}$, we have the following theorem which shows that we can use the game structure $\mathcal{T}_{\Omega}^{f^{*}}$ to compute the winning sets for $\mathcal{T}$.

Theorem 3.12. Let $\mathcal{T}$ be a timed automaton game, $\Omega$ a location parity index function on states of $\mathcal{T}$, with $\widehat{\mathcal{T}}_{3 b l}^{\Omega^{\mathrm{td}}}$ being the corresponding enlarged game, and $\mathcal{T}^{f}, \mathcal{T}^{f *}$ being the corresponding finite game structures with the parity index functions $\Omega^{f}$ and $\Omega^{f *}$ respectively. Then, we have $\operatorname{Reg} \operatorname{States}\left(\operatorname{Win}_{1}^{\mathcal{T}^{f}}\left(\operatorname{Parity}\left(\Omega^{f}\right)\right)\right)=\operatorname{RegStates}\left(\operatorname{Win}_{1}^{\mathcal{T}^{f *}}\left(\operatorname{Parity}\left(\Omega^{f^{*}}\right)\right)\right)=$

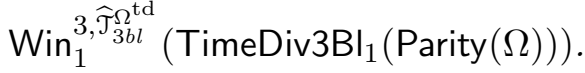

Proof. Let the winning set in $\mathcal{T}_{\Omega}^{f}$ be $W_{1} \times\{1\} \cup W_{2} \times\{2\}$; and the winning set in $\mathcal{T}_{\Omega}^{f *}$ be $W_{1_{a}}{ }^{*} \times\left\{1_{a}\right\} \cup W_{1_{c}}{ }^{*} \times\left\{1_{c}, 1_{c}^{\text {dum }}\right\} \cup W_{2}{ }^{*} \times\{2\}$. As proved in the proof of Theorem 3.10, the set $W_{1} \times\{1\}$ is also described by the $\mu$-calculus formula $\phi_{t}^{\prime \prime}$ obtained from the $\mu$-calculus formula $\phi_{c}$, which describes the winning set for Parity $\left(\Omega_{3 b l}^{\mathrm{td}}\right)$, by (1) syntactically replacing each occurrence of $\mathrm{CPre}_{1}$ in $\phi_{c}$ by $\mathrm{Pre}_{1} \circ \mathrm{Pre}_{1}$, and, (2) intersecting each variable in $\phi_{c}$ with $\widehat{S}_{\text {Reg }}$ (where $\widehat{S}_{\text {Reg }}$ is the set of regions of $\widehat{\mathcal{T}}_{3 b l}^{\Omega^{\mathrm{td}}}$ ). Using a similar argument, the set $W_{1_{a}}{ }^{*} \times\left\{1_{a}\right\}$ can be described by a $\mu$-calculus formula $\phi_{t}^{*}$ obtained from $\phi_{c}$ by (1) syntactically replacing each occurrence of $\mathrm{CPre}_{1}$ in $\phi_{c}$ by Pre $_{1} \circ \mathrm{Pre}_{1} \circ \mathrm{Pre}_{1}$, and, (2) intersecting each variable in $\phi_{c}$ with $\widehat{S}_{\text {Reg. }}$. By Lemma 3.11, we have that the sets in the fixpoint iteration computation of $\phi_{t}^{\prime \prime}$ correspond to the sets in the fixpoint iteration computation of $\phi_{t}^{*}$, that is, if $X \times\{1\}$ occurs in the computation of $\phi_{t}^{\prime \prime}$ at stage $j$, then $X \times\left\{1_{a}\right\}$ occurs in the computation of $\phi_{t}^{*}$ at the same stage $j$. This implies that the sets are the same on termination for both $\phi_{t}^{\prime \prime}$ and $\phi_{t}^{*}$. Thus, $W_{1}=W_{1_{a}}{ }^{*}$, and hence $\operatorname{Reg} \operatorname{States}\left(\operatorname{Win}_{1}^{\mathcal{T}^{f}}\left(\operatorname{Parity}\left(\Omega^{f}\right)\right)\right)=\operatorname{Reg} \operatorname{States}\left(\operatorname{Win}_{1}^{\mathcal{T}^{f *}}\left(\operatorname{Parity}\left(\Omega^{f^{*}}\right)\right)\right)=$

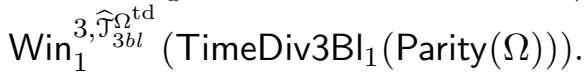

Eliminating redundant states in $\mathcal{T}^{\mathbf{f}}$. The states with $1_{c}^{\text {dum }}$ as the last component are redundant and were introduced just to have a three stage game to prove Theorem 3.12 using Lemma 3.11. These redundant states can be removed as follows. The objective Parity $\left(\Omega^{f^{*}}\right)$ depends only on the infinitely often occurring indices in traces. Consider the states in $\mathcal{T}^{f *}$ of the form $\left\langle\widehat{R}, 1_{c}^{\text {dum }}\right\rangle$. Player 1 has only one move from such a state, to $\left\langle\widehat{R}, 1_{a}\right\rangle$. The parity of $\left\langle\widehat{R}, 1_{c}^{\text {dum }}\right\rangle$ is the same as the parity of $\left\langle\widehat{R}, 1_{a}\right\rangle$. Thus, these kinds of states can be removed, 
and the incoming edges made to point to the destination of these states without changing the part of the winning set with $1_{a}$ as the last component. That is, given a state $\left\langle\widehat{R}, 1_{c}^{\text {dum }}\right\rangle$ with $m$ incoming edges $\left(s^{f^{*}},\left\langle\widehat{R}, 1_{c}^{\text {dum }}\right\rangle\right)$ for $1 \leq j \leq m$, we can remove the state $\left\langle\widehat{R}, 1_{c}^{\text {dum }}\right\rangle$ and add $m$ edges $\left(s^{f^{*}},\left\langle\widehat{R}, 1_{a}\right\rangle\right)$ for $1 \leq j \leq m$ without changing the part of the winning set with $1_{a}$ as the last component.

3.2.3. Complexity of reduction. Recall that for a timed automaton game $\mathcal{T}, A_{i}$ is the set of actions for player $i$ and $C$ is the set of clocks. Let $\left|A_{1}\right|^{*}=1+\min \left\{\left|A_{1}\right|+1,|L| \cdot 2^{|C|}\right\}$ (the +1 outside is due to the relinquishing move of player 1 , the min is because we can either use player-1 actions, including pure time moves; or use tuples of locations with clock reset sets as actions), and let $\left|A_{2}\right|^{*}=\min \left\{\left|A_{2}\right|+1,|L| \cdot 2^{|C|}\right\}$. Let $\left|\mathcal{T}_{\text {Constr }}\right|$ denote the length of the clock constraints in $\mathcal{T}$ and $d$ be the order of the parity index function $\Omega$. The size of the state space of $\mathcal{T}^{f^{*}}$ (with the $1_{c}^{\text {dum }}$ states eliminated) is bounded by $8 \cdot\left|S_{\operatorname{Reg}}^{\widehat{\mathcal{T}}_{3 b l}^{\Omega^{\text {td }}}}\right|$ where $S_{\operatorname{Reg}}^{\widehat{\mathcal{T}}_{3 b l}^{\Omega^{\text {td }}}}$ is the set of regions of $\widehat{\mathcal{T}}_{3 b l}^{\Omega^{\text {td }}}$. The number of edges originating from player-1 states is at most $\left|S_{\text {Reg }}^{\widehat{\widetilde{T}}^{\mathrm{td}}}\right| \cdot\left(4+3\left|A_{1}\right|^{*}\right)$. The number of edges originating from player-2 states is at most $\left|S_{\operatorname{Reg}}^{\widehat{\widetilde{\Omega}}^{\mathrm{td}}}\right| \cdot 6 \cdot\left|A_{2}\right|^{*}$. The total number of edges is thus at most $\left|S_{\operatorname{Reg}}^{\widehat{\widetilde{T}}^{\Omega^{\mathrm{td}}}}\right| \cdot\left(4+3\left|A_{1}\right|^{*}+6\left|A_{2}\right|^{*}\right)$. We also have $\left|S_{\operatorname{Reg}}^{\widehat{\widetilde{T}}^{\mathrm{td}}}\right|$ to be bounded by $32 \cdot d \cdot|L| \cdot \prod_{x \in C}\left(c_{x}+1\right) \cdot|C+1| ! \cdot 4^{|C|}$.

Theorem 3.13. Let $\mathcal{T}$ be a timed automaton game, and let $\Omega$ be a location parity index function of order $d$. The set WinTimeDiv $\mathcal{T}_{1}^{\mathcal{T}}(\operatorname{Parity}(\Omega))$ can be computed in time

$$
O\left(\left|S_{\text {Reg }}^{\widehat{\widetilde{T}}_{3 b l}^{\text {td }}}\right| \cdot\left|\mathcal{T}_{\text {Constr }}\right|+\left[\left(\left|A_{1}\right|^{*}+\left|A_{2}\right|^{*}\right) \cdot\left(8 \cdot\left|S_{\text {Reg }}^{\widehat{\Im}_{3 b l}^{\Omega^{\mathrm{td}}}}\right|\right)^{\frac{d+2}{3}+\frac{3}{2}}\right]\right)
$$

which equals

$$
O\left(\left|S_{\mathrm{Reg}}^{\mathcal{T}}\right| \cdot d \cdot|C| \cdot\left|\mathcal{T}_{\text {Constr }}\right|+\left[\left(\left|A_{1}\right|^{*}+\left|A_{2}\right|^{*}\right) \cdot\left(\left|S_{\mathrm{Reg}}^{\mathcal{T}}\right| \cdot d \cdot 256 \cdot|C|\right)^{\frac{d+2}{3}+\frac{3}{2}}\right]\right)
$$

where

- $S_{\operatorname{Reg}}^{\mathcal{T}}$ is the set of regions of $\mathcal{T}$, with $\left|S_{\operatorname{Reg}}^{\mathcal{T}}\right|=|L| \cdot \prod_{x \in C}\left(c_{x}+1\right) \cdot|C| ! \cdot 4^{|C|}$,

- $S_{\mathrm{Reg}}^{\widehat{\mathcal{T}}_{3 b l}^{\Omega^{\mathrm{td}}}}$ is the set of regions of $\widehat{\mathcal{T}}_{3 b l}^{\Omega^{\mathrm{td}}}$, with $\left|S_{\mathrm{Reg}}^{\widehat{\mathcal{T}}_{3 b l}^{\Omega^{\mathrm{td}}}}\right|=32 \cdot(|C|+1) \cdot d \cdot\left|S_{\mathrm{Reg}}^{\mathcal{T}}\right|$,

- $\left|\mathcal{T}_{\text {Constr }}\right|$ is the length of the clock constraints in $\mathcal{T}$,

- $\left|A_{1}\right|^{*}=1+\min \left\{\left|A_{1}\right|+1,|L| \cdot 2^{|C|}\right\}$ and $\left|A_{2}\right|^{*}=\min \left\{\left|A_{2}\right|+1,|L| \cdot 2^{|C|}\right\}$ with $A_{i}$ being the set of discrete actions of player $i$ for $i \in\{1,2\}$,

- $L$ is the set of locations, and $C$ is the set of clocks in $\mathcal{T}$.

Proof. To solve for WinTimeDiv ${ }_{1}^{\mathcal{T}}(\operatorname{Parity}(\Omega))$, we solve the turn-based game $A^{f}$ by Theorems 3.9 and 3.12 , and Lemma 3.3. For constructing $\mathcal{T}^{f^{*}}$, we need to check which regions satisfy clock constraints from $\mathcal{T}$. For this, we build a list of regions with valid invariants together with edge constraints satisfied at the region. This takes $O\left(\left|S_{\text {Reg }}^{\widehat{\mathcal{T}}_{3 b l}^{\Omega^{\text {td }}}}\right| \cdot\left|\mathcal{T}_{\text {Constr }}\right|\right)$ time (we assume a region can be represented in constant space in our analysis). From [Sch07], we have that a turn-based parity game with $m$ edges, $n$ states and $d$ parity indices can be 
solved in $O\left(m \cdot n^{\frac{d}{3}+\frac{1}{2}}\right)$ time. The turn-based game $A^{f}$ can hence be solved in time

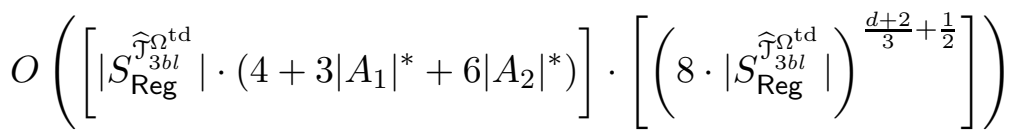

which is equal to

$$
O\left(\left(\left|A_{1}\right|^{*}+\left|A_{2}\right|^{*}\right) \cdot\left[\left(8 \cdot\left|S_{\operatorname{Reg}}^{\widehat{\tau}_{3 b l}^{\Omega \mathrm{td}}}\right|\right)^{\frac{d+2}{3}+\frac{3}{2}}\right]\right)
$$

Remark 3.14 (Effects of receptive formulation). In the complexity, we multiply $\left|S_{\text {Reg }}^{\mathcal{T}}\right|$ by $d$ as we go from Parity $(\Omega)$ to $\operatorname{Parity}\left(\Omega^{\text {td }}\right)=\operatorname{TimeDivBl}_{1}(\operatorname{Parity}(\Omega)$ ) (this also increases the value of the exponent from $\frac{d}{3}+\frac{3}{2}$ to $\left.\frac{d+2}{3}+\frac{3}{2}\right)$. Multiplication of $\left|S_{\text {Reg }}^{\mathcal{T}}\right|$ by $32 \cdot|C|$ comes in due to the extra clock $z$ which measures global time, and introduction of the variables tick and $3 b l_{1}$.

Usefulness of the finite turn-based game $\mathcal{T}^{\mathbf{f}}$. The reduction allows us to solve the finite-state game $\mathcal{T}^{f^{*}}$ to compute winning sets for all $\omega$-regular region parity objectives Parity $(\Omega)$ for a timed automaton game $\mathcal{T}$, using any algorithm for finite state turn-based games, e.g., strategy improvement, small-progress algorithms [VJ00, Jur00. It also allows us to leverage any future improvements in algorithms for finite-state games for solving timed parity games. The reduction to a finite-state game that is linear in the number of regions of $\widehat{\mathcal{T}}_{3 b l}^{\Omega \text { td }}$ also shows that we do not pay any complexity penalty due to the concurrent nature of the timed game where both players simultaneously propose moves.

\section{Robust Winning of Timed Parity Games}

In this section we study restrictions on player-1 strategies to model robust winning, and show how the winning sets can be obtained by reductions to general timed automaton games. The results of Section 3 can then be used to obtain algorithms for computing the robust winning sets.

There is inherent uncertainty in real-time systems. In a physical system, an action may be prescribed by a controller, but the controller can never prescribe a single timepoint where that action will be taken with probability 1 . There is usually some jitter when the specified action is taken, the jitter being non-deterministic. The model of general timed automaton games, where player 1 can specify exact moves of the form $\left\langle\Delta, a_{1}\right\rangle$ consisting of an action together with a precise delay $\Delta$, assumes that the jitter is 0 . In subsection 4.1, we obtain robust winning sets for player 1 in the presence of non-zero jitter (which are assumed to be arbitrarily small) for each of its proposed moves. In subsection 4.2, we assume the jitter to be some fixed $\varepsilon_{\mathrm{j}} \geq 0$ for every move that is known. The strategies of player 2 are left unrestricted. In the case of lower-bounded jitter, we also introduce a response time for player-1 strategies. The response time is the minimum delay between a discrete action, and a discrete action of the controller. We note that the set of moves with a jitter of $\varepsilon_{\mathrm{j}}>0$ around $\left\langle\Delta, a_{1}\right\rangle$ contains the set of moves with a jitter of $\varepsilon_{\mathrm{j}} / 2$ and a response time of $\varepsilon_{\mathrm{j}} / 2$ around $\left\langle\Delta, a_{1}\right\rangle$ (as $\left\{\left\langle\Delta^{\prime}, a_{1}\right\rangle \mid \Delta+\varepsilon_{\mathrm{j}} / 2 \leq \Delta^{\prime} \leq \Delta+\varepsilon_{\mathrm{j}}\right\} \subseteq\left\{\left\langle\Delta^{\prime}, a_{1}\right\rangle \mid \Delta \leq \Delta^{\prime} \leq \Delta+\varepsilon_{\mathrm{j}}\right\}$. Thus, the strategies of subsection 4.1 may be considered to have a response time greater than 0 . The winning sets in both sections are hence robust towards the presence of jitter and response times. 
4.1. Winning in the Presence of Jitter. In this subsection, we model games where the jitter is assumed to be greater than 0 , but arbitrarily small in each round of the game.

Given a state $s$, a limit-robust move for player 1 is either the move $\left\langle\Delta, \perp_{*}\right\rangle$; or it is a tuple $\left\langle[\alpha, \beta], a_{1}\right\rangle$ for some $0 \leq \alpha<\beta$ such that for every $\Delta \in[\alpha, \beta]$ we have $\left\langle\Delta, a_{1}\right\rangle \in \Gamma_{1}(s)$ $2(|\beta-\alpha|$ does not have a lower bound, it just needs to be greater than 0 - hence the term limit-robust). Note that a move $\left\langle\Delta, \perp_{*}\right\rangle$ for player 1 implies that it is relinquishing the current round to player 2 , as the move of player 2 will always be chosen, and hence we allow a singleton time move. Given a limit-robust move $m r o b_{1}$ for player 1 , and a move $m_{2}$ for player 2 , the set of possible outcomes is the set

$$
\left\{\delta_{\text {jd }}\left(s, m_{1}, m_{2}\right) \mid \text { either } \begin{array}{l}
\text { (a) } m r o b_{1}=\left\langle\Delta, \perp_{*}\right\rangle \text { and } m_{1}=m r o b_{1} ; \text { or } \\
\text { (b) } m r o b_{1}=\left\langle[\alpha, \beta], a_{1}\right\rangle \text { and } m_{1}=\left\langle\Delta, a_{1}\right\rangle \text { with } \Delta \in[\alpha, \beta]
\end{array}\right\} .
$$

A limit-robust strategy $\pi_{1}^{\text {rob }}$ for player 1 prescribes limit-robust moves to finite run prefixes. A limit-robust strategy $\pi_{1}^{\text {rob }}$ is receptive if for all player-2 strategies $\pi_{2}$, all states $s \in S$, and all runs $r \in \operatorname{Outcomes}\left(s, \pi_{1}^{\text {rob }}, \pi_{2}\right)$, either $r \in$ Timediv or $r \in$ Blameless $_{i}$. We let $\Pi_{1}^{\text {rob }}$ denote the set of limit-robust strategies for player- 1 and $\Pi_{1}^{\text {rob, } R}$ the set of limit-robust receptive strategies. Given an objective $\Phi$, let RobWinTimeDiv ${ }_{1}^{\mathcal{T}}(\Phi)$ denote the set of states $s$ in $\mathcal{T}$ such that player 1 has a limit-robust receptive strategy $\pi_{1}^{\text {rob }} \in \Pi_{1}^{\text {rob, } R}$ such that for all receptive strategies $\pi_{2} \in \Pi_{2}^{R}$, we have Outcomes $\left(s, \pi_{1}^{\text {rob }}, \pi_{2}\right) \subseteq \Phi$. We say a limit-robust strategy $\pi_{1}^{\text {rob }}$ is region equivalent to a (non-robust) strategy $\pi_{1}$ if for all runs $r$ and for all $k \geq 0$, the following conditions hold:

(i) if $\pi_{1}(r[0 . . k])=\left\langle\Delta, \perp_{*}\right\rangle$, then $\pi_{1}^{\text {rob }}(r[0 . . k])=\left\langle\Delta^{\prime}, \perp_{*}\right\rangle$ with $\operatorname{Reg}(r[k]+\Delta)=\operatorname{Reg}(r[k]+$ $\left.\Delta^{\prime}\right)$; and

(ii) if $\pi_{1}(r[0 . . k])=\left\langle\Delta, a_{1}\right\rangle$ with $a_{1} \neq \perp_{*}$, then $\pi_{1}^{\text {rob }}(r[0 . . k])=\left\langle[\alpha, \beta], a_{1}\right\rangle$ with $\operatorname{Reg}(r[k]+$ $\Delta)=\operatorname{Reg}\left(r[k]+\Delta^{\prime}\right)$ for all $\Delta^{\prime} \in[\alpha, \beta]$.

Note that for any limit-robust move $\left\langle[\alpha, \beta], a_{1}\right\rangle$ with $a_{1} \neq \perp_{*}$ from a state $s$, we must have that the set $\{s+\Delta \mid \Delta \in[\alpha, \beta]\}$ contains an open region of $\mathcal{T}$.

We first present an extension to Lemma 2.7.

Lemma 4.1. Let $\mathcal{T}$ be a timed automaton game and $\widehat{\mathcal{T}}$ be the corresponding enlarged game structure. Let $\widehat{\Phi}$ be an $\omega$-regular region objective of $\widehat{\mathcal{T}}$. If $\pi_{1}$ is a region strategy that is winning for $\widehat{\Phi}$ from $\operatorname{Win}_{1} \widehat{\widetilde{\Phi}}(\widehat{\Phi})$ and $\pi_{1}^{\text {rob }}$ is a robust strategy that is region-equivalent to $\pi_{1}$, then $\pi_{1}^{\text {rob }}$ is a winning strategy for $\widehat{\Phi}$ from $\operatorname{Win}_{1}^{\widehat{\mathcal{T}}}(\widehat{\Phi})$.

Proof. Consider any strategy $\pi_{2}$ for player 2 , and a state $\widehat{s} \in \operatorname{Win}_{1}^{\widehat{\widehat{T}}}(\widehat{\Phi})$. We observe that the set Outcomes $\left(s, \pi_{1}^{\text {rob }}, \pi_{2}\right)$ consists of runs $r$ such that for all $k \geq 0$, either

(1) $\pi_{1}^{\mathrm{rob}}(r[0 . . k])=\left\langle\Delta, \perp_{1}\right\rangle$ and $r[k+1] \in \widehat{\delta}_{\mathrm{jd}}\left(r[k],\left\langle\Delta, \perp_{1}\right\rangle, \pi_{2}(r[0 . . k])\right)$; or

(2) $\pi_{1}^{\text {rob }}(r[0 . . k])=\left\langle[\alpha, \beta], a_{1}\right\rangle$ for some $\beta>\alpha \geq 0$

with $r[k+1] \in \widehat{\delta}_{\mathrm{jd}}\left(r[k],\left\langle\Delta, a_{1}\right\rangle, \pi_{2}(r[0 . . k])\right)$ for some $\Delta \in[\alpha, \beta]$.

It can be observed that

$$
\operatorname{Outcomes}\left(s, \pi_{1}^{\text {rob }}, \pi_{2}\right)=\bigcup_{\pi_{1}^{\prime}} \operatorname{Outcomes}\left(\widehat{s}, \pi_{1}^{\prime}, \pi_{2}\right)
$$

\footnotetext{
${ }^{2}$ We can alternatively have an open, or half-open time interval, the results do not change.
} 
where $\pi_{1}^{\prime}$ ranges over all those (non-robust) player-1 strategies such that for runs $r \in$ Outcomes $\left(\widehat{s}, \pi_{1}^{\prime}, \pi_{2}\right)$ and for all $k \geq 0$ we have

$$
\pi_{1}^{\prime}(r[0 . . k])=\left\{\begin{array}{lll}
\left\langle\Delta, \perp_{1}\right\rangle & \text { if } & \pi_{1}^{\text {rob }}(r[0 . . k])=\left\langle\Delta, \perp_{1}\right\rangle \\
\left\langle\Delta, a_{1}\right\rangle & \text { if } & \pi_{1}^{\text {rob }}(r[0 . . k])=\left\langle[\alpha, \beta], a_{1}\right\rangle
\end{array}\right.
$$

for some $\Delta \in[\alpha, \beta]$; and $\pi_{1}^{\prime}$ acts like $\pi_{1}$ otherwise (note that the runs $r$ and the strategies $\pi_{1}^{\prime}$ are defined inductively with respect to $k$, with $\left.r[0]=\widehat{s}\right)$. Each player-1 strategy $\pi_{1}^{\prime}$ in the preceding union is region equivalent to $\pi_{1}$ since $\pi_{1}^{\text {rob }}$ is region equivalent to $\pi_{1}$ and hence each $\pi_{1}^{\prime}$ is a winning strategy for player 1 by Lemma 2.7. Thus, Outcomes $\left(s, \pi_{1}^{\text {rob }}, \pi_{2}\right)=$ $\bigcup_{\pi_{1}^{\prime}}$ Outcomes $\left(\widehat{s}, \pi_{1}^{\prime}, \pi_{2}\right)$ is a subset of $\widehat{\Phi}$, and hence $\pi_{1}^{\text {rob }}$ is a winning strategy for player 1 .

We now show how to compute the set $\operatorname{RobWinTimeDiv}_{1}^{\mathcal{T}}(\Phi)$. Given a timed automaton game $\mathcal{T}$, we have the corresponding enlarged game structure $\widehat{\mathcal{T}}_{3}$ which encodes timedivergence (we use the modified blame variable $b l_{1,3}$ as in Lemma 3.3). We add another boolean variable to $\widehat{\mathcal{T}}_{3}$ to obtain another game structure $\widehat{\mathcal{T}}_{\text {rob }}$. The state space of $\widehat{\mathcal{T}}_{\text {rob }}$ is $S^{\widehat{\mathcal{T}}} \times\{$ TRUE, FALSE $\}$. The transition relation $\delta^{\widehat{\mathrm{T}}_{\text {rob }}}$ is such that

$$
\delta^{\widehat{\mathcal{T}}_{\text {rob }}}\left(\left\langle\widehat{s}, r b_{1}\right\rangle,\left\langle\Delta, a_{i}\right\rangle\right)=\left\langle\widehat{\delta}\left(\widehat{s},\left\langle\Delta, a_{i}\right\rangle\right), r b_{1}^{\prime}\right\rangle
$$

where $r b_{1}^{\prime}=$ TRUE iff $r b_{1}=$ TRUE and one of the following hold:

(i) $a_{i} \in A_{2}^{\perp}$; or

(ii) $a_{i}=\perp_{*}$; or

(iii) $a_{i} \in A_{1}^{\perp_{1}}$ and $s+\Delta$ belongs to an open region of $\widehat{\mathcal{T}}$.

A region $\widehat{R}$ of $\widehat{\mathcal{T}}$ is said to be open if for all states $\widehat{s} \in \widehat{R}$ we have all the clock values in $\widehat{s}$ to be non-integral. Given a location parity index function $\Omega$ on $\mathcal{T}$ of order $d$, we define another game structure $\widehat{\mathcal{T}}^{\Omega_{\text {rob }}^{\text {td }}}$ (based on $\widehat{\mathcal{T}}_{\text {rob }}$ ) with the parity index function $\Omega_{\text {rob }}^{\text {td }}$ encoding $\operatorname{TimeDivBl}_{1,3}(\operatorname{Parity}(\Omega)) \wedge \square\left(r b_{1}=\right.$ TRUE $)$ as follows. The state space of $\widehat{\mathcal{T}}^{\Omega_{\text {rob }}^{\text {td }}}$ is $S^{\widehat{\mathcal{T}}^{\Omega_{\text {rob }}^{\text {td }}}=}$ $S^{\widehat{\widetilde{T}}_{\text {rob }}} \times\{0, \ldots, d-1\}$. The transition relation $\delta^{\widehat{\widetilde{T}}^{\Omega_{\text {rob }}^{\text {td }}}}$ is specified as follows (similar to $\widehat{\mathcal{T}}^{\Omega^{\text {td }}}$ ). For $\left\langle\Delta, a_{i}\right\rangle \in \Gamma_{i}^{\mathcal{T}}(s)$, we have $\delta^{\widehat{\widetilde{J}}_{\mathrm{rob}}^{\mathrm{td}}}\left(\left\langle s, \mathfrak{z}, t i c k, b l_{1}, r b_{1}, p\right\rangle,\left\langle\Delta, a_{i}\right\rangle\right)=\left\langle s^{\prime}, \mathfrak{z}^{\prime}, t i c k^{\prime}, b l_{1}^{\prime}, r b_{1}^{\prime}, p^{\prime}\right\rangle$ where

- $s^{\prime}=\delta^{\mathcal{T}}\left(s,\left\langle\Delta, a_{i}\right\rangle\right)$.

- $\mathfrak{z}^{\prime}=(\mathfrak{z}+\Delta) \bmod 1$.

- tick $^{\prime}=$ TRUE iff $\mathfrak{z}+\Delta \geq 1$.

- $b l_{1}^{\prime}=$ TRUE iff $i=1$ (i.e., its a player-1 move).

- $r b_{1}^{\prime}= \begin{cases}\text { TRUE } & \text { if } r b_{1}=\text { TRUE, } a_{i} \in A_{1} \cup\left\{\perp_{1}\right\} \\ & \text { and all clock values in } C \cup\{z\} \text { are non-integral } \\ \text { TRUE } & \text { if } r b_{1}=\text { TRUE and } a_{i} \in A_{2}^{\perp} \cup\left\{\perp_{*}\right\} \\ \text { FALSE } & \text { otherwise }\end{cases}$

- $p^{\prime}= \begin{cases}\max \left(p, \Omega\left(s^{\prime}\right)\right) & \text { if tick }=\text { FALSE } \\ \Omega\left(s^{\prime}\right) & \text { if } \text { tick }=\text { TRUE }\end{cases}$ 
The parity index function $\Omega_{\text {rob }}^{\text {td }}$ is defined as

$$
\Omega_{\mathrm{rob}}^{\mathrm{td}}\left(\left\langle s, \mathfrak{z}, t i c k, b l_{1,3}, r b_{1}, p\right\rangle\right)= \begin{cases}1 & \text { if } r b_{1}=\text { FALSE } \\ 0 & \text { if } r b_{1}=\text { TRUE and } t i c k=b l_{1,3}=\text { FALSE } \\ 1 & \text { if } r b_{1}=\text { TRUE and } t i c k=\text { FALSE }, b l_{1,3}=\text { TRUE } \\ p+2 & \text { if } r b_{1}=\text { TRUE and } t i c k=\text { TRUE }\end{cases}
$$

Lemma 4.2. Let $\mathcal{T}$ be a timed automaton game, Parity $(\Omega)$ an objective on $\mathcal{T}$, and $\widehat{\mathcal{T}}_{\mathrm{rob}}^{\mathrm{td}}$ the corresponding enlarged game structure with the parity index function $\Omega_{\mathrm{rob}}^{\mathrm{td}}$. Then in the

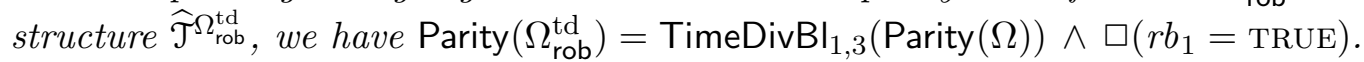

Proof. The proof follows along similar lines to the proof of Lemma 2.5. We also observe than once $r b_{1}$ becomes false, it stays false in $\widehat{\mathcal{T}} \Omega_{\text {rob }}^{\text {td }}$ and hence the parity also stays odd for all the following states. Thus, if the maximum of $\operatorname{InfOften}\left(\Omega_{\mathrm{rob}}^{\mathrm{td}}(\widehat{r})\right)$ is even for a run $\widehat{r}$, then we must have $\square\left(r b_{1}=\right.$ TRUE $)$ in the run.

Theorem 4.3. Given a state $s$ in a timed automaton game $\mathcal{T}$ and an $\omega$-regular location parity index function $\Omega$, we have $s \in \operatorname{RobWinTimeDiv}_{1}^{\mathcal{T}}(\operatorname{Parity}(\Omega))$ iff

$\left\langle s, \cdot, \cdot, \cdot, r b_{1}=\right.$ TRUE, $\left.\cdot\right\rangle \in \operatorname{Win}_{1}^{\widehat{\widetilde{T}}_{\text {rob }}^{\text {td }}}\left(\operatorname{TimeDivBI}_{1,3}(\operatorname{Parity}(\Omega)) \wedge \square\left(r b_{1}=\right.\right.$ TRUE $\left.)\right)$.

Proof.

$\Rightarrow$ Suppose player-1 has a winning limit-robust receptive strategy $\pi_{1}$ for Parity $(\Omega)$, starting from a state $s$ in $\mathcal{T}$. We show

$$
\left\langle s, \cdot, \cdot, \cdot, r b_{1}=\text { TRUE, } \cdot\right\rangle \in \operatorname{Win}_{1}^{\widehat{\widetilde{T}}_{\text {rob }}^{\mathrm{td}}}\left(\operatorname{TimeDivBl}_{1,3}(\operatorname{Parity}(\Omega)) \wedge \square\left(r b_{1}=\text { TRUE }\right)\right) .
$$

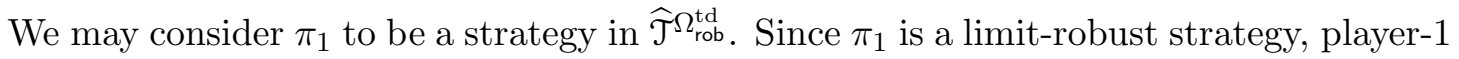
proposes limit-robust moves at each step of the game. Given a state $\widehat{s}$, and a limit-robust move $\left\langle[\alpha, \beta], a_{1}\right\rangle$, there always exists $\alpha<\alpha^{\prime}<\beta^{\prime}<\beta$ such that for every $\Delta \in\left[\alpha^{\prime}, \beta^{\prime}\right]$, we have $\widehat{s}+\Delta$ belonging to an open region of $\widehat{\mathcal{T}}$. Thus, given the limit-robust strategy $\pi_{1}$, we can obtain another limit-robust strategy $\pi_{1}^{\prime}$ in $\widehat{\mathcal{T}}$, such that for every run $\widehat{r}$ and every $k \geq 0$,

(a) if $\pi_{1}(\widehat{r}[0 . . k])=\left\langle\Delta, \perp_{*}\right\rangle$, then $\pi_{1}^{\prime}(\widehat{r}[0 . . k])=\pi_{1}(\widehat{r}[0 . . k])$; and

(b) if $\pi_{1}(\widehat{r}[0 . . k])=\left\langle[\alpha, \beta], a_{1}\right\rangle$, then $\pi_{1}^{\prime}(\widehat{r}[0 . . k])=\left\langle\left(\left[\alpha^{\prime}, \beta^{\prime}\right], a_{1}\right\rangle\right.$ with $\left[\alpha^{\prime}, \beta^{\prime}\right] \subseteq[\alpha, \beta]$, and $\left\{\widehat{r}[k]+\Delta^{\prime} \mid \Delta^{\prime} \in\left[\alpha^{\prime}, \beta^{\prime}\right]\right\}$ being a subset of an open region of $\widehat{\mathcal{T}}^{\Omega_{\text {rob }}^{\text {td }}}$.

Thus for any player-2 strategy $\pi_{2}$, and for any $\operatorname{run} \widehat{r} \in \operatorname{Outcomes}\left(\langle s, \cdot, \cdot, \cdot\right.$, TRUE,,$\left.\cdot\rangle, \pi_{1}^{\prime}, \pi_{2}\right)$, we have that $\widehat{r}$ satisfies $\square\left(r b_{1}=\right.$ TRUE $)$. Since $\pi_{1}$ was a receptive winning strategy for $\operatorname{Parity}(\Omega), \pi_{1}^{\prime}$ is also a receptive winning strategy for Parity $(\Omega)$ as Outcomes $\left(\widehat{s}, \pi_{1}, \pi_{2}\right) \subseteq$ Outcomes $\left(\widehat{s}, \pi_{1}^{\prime}, \pi_{2}\right)$ for any player-2 strategy $\pi_{2}$. Thus, $\pi_{1}^{\prime}$ enables player-1 to satisfy $\operatorname{TimeDivBl}_{1,3}(\operatorname{Parity}(\Omega)) \wedge \square\left(r b_{1}=\right.$ TRUE $)$.

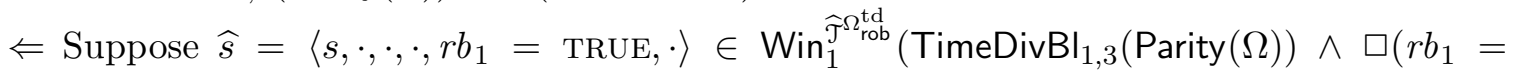
TRUE)). We show that player-1 has a limit-robust receptive winning strategy from state $\widehat{s}$ (and hence from $s$ ). Let $\pi_{1}$ be a winning region strategy for player-1 for the

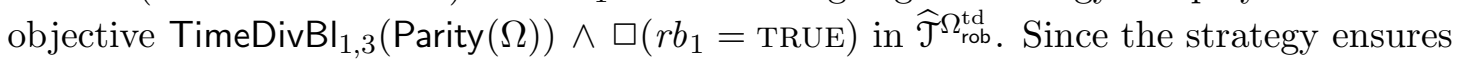
$r b_{1}=$ TRUE for all states in all runs from $\widehat{s}$, we have that for every run $\widehat{r}$ starting from state $\widehat{s}$, the strategy $\pi_{1}$ is such that $\pi_{1}(\widehat{r}[0 . . k])=\left\langle\Delta^{k}, a_{1}^{k}\right\rangle$ where either $a_{1}^{k}=\perp_{*}$, or $\widehat{r}[k]+\Delta^{k}$ belongs to an open region $\widehat{R}$ of $\widehat{\mathcal{T}}^{\Omega_{\text {rob }}^{\text {td }}}$ Since $\widehat{R}$ is an open region, there always exists some $\alpha<\beta$ such that for every $\Delta \in[\alpha, \beta]$, we have $\widehat{r}[k]+\Delta \in \widehat{R}$. Consider 
the strategy $\pi_{1}^{\text {rob }}$ that prescribes a limit-robust move $\left\langle[\alpha, \beta], a_{1}^{k}\right\rangle$ for the history $r[0 . . k]$ if $\pi_{1}(r[0 . . k])=\left\langle\Delta^{k}, a_{1}^{k}\right\rangle$ with $a_{1}^{k} \neq \perp_{*}$, and $\pi_{1}^{\text {rob }}(r[0 . . k])=\pi_{1}(r[0 . . k])$ otherwise. The strategy $\pi_{1}^{\text {rob }}$ is region-equivalent to $\pi_{1}$, and hence is also winning for player-1 by Lemma 4.1 . Since it only prescribes limit-robust moves, it is a limit-robust strategy. It is also a receptive strategy as it is region equivalent to the receptive strategy $\pi_{1}$.

Theorem 4.4. Let $\mathcal{T}$ be a timed automaton game, and let $\Omega$ be a location parity index function of order $d$. The limit-robust winning set $\operatorname{RobWinTimeDiv}_{1}^{\mathcal{T}}(\operatorname{Parity}(\Omega))$ ) can be computed in time

$$
O\left(\left|S_{\mathrm{Reg}}^{\widehat{\widetilde{\tau}}_{\mathrm{rob}}^{\mathrm{td}}}\right| \cdot\left|\mathcal{T}_{\text {Constr }}\right|+\left(\left|A_{1}\right|^{*}+\left|A_{2}\right|^{*}\right) \cdot\left(8 \cdot\left|S_{\mathrm{Reg}}^{\widehat{\widetilde{\tau}}_{\text {rob }}^{\mathrm{td}}}\right|\right)^{\frac{d+2}{3}+\frac{3}{2}}\right)
$$

Proof. Using reductions similar to those in Section 3 , the game on $\widehat{\mathcal{T}}^{\Omega_{\text {rob }}^{\text {td }}}$ can be solved in time $O\left(\left(\left|A_{1}\right|^{*}+\left|A_{2}\right|^{*}\right) \cdot\left(8 \cdot\left|S_{\text {Reg }}^{\widehat{\tau}_{\text {rob }}^{\text {td }}}\right|\right)^{\frac{d+2}{3}+\frac{3}{2}}\right)$ where $S_{\text {Reg }}^{\widehat{\widetilde{T}}_{\text {rob }}^{\text {td }}}$ is the set of regions of $\widehat{\mathcal{T}} \Omega_{\text {rob }}^{\text {td }}$, with $\left|\widehat{\mathcal{T}}_{\text {Reg }}^{\Omega_{\text {rob }}^{\text {td }}}\right|=64 \cdot(|C|+1) \cdot d \cdot\left|S_{\text {Reg }}^{\mathcal{T}}\right| \cdot$ We also need to build a list of regions with valid invariants together with edge constraints satisfied at the region. This takes $O\left(\left|S_{\mathrm{Reg}}^{\Omega_{\mathrm{rob}}^{\mathrm{td}}}\right| \cdot\left|\mathcal{T}_{\text {Constr }}\right|\right)$ time.

We say a timed automaton $\mathcal{T}$ is open if all the guards and invariants in $\mathcal{T}$ are from Constr $^{*}(C)$. Note that even though all the guards and invariants are open, a player might still propose moves to closed regions, e.g., consider an edge between two locations $l_{1}$ and $l_{2}$ with the guard $0<x<2$; a player might propose a move from $\left\langle l_{1}, x=0.2\right\rangle$ to $\left\langle l_{2}, x=1\right\rangle$. The following example shows that player 1 might not have a robust winning strategy in an open timed automaton.

Example 4.5. There exists an open timed automaton game $\mathcal{T}$ such that for a reachability objective $\Phi$, player 1 has a receptive winning strategy for $\Phi$ from a state $s$, but does not have a limit-robust receptive strategy for $\Phi$ from $s$.

Consider the open timed automaton game $\mathcal{T}$ of Figure 6. The invariants of all the locations are true everywhere. The objective of player 1 is to reach $l^{2}$. The set of player-1 actions is $\left\{a_{1}^{0}, a_{1}^{1}\right\}$ and the set of player-2 actions is $\left\{a_{2}^{0}, a_{2}^{1}, a_{2}^{2}\right\}$. The location $l^{3}$ and $l^{4}$ are absorbing locations. Consider the location $l^{1}$. If $x \neq 1$, then player 2 can propose a 0 time duration move to either $l^{4}$ or $l^{5}$, and hence prevent player 1 from reaching $l^{2}$. If $x=1$, then player 1 can propose a 0 time duration move to $l^{2}$, which a receptive strategy of player 2 must eventually allow. Now consider the location $l^{0}$. If $x>1$ then player 2 can propose a 0 time duration move to $l^{3}$. If $x \leq 1$, player 1 wins provided that it moves to the location $l^{1}$ exactly when $x=1$. Thus, no limit-robust winning strategy exists from any location (other than trivially from $l^{2}$ ).

4.2. Winning with Bounded Jitter and Response Time. The limit-robust winning strategies described in subsection 4.1 did not have a lower bound on the jitter: player 1 could propose a move $\left\langle[\alpha, \alpha+\varepsilon], a_{1}\right\rangle$ for arbitrarily small $\alpha$ and $\varepsilon$. In some cases, the controller may be required to work with a known jitter, and also a finite response time. Intuitively, the response time is the minimum delay between a discrete action (of either the controller or the environment) and a discrete action of the controller. The response time models the delay between a location change in a timed game, and when the controller is allowed to 


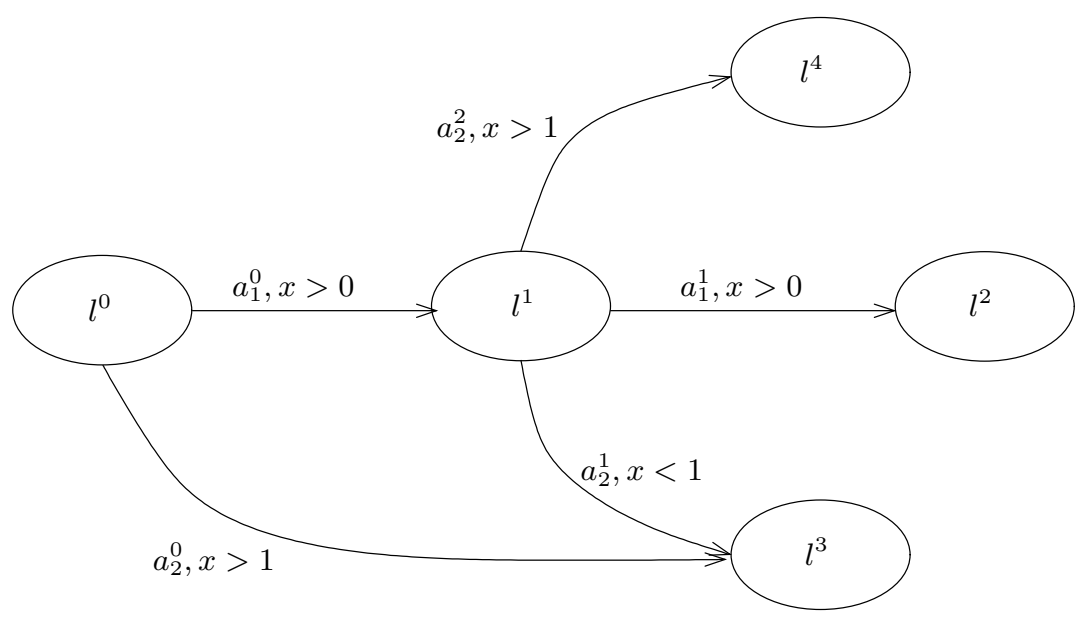

Figure 6: An open timed automaton game $\mathcal{T}$ with no player-1 limit-robust winning strategy.

take an action based on the location change. We incorporate the response time in timed automaton games by restricting player 1 strategies. The jitter is modeled by expanding the set of resulting states to include all the states which lie in a jitter interval around the proposed player-1 delay.

Strategies compatible for $\varepsilon_{\mathrm{j}}$-jitter $\varepsilon_{\mathrm{r}}$-response bounded-robust winning. Given a finite response time $\varepsilon_{\mathrm{r}}$, player 2 can always propose pure time moves of duration $\varepsilon_{\mathrm{r}} / 2$. Thus, if player 1 is restricted to only playing moves of duration longer than $\varepsilon_{\mathrm{r}}$, player 2 can ensure that player-1 moves are never chosen (by repeatedly playing pure time moves, and action moves of duration less than $\varepsilon_{\mathrm{r}}$ ). To allow for such blocking player 2 pure time moves, we only restrict player- 1 strategies to contain moves that are of duration greater than $\varepsilon_{r}$ from the last time a non-pure time move was chosen. In case either player proposes moves such that only time advances, without any discrete action being taken, we adjust the remainder of the response time.

Let $\varepsilon_{\mathrm{j}} \geq 0$ and $\varepsilon_{\mathrm{r}} \geq 0$ be given bounded jitter and response time (we assume both are rational). Formally, a strategy $\pi_{1}$ compatible for $\varepsilon_{\mathrm{j}}$-jitter $\varepsilon_{\mathrm{r}}$-response bounded-robust winning of player 1 proposes a move $\pi_{1}(r[0 . . k])=\left\langle\Delta, a_{1}\right\rangle$ such that if

$$
r[0 . . k]=s_{0},\left\langle m_{1}^{0}, m_{2}^{0}\right\rangle, s_{1},\left\langle m_{1}^{1}, m_{2}^{1}\right\rangle, \ldots, s_{k}
$$

then at least one of the following conditions holds.

- For all $0 \leq j<k$, we have

- if blame ${ }_{i}\left(s_{j}, m_{1}^{j}, m_{2}^{j}, s_{j+1}\right)=$ TRUE, then $m_{i}^{j}=\left\langle\Delta_{i}^{j}, \perp_{i}\right\rangle$ for $i \in\{1,2\}$.

$-\Delta \geq \varepsilon_{\mathbf{r}}-\sum_{j=0}^{k-1} \Delta_{g(j)}^{j}$, where $g(j)= \begin{cases}1 & \text { if blame }{ }_{1}\left(s_{j}, m_{1}^{j}, m_{2}^{j}, s_{j+1}\right)=\text { TRUE } \\ 2 & \text { otherwise. }\end{cases}$

$-\left\{m_{1}^{k}+\epsilon \mid \epsilon \in\left[0, \varepsilon_{\mathrm{j}}\right]\right\} \subseteq \Gamma_{1}\left(s_{k}\right)$.

This corresponds to the case where in the entire run $r[0 . . k]$, (a) no discrete actions have been taken, only simple time moves; (b) player 1 can take a non-pure time move only after $\varepsilon_{r}$ time units from the start of the run; and (c) the moves $m_{1}^{k}+\epsilon$ must be legal player-1 moves for all $\epsilon \in\left[0, \varepsilon_{\mathrm{j}}\right]$.

- There exists a $p$ with $1 \leq p \leq k$ such that all of the following hold. 
- blame $_{i}\left(s_{p-1}, m_{1}^{p-1}, m_{2}^{p-1}, s_{p}\right)=$ TRUE, for $i \in\{1,2\}$, and $m_{i}^{p-1}=\left\langle\Delta_{i}^{p-1}, a_{i}^{p-1}\right\rangle$ with $a_{i}^{p-1} \neq \perp_{i}$ (i.e., the state $r[p]$ arises in the run $r$ due to a non pure-time move).

- For all $p \leq j<k$, if blame ${ }_{i}\left(s_{j}, m_{1}^{j}, m_{2}^{j}, s_{j+1}\right)=$ TRUE then we have $m_{i}^{j}=\left\langle\Delta_{i}^{j}, \perp_{i}\right\rangle$ for $i \in\{1,2\}$ (i.e., only simple time passage moves are taken after the $p-1$-th stage).

$-\Delta \geq \varepsilon_{\mathrm{r}}-\sum_{j=p}^{k-1} \Delta_{g(j)}^{j}$, where $g(j)= \begin{cases}1 & \text { if blame }{ }_{1}\left(s_{j}, m_{1}^{j}, m_{2}^{j}, s_{j+1}\right)=\text { TRUE } \\ 2 & \text { otherwise. }\end{cases}$

$-\left\{m_{1}^{k}+\epsilon \mid \epsilon \in\left[0, \varepsilon_{\mathrm{j}}\right]\right\} \subseteq \Gamma_{1}\left(s_{k}\right)$.

This corresponds to the case where in the run $r[0 . . k]$, (a) the last non-pure move was taken at $r[p-1]$; (b) only simple time passage moves are taken from $r[p-1]$ till $r[k]$; (c) player 1 has to wait $\varepsilon_{\mathrm{r}}$ time units after the discrete action at $r[p-1]$ to propose a new discrete action at $r[k]$; and (c) the moves $m_{1}^{k}+\epsilon$ must be legal player-1 moves for all $\epsilon \in\left[0, \varepsilon_{\mathrm{j}}\right]$.

$\varepsilon_{\mathrm{j}}$-jitter $\varepsilon_{\mathrm{r}}$-response bounded-robust winning. Given a move $m_{1}=\left\langle\Delta, a_{1}\right\rangle$ of player 1 and a move $m_{2}$ of player 2 , the set of $\varepsilon_{\mathrm{j}}$-jittered states is given by $\left\{\delta_{\mathrm{jd}}\left(s, m_{1}+\epsilon, m_{2}\right)\right.$ $\left.\epsilon \in\left[0, \varepsilon_{\mathrm{j}}\right]\right\}$. Given a player-1 strategy $\pi_{1}$ compatible for $\varepsilon_{\mathrm{j}}$-jitter $\varepsilon_{\mathrm{r}}$-response bounded-robust winning of player 1 , and a strategy $\pi_{2}$ of player 2 , the set of possible outcomes in the present semantics is denoted by Outcomes ${ }_{j r}\left(s, \pi_{1}, \pi_{2}\right)$. Each run $r$ in Outcomes $j r\left(s, \pi_{1}, \pi_{2}\right)$ is such that for all $k \geq 0$, the state $r[k+1]$ belongs to the set of $\varepsilon_{\mathrm{j}}$-jittered states arising due to the moves $\pi_{1}(r[0 . . k])$ and $\pi_{2}(r[0 . . k])$ of player 1 and player 2 respectively. We denote the $\varepsilon_{\mathrm{j}}$-jitter $\varepsilon_{\mathrm{r}}$-response bounded-robust winning set for player 1 for an objective $\Phi$ given finite $\varepsilon_{\mathrm{j}}$ and $\varepsilon_{\mathrm{r}}$ by JRWinTimeDiv ${ }_{1}^{\mathcal{T}, \varepsilon_{\mathrm{j}}, \varepsilon_{\mathrm{r}}}(\Phi)$.

The timed automaton $\mathcal{T}^{\varepsilon_{j}, \varepsilon_{r}}$ for computing JRWinTimeDiv ${ }_{1}^{\mathcal{T}, \varepsilon_{\mathrm{j}}, \varepsilon_{\mathrm{r}}}(\Phi)$. We now show that JRWinTimeDiv ${ }_{1}^{\mathcal{T}_{1}, \varepsilon_{\mathrm{j}}, \varepsilon_{\mathrm{r}}}(\Phi)$ can be computed by obtaining a timed automaton $\mathcal{T}^{\mathcal{\varepsilon}_{\mathrm{j}}, \varepsilon_{\mathrm{r}}}$ from $\mathcal{T}$ such that WinTimeDiv ${ }_{1}^{\mathcal{T}_{\mathrm{j}}, \varepsilon_{\mathrm{r}}}(\Phi)=\operatorname{JRWinTimeDiv}_{1}^{\mathcal{T}, \varepsilon_{\mathrm{j}}, \varepsilon_{\mathrm{r}}}(\Phi)$. Given a clock constraint $\varphi$ we make the clocks appearing in $\varphi$ explicit by denoting the constraint as $\varphi(\vec{x})$ for $\vec{x}=$ $\left[x_{1}, \ldots, x_{n}\right]$. Given a real number $\delta$, we let $\varphi(\vec{x}+\delta)$ denote the clock constraint $\varphi^{\prime}$ where $\varphi^{\prime}$ is obtained from $\varphi$ by syntactically substituting $x_{j}+\delta$ for every occurrence of $x_{j}$ in $\varphi$. Let $f^{\varepsilon_{\mathrm{j}}}$ : Constr $(C) \mapsto \operatorname{Constr}(C)$ be a function defined by $f^{\varepsilon_{\mathrm{j}}}(\varphi(\vec{x}))=$ ElimQuant $\left(\forall \delta\left(0 \leq \delta \leq \varepsilon_{\mathrm{j}} \rightarrow \varphi(\vec{x}+\delta)\right)\right)$, where ElimQuant is a function that eliminates quantifiers (this function exists as we are working in the theory of reals with addition, which admits quantifier elimination). The formula $f^{\varepsilon_{\mathrm{j}}}(\varphi)$ ensures that $\varphi$ holds at all the points in $\left\{\vec{x}+\Delta \mid \Delta \leq \varepsilon_{\mathrm{j}}\right\}$.

We now describe the timed automaton $\mathcal{T}^{\varepsilon_{j}, \varepsilon_{r}}$ such that

$$
\operatorname{WinTimeDiv}_{1}^{\mathcal{T}_{\mathrm{j}}, \varepsilon_{\mathrm{r}}}(\Phi)=\mathrm{JRWinTimeDiv}_{1}^{\mathcal{T}, \varepsilon_{\mathrm{j}}, \varepsilon_{\mathrm{r}}}(\Phi)
$$

The automaton has an extra clock $z$ in addition to the clocks of $\mathcal{T}$.

Locations: Corresponding to each location $l$ of $\mathcal{T}$ with outgoing player- 1 edges $e_{1}^{1}, \ldots, e_{1}^{m}$, the automaton $\mathcal{T}^{\varepsilon_{\mathrm{j}}, \varepsilon_{\mathrm{r}}}$ has $m+1$ locations: $l, l_{e_{1}^{1}}, \ldots, l_{e_{1}^{m}}$. The invariant for $l$ is the same as the invariant for $l$ in $\mathcal{T}$. The invariant for $l_{e_{1}^{k}}$ is $z \leq \varepsilon_{\mathrm{j}}$ for all $k$.

Actions: The automaton $\mathcal{T}^{\varepsilon_{j}}, \varepsilon_{\mathrm{r}}$ has the following actions:

- The set of actions for player 1 is $\{\langle 1, e\rangle \mid e$ is a player-1 edge in $\mathcal{T}\}$.

- The set of actions for player 2 is $A_{2}^{\mathcal{T}} \cup\left\{\left\langle a_{2}, e\right\rangle \mid a_{2} \in A_{2}^{\mathcal{T}}\right.$ and $e$ is a player-1 edge in $\left.\mathcal{T}\right\} \cup$ $\{\langle 2, e\rangle \mid e$ is a player-1 edge in $\mathcal{T}\}$ (we assume the unions are disjoint). 
Edges: The automaton $\mathcal{T}^{\varepsilon_{j}, \varepsilon_{\mathrm{r}}}$ has the following edges (every edge includes $z$ in the reset set):

- For $\left\langle l, a_{2}, \varphi, l^{\prime}, \lambda\right\rangle$ a player-2 edge of $\mathcal{T}$, the automaton $\mathcal{T}^{\varepsilon_{\mathrm{j}}, \varepsilon_{\mathrm{r}}}$ contains the player-2 edge $\left\langle l, a_{2}, \varphi, l^{\prime}, \lambda \cup\{z\}\right\rangle$.

- For every player-1 edge $e_{j}=\left\langle l, a_{1}^{j}, \varphi, l^{\prime}, \lambda\right\rangle$ of $\mathcal{T}$, the location $l$ of $\mathcal{T}^{\varepsilon_{\mathrm{j}}, \varepsilon_{\mathrm{r}}}$ has the outgoing player-1 edge $\left\langle l,\left\langle 1, e_{j}\right\rangle, f^{\varepsilon_{\mathrm{j}}}\left(\gamma^{\mathcal{T}}(l)\right) \wedge\left(z \geq \varepsilon_{\mathrm{r}}\right) \wedge f^{\varepsilon_{\mathrm{j}}}(\varphi), l_{e_{j}}, \lambda \cup\{z\}\right\rangle$.

- For $\left\langle l, a_{2}, \varphi_{2}, l^{\prime}, \lambda\right\rangle$ a player-2 edge of $\mathcal{T}$ and $e_{j}$ a player-1 edge from $l$, the location $l_{e_{j}}$ of the automaton $\mathcal{T}^{\varepsilon_{j}, \varepsilon_{r}}$ has a player-2 edge $\left\langle l_{e_{j}},\left\langle a_{2}, e_{j}\right\rangle, \varphi_{2}, l^{\prime}, \lambda \cup\{z\}\right\rangle$.

- For every player-1 edge $e_{j}=\left\langle l, a_{1}^{j}, \varphi_{1}, l^{\prime}, \lambda\right\rangle$ of $\mathcal{T}$, the location $l_{e_{j}}$ of $\mathcal{T}^{\varepsilon_{\mathrm{j}}, \varepsilon_{\mathrm{r}}}$ also has an additional outgoing player-2 edge $\left\langle l_{e_{j}},\left\langle 2, e_{j}\right\rangle, \varphi_{1}, l^{\prime}, \lambda \cup\{z\}\right\rangle$.

The automaton $\mathcal{T}^{\varepsilon_{\mathrm{j}}, \varepsilon_{\mathrm{r}}}$ as described above contains the rational constants $\varepsilon_{\mathrm{r}}$ and $\varepsilon_{\mathrm{j}}$. We can change the timescale by multiplying every constant by the least common multiple of the denominators of $\varepsilon_{\mathrm{r}}$ and $\varepsilon_{\mathrm{j}}$ to get a timed automaton with only integer constants.

The role of the different edges in $\mathcal{T}^{\varepsilon_{\mathrm{j}}, \varepsilon_{\mathrm{r}}}$ is described below.

- A player-2 edge in $\mathcal{T}^{\varepsilon_{\mathrm{j}}, \varepsilon_{\mathrm{r}}}$ labelled with $a_{2}$ such that $a_{2} \in A_{2}^{\mathcal{T}}$ corresponds to the player-2 edge labelled $a_{2}$ in $\mathcal{T}$.

- Player 1 moving from $l$ to $l_{e_{j}}$ with the edge labelled $\left\langle 1, e_{j}\right\rangle$ indicates the desire of player 1 to pick the edge $e_{j}$ from location $l$ in the game $\mathcal{T}$. This is possible in $\mathcal{T}$ iff the following conditions hold.

(a) More that $\varepsilon_{r}$ time has passed since the last discrete action.

(b) The edge $e_{j}$ is enabled for at least $\varepsilon_{\mathrm{j}}$ more time units.

(c) The invariant of $l$ is satisfied for at least $\varepsilon_{\mathrm{j}}$ more time units.

These three requirements are captured by the new guard in $\mathcal{T}^{\varepsilon_{\mathrm{j}}, \varepsilon_{\mathrm{r}}}$, namely $\left(z \geq \varepsilon_{\mathrm{r}}\right) \wedge$ $f^{\varepsilon_{\mathrm{j}}}(\varphi) \wedge f^{\varepsilon_{\mathrm{j}}}\left(\gamma^{\mathcal{T}}(l)\right)$

- Consider a location $l_{e_{j}}$ in $\mathcal{T}^{\varepsilon_{\mathrm{j}}, \varepsilon_{\mathrm{r}}}$. If the game is at $l_{e_{j}}$, then it corresponds to the situation in $\mathcal{T}$ where player 1 has picked the edge labelled $e_{j}$ from location $l$, and it is up to player 2 to allow it or not. The presence of jitter in $\mathcal{T}$ causes uncertainty in when exactly the edge $e_{j}$ is taken. This is modeled in $\mathcal{T}^{\varepsilon_{\mathrm{j}}, \varepsilon_{\mathrm{r}}}$ by having the location $l_{e_{j}}$ be controlled entirely by player 2 for a duration of $\varepsilon_{\mathrm{j}}$ time units. Within $\varepsilon_{\mathrm{j}}$ time units, player 2 must either:

- propose a move $\left\langle a_{2}, e_{j}\right\rangle$ (corresponding to one of its own moves $a_{2}$ in $\mathcal{T}$ ), or, - allow the action $\left\langle 2, e_{j}\right\rangle$ (corresponding to the original player-1 edge $e_{j}$ ) to be taken.

Given a parity function $\Omega^{\mathcal{T}}$ on $\mathcal{T}$, the parity function $\Omega^{\mathcal{T}^{\varepsilon_{j}, \varepsilon_{r}}}$ on $\mathcal{T}^{\varepsilon_{j}, \varepsilon_{r}}$ is given by $\Omega^{\mathcal{T}^{\varepsilon_{j}}, \varepsilon_{r}}(l)=$ $\Omega^{\mathcal{T}^{\mathcal{E}_{j}, \varepsilon_{\mathrm{r}}}}\left(l_{e_{j}}\right)=\Omega^{\mathcal{T}}(l)$. In computing the winning set for player 1 , we need to modify blame ${ }_{1}$ for technical reasons. Whenever an action of the form $\left\langle 1, e_{j}\right\rangle$ is taken, we blame player 2 (even though the action is controlled by player 1$)$; and whenever an action of the form $\left\langle 2, e_{j}\right\rangle$ is taken, we blame player 1 (even though the action is controlled by player 2). Player 2 is blamed as usual for the actions $\left\langle a_{2}, e_{j}\right\rangle$. This modification is needed because player 1 taking the edge $e_{j}$ in $\mathcal{T}$ is broken down into two stages in $\mathcal{T}^{\varepsilon_{\mathrm{j}}, \varepsilon_{\mathrm{r}}}$. If player 1 were to be blamed for the edge $\left\langle 1, e_{j}\right\rangle$, then the following could happen:

(a) player 1 takes the edge $\left\langle 1, e_{j}\right\rangle$ in $\mathcal{T}^{\varepsilon_{j}, \varepsilon_{r}}$ corresponding to its intention to take the edge $e_{j}$ in $\mathcal{T}$, and

(b) player 2 then proposes its own move $\left\langle a_{2}, e_{j}\right\rangle$ from $l_{e_{j}}$, corresponding to it blocking the move $e_{j}$ by $a_{2}$ in $\mathcal{T}$.

If the preceding scenario happens infinitely often, player 1 gets blamed infinitely often even though all it has done is signal its intentions infinitely often, but its actions have not been 


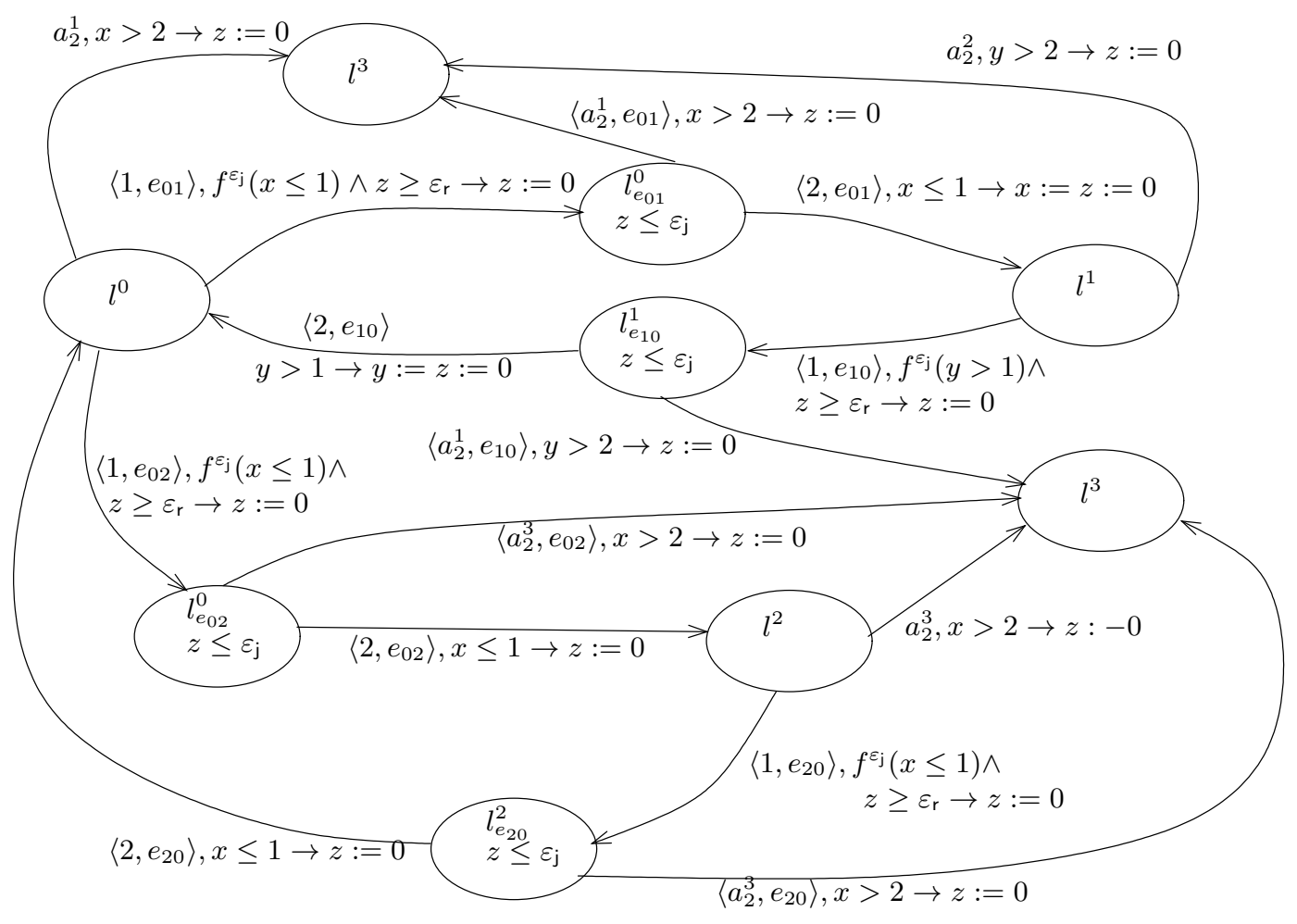

Figure 7: The timed automaton game $\mathcal{T}^{\varepsilon_{j}, \varepsilon_{\mathrm{r}}}$ obtained from $\mathcal{T}$.

chosen. Hence player 2 is blamed for the edge $\left\langle 1, e_{j}\right\rangle$. If player 2 allows the intended player 1 edge by taking $\left\langle 2, e_{j}\right\rangle$, then we must blame player 1 . We note that this modification is not required if $\varepsilon_{\mathrm{r}}>0$, as in this case player-1 signalling its moves infinitely often via moves of the type $\left\langle 1, e_{j}\right\rangle$ can only happen if time progresses by $\varepsilon_{r}$ infinitely often, which implies time divergence. The construction of $\mathcal{T}^{\varepsilon_{j}, \varepsilon_{r}}$ can be simplified if $\varepsilon_{\mathrm{j}}=0$ (then we do not need locations of the form $l_{e_{j}}$ ).

Example 4.6 (Construction of $\mathcal{T}^{\varepsilon_{\mathrm{j}}, \varepsilon_{\mathrm{r}}}$ ). An example of the construction is given in Figure 7 , corresponding to the timed automaton of Figure 1. For the automaton $\mathcal{T}$, we have $A_{1}=$ $\left\{a_{1}^{1}, a_{1}^{2}, a_{1}^{3}, a_{1}^{4}\right\}$ and $A_{2}=\left\{a_{2}^{1}, a_{2}^{2}, a_{2}^{3}\right\}$. The invariants of the locations of $\mathcal{T}$ are all truE. Since $\mathcal{T}$ has at most a single edge from any location $l^{j}$ to $l^{k}$, all edges can be denoted as $e_{j k}$. The set of player- 1 edges is then $\left\{e_{01}, e_{02}, e_{20}, e_{10}\right\}$. The location $l^{3}$ has been replicated for ease of drawing in $\mathcal{T}^{\varepsilon_{\mathrm{j}}, \varepsilon_{\mathrm{r}}}$. The location $l^{3}$ is also an absorbing location - it only has self-loops (we omit these self loops in the figures for simplicity). Observe that $f^{\varepsilon_{\mathrm{j}}}(x \leq 1)=x \leq 1-\varepsilon_{\mathrm{j}}$ and $f^{\varepsilon_{\mathrm{j}}}(y>1)=y>1$.

Given a set of states $\widetilde{S}$ of $\mathcal{T}^{\varepsilon_{\mathrm{j}}, \varepsilon_{\mathrm{r}}}$, let JStates $(\widetilde{S})$ denote the projection of states to $\mathcal{T}$, defined formally by JStates $(\widetilde{S})=\{\langle l, \kappa\rangle \mid l$ is a location of $\mathcal{T}$ and $\langle l, \widetilde{\kappa}\rangle \in \widetilde{S}$ such that $\kappa(x)=$ $\widetilde{\kappa}(x)$ for all $x \in C$, where $C$ is the set of clocks of $\mathcal{T}$. The next theorem states that the timed automaton game $\mathcal{T}^{\varepsilon_{j}, \varepsilon_{\mathrm{r}}}$ can be used to compute the winning states in $\mathcal{T}$ from which player 1 has an $\varepsilon_{\mathrm{j}}$-jitter $\varepsilon_{\mathrm{r}}$-response bounded-robust winning strategy. The proof of correctness of the construction follows from the arguments given in the description of $\mathcal{T}^{\varepsilon_{j}, \varepsilon_{r}}$. 
Theorem 4.7. Let $\mathcal{T}$ be a timed automaton game, $\varepsilon_{\mathrm{r}} \geq 0$ the response time of player 1 , and $\varepsilon_{\mathrm{j}} \geq 0$ the jitter of player 1 actions such that both $\varepsilon_{\mathrm{r}}$ and $\varepsilon_{\mathrm{j}}$ are rational constants. Then, for any $\omega$-regular location objective $\operatorname{Parity}\left(\Omega^{\mathcal{T}}\right)$ of $\mathcal{T}$, we have

$$
\text { JStates }\left(\llbracket z=0 \rrbracket \cap \operatorname{WinTimeDiv}_{1}^{\mathcal{T}^{\mathcal{E}_{\mathrm{j}}, \varepsilon_{\mathrm{r}}}}\left(\operatorname{Parity}\left(\Omega^{\mathcal{T}^{\mathcal{E}_{\mathrm{j}}, \varepsilon_{\mathrm{r}}}}\right)\right)\right)=\operatorname{JRWinTimeDiv}_{1}^{\mathcal{T}_{1}, \varepsilon_{\mathrm{j}}, \varepsilon_{\mathrm{r}}}\left(\operatorname{Parity}\left(\Omega^{\mathcal{T}}\right)\right) \text {, }
$$

where $\mathrm{JRWinTimeDiv}_{1}^{\mathcal{T}, \varepsilon_{\mathrm{j}}, \varepsilon_{\mathrm{r}}}(\Phi)$ is the winning set in the jitter-response semantics, $\mathcal{T}^{\varepsilon_{\mathrm{j}}, \varepsilon_{\mathrm{r}}}$ is the timed automaton with the parity function $\Omega^{\mathcal{T}^{\mathcal{E}_{j}, \varepsilon_{\mathrm{r}}}}$ described above, and $\llbracket z=0 \rrbracket$ is the set of states of $\mathcal{T}^{\varepsilon_{\mathrm{j}}, \varepsilon_{\mathrm{r}}}$ with $\widetilde{\kappa}(z)=0$.

Theorem 4.8. Let $\mathcal{T}$ be a timed automaton game, $\varepsilon_{\mathrm{r}} \geq 0$ the response time of player 1 , and $\varepsilon_{\mathrm{j}} \geq 0$ the jitter of player 1 actions such that both $\varepsilon_{\mathrm{r}}$ and $\varepsilon_{\mathrm{j}}$ are rational constants. Then, for any $\omega$-regular location objective $\operatorname{Parity}\left(\Omega^{\mathcal{T}}\right)$ of $\mathcal{T}$, the winning set

$$
J_{R W i n T i m e D i v}{ }_{1}^{\mathcal{T}, \varepsilon_{\mathrm{j}}, \varepsilon_{\mathrm{r}}}\left(\operatorname{Parity}\left(\Omega^{\mathcal{T}}\right)\right)
$$

can be computed in time

$$
\left.O\left(\left(\left|S_{\text {Reg }}^{\mathcal{T}, \varepsilon_{\mathrm{j}}, \varepsilon_{\mathrm{r}}, \Omega}\right| \cdot\left|\mathcal{T}_{\text {Constr }}\right|^{2}\right)\right)+\left|A_{1}\right| \cdot\left|A_{2}\right| \cdot\left(8 \cdot\left|S_{\mathrm{Reg}}^{\mathcal{T}, \varepsilon_{\mathrm{j}}, \varepsilon_{\mathrm{r}}, \Omega}\right|\right)^{\frac{d+2}{3}+\frac{3}{2}}\right)
$$

where $S_{\mathrm{Reg}}^{\mathcal{T}, \varepsilon_{\mathrm{j}}, \varepsilon_{\mathrm{r}}, \Omega}$ is the set of regions of $\mathcal{T}^{\varepsilon_{\mathrm{j}}, \varepsilon_{\mathrm{r}}}$ with

$$
\begin{aligned}
\left|S_{\mathrm{Reg}}^{\mathcal{T}, \varepsilon_{\mathrm{j}}, \varepsilon_{\mathrm{r}}, \Omega}\right|= & \left|S_{\mathrm{Reg}}^{\mathcal{T}}\right| \cdot 128 \cdot(|C|+1) \cdot(|C|+2) \cdot d \cdot\left(\left|A_{1}\right|+1\right) \cdot \\
& \cdot \max \left(\operatorname{num}\left(\varepsilon_{\mathrm{j}}\right), \operatorname{num}\left(\varepsilon_{\mathrm{r}}\right)\right) \cdot\left(\operatorname{Icm}\left(\operatorname{denom}\left(\varepsilon_{\mathrm{r}}\right), \operatorname{denom}\left(\varepsilon_{\mathrm{j}}\right)\right)\right)^{|C|+1}
\end{aligned}
$$

in which

- $S_{\operatorname{Reg}}^{\mathcal{T}}$ is the set of regions of $\mathcal{T}$;

- $\operatorname{Icm}()$ is the least common multiple function, $\operatorname{denom}()$ and num() are the denominator and numerator functions respectively;

- $\left|\mathcal{T}_{\text {Constr }}\right|$ is the length of the clock constraints in $\mathcal{T}$.

Proof. Let the timed automaton game $\mathcal{T}$ have $|L|$ locations and $\left|A_{i}\right|$ player- $i$ edges for $i \in\{1,2\}$. The automaton $\mathcal{T}^{\varepsilon_{\mathrm{j}}, \varepsilon_{\mathrm{r}}}$ has $|L| \cdot\left(1+\left|A_{1}\right|\right)$ locations, $\left(\left|A_{1}\right|+\left|A_{2}\right|+\left|A_{1}\right| \cdot\left|A_{2}\right|\right)$ player-2 edges, and $\left|A_{1}\right|$ player- 1 edges. Given rational constants $\varepsilon_{\mathrm{j}}$ and $\varepsilon_{\mathrm{r}}$, all the constants in the system need to be multiplied by the least common multiple of the denominators of $\varepsilon_{\mathrm{j}}$ and $\varepsilon_{\mathrm{r}}$. The timed parity game $\mathcal{T}^{\varepsilon_{\mathrm{j}}, \varepsilon_{\mathrm{r}}}$ can hence be solved in time

$$
O\left(\left|A_{1}\right| \cdot\left|A_{2}\right| \cdot\left(8 \cdot S_{\mathrm{Reg}}^{\mathcal{T}, \varepsilon_{\mathrm{j}}, \varepsilon_{\mathrm{r}}, \Omega}\right)^{\frac{d+2}{3}+\frac{3}{2}}\right)
$$

For every player-1 edge $e_{j}=\left\langle l, a_{1}^{j}, \varphi, l^{\prime}, \lambda\right\rangle$ we need to obtain $f^{\varepsilon_{j} \cdot \operatorname{lcm}\left(\left\{\operatorname{denom}\left(\varepsilon_{\mathrm{r}}\right), \operatorname{denom}\left(\varepsilon_{\mathrm{j}}\right)\right\}\right)}(\varphi)$. This takes time $O\left(|\varphi|^{2}\right)$ (see $\left[\right.$ BPR03]). We observe that $f^{\varepsilon_{\mathrm{j}} \cdot \operatorname{lcm}\left(\left\{\operatorname{denom}\left(\varepsilon_{\mathrm{r}}\right) \text {,denom }\left(\varepsilon_{\mathrm{j}}\right)\right\}\right)}(\varphi)$ cannot have any constants other than those in $\varphi$, and $\varepsilon_{\mathrm{r}}, \varepsilon_{\mathrm{r}}$ (this can be seen by putting $\varphi$ in a disjunctive normal form and applying a Fourier-Motzkin like quantifier elimination procedure [Sch86]). Thus, building a list of regions of $\mathcal{T}^{\varepsilon_{j}, \varepsilon_{\mathrm{r}}}$ with valid invariants together with edge constraints satisfied at the regions takes time $O\left(\left|S_{\text {Reg }}^{\mathcal{T}, \varepsilon_{\mathrm{j}}, \varepsilon_{\mathrm{r}}, \Omega}\right| \cdot\left|\mathcal{T}_{\text {Constr }}\right|^{2}\right)$ 


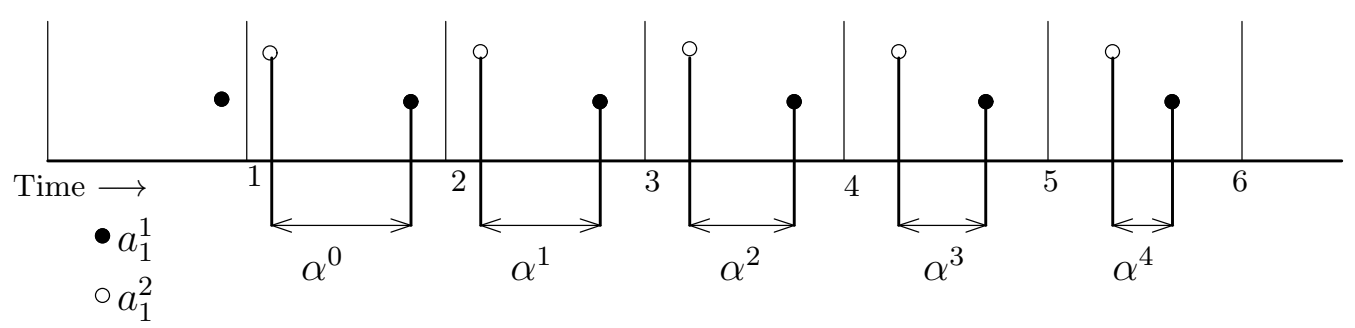

Figure 8: General timeline of a run in the game of Fig. 1 (decreasing sequence of timegaps $\left.\alpha^{j}\right)$.

Example 4.9 (Differences between various winning modes). Consider the timed automaton $\mathcal{T}$ in Fig. 1. Let the objective of player 1 be $\square\left(\neg l^{3}\right)$, i.e., to avoid $l^{3}$. The relevant part of the automaton for this example is the cycle $l^{0}, l^{1}$. The only way to avoid $l^{3}$ in a time divergent run is to cycle in between $l^{0}$ and $l^{1}$ infinitely often. In additional player 1 may choose to also cycle in between $l^{0}$ and $l^{2}$, but that does not help (or harm) it. In our analysis, we omit such $l^{0}, l^{2}$ cycles, noting that our receptive formulation correctly allows any number of $l^{0}, l^{2}$ cycles. We present an intuitive explanation here, a detailed analysis can be found in the appendix.

Let the game start from the location $l^{0}$. In a run $r$, let $t_{1}^{j}$ and $t_{2}^{j}$ be the times when the $a_{1}^{1}$ transition and the $a_{1}^{2}$ transitions respectively are taken for the $j$-th time. The timeline is depicted in Figure 8. The guards $x \leq 1$ on $a_{1}^{1}$ and $y>1$ on $a_{1}^{2}$ ensure that the distance between the $j+1$-th $a_{1}^{1}$ transition, and the $j$-th $a_{1}^{2}$ transition keeps on strictly decreasing with increasing $j$. To see this, observe that $t_{1}^{j+1}-t_{1}^{j} \leq 1$ because of the guard $x \leq 1$, and $t_{2}^{j}-t_{2}^{j-1}>1$ because of the guard $y>1$. Rearranging, we get $t_{1}^{j+1}-t_{2}^{j}<t_{1}^{j}-t_{2}^{j-1}$. Consider the time gap sequence $\alpha^{j+1}=t_{1}^{j+1}-t_{2}^{j}$, i.e., the sequence of time gaps between the $j+1$-th $a_{1}^{1}$ transition, and the $j$-th $a_{1}^{2}$ transition. For any $\epsilon$-jitter strategy of player 1 with $\epsilon>0$, this sequence must decrease by more than $\epsilon$ for each step, which clearly cannot happen infinitely often since $\alpha^{j}$ must be positive for all $j$, as the $j+1$-th $a_{1}^{1}$ transition must always happen after the $j$-th $a_{1}^{2}$ transition. Thus, player 1 has no $\epsilon$-jitter bounded-robust winning strategy from $l^{0}$. Player 1 does however have a limit-robust strategy, as a limit-robust strategy can be such that the time gap sequence $\alpha^{j}$ decreases, but by a smaller and smaller amount at each step, ensuring that $\alpha^{j}$ stays positive for all $j$. This shows that $\epsilon$-jitter bounded-robust winning strategies for $\epsilon>0$ are strictly less powerful than limit-robust strategies.

To see that limit-robust strategies are strictly less powerful than general receptive strategies, observe that player 1 does not have a winning limit-robust strategy from $\left\langle l^{0}, x=y=1\right\rangle$ as it would have to take the first $a_{1}^{1}$ transition immediately. It can be shown that there exists a winning player- 1 receptive strategy from $\left\langle l^{0}, x=y=1\right\rangle$.

The following theorem follows from the fact that bounded-robust winning strategies are no more powerful than limit-robust strategies, which are in turn no more powerful than general receptive strategies. The strictness of the inclusions can be observed in Example 4.9.

Theorem 4.10. Let $\mathcal{T}$ be a timed automaton and $\Phi$ an objective. For all $\varepsilon_{\mathrm{j}}>0$ and $\varepsilon_{\mathrm{r}} \geq 0$, we have JRWinTimeDivivi, $\varepsilon_{1}^{\varepsilon_{j}, \varepsilon_{r}}(\Phi) \subseteq \operatorname{RobWinTimeDiv}_{1}(\Phi) \subseteq \operatorname{WinTimeDiv}_{1}(\Phi)$. All the subset inclusions are strict in general. 
Sampling semantics. Instead of having a response time for actions of player 1 , we can have a model where player 1 is only able to take actions in an $\varepsilon_{\mathrm{j}}$ interval around sampling times, with a given time period $\varepsilon_{\text {sample }}$. A timed automaton can be constructed along similar lines to that of $\mathcal{T}^{\varepsilon_{j}, \varepsilon_{r}}$ to obtain the winning set.

\section{REFERENCES}

[AD94] R. Alur and D.L. Dill. A theory of timed automata. Theor. Comput. Sci., 126(2):183-235, 1994.

[AdAF05] B. Adler, L. de Alfaro, and M. Faella. Average reward timed games. In FORMATS 05, LNCS 3829, pages 65-80. Springer, 2005.

[AH97] R. Alur and T.A. Henzinger. Modularity for timed and hybrid systems. In CONCUR 97, LNCS 1243, pages 74-88. Springer, 1997.

[AT04] M. Agrawal and P.S. Thiagarajan. Lazy rectangular hybrid automata. In HSCC, LNCS 2993, pages $1-15,2004$.

[ATM05] R. Alur, S.L. Torre, and P. Madhusudan. Perturbed timed automata. In HSCC, LNCS 3414, pages 70-85. Springer, 2005.

[BMR08] P. Bouyer, N. Markey, and P.A. Reynier. Robust analysis of timed automata via channel machines. In FoSSaCS 08, LNCS 4962, pages 157-171. Springer, 2008.

[BPR03] S. Basu, R. Pollack, and M.F. Roy. Algorithms in Real Algebraic Geometry. Springer, 2003.

$\left[\mathrm{CDF}^{+} 05\right] \quad$ F. Cassez, A. David, E. Fleury, K.G. Larsen, and D. Lime. Efficient on-the-fly algorithms for the analysis of timed games. In CONCUR 05, LNCS 3653, pages 66-80. Springer, 2005.

[CHP08] K. Chatterjee, T.A. Henzinger, and V.S. Prabhu. Timed parity games: Complexity and robustness. In FORMATS 08, LNCS 5215, pages 124-140. Springer, 2008.

$\left[\mathrm{dAFH}^{+} 03\right]$ L. de Alfaro, M. Faella, T.A. Henzinger, R. Majumdar, and M. Stoelinga. The element of surprise in timed games. In CONCUR 03, LNCS 2761, pages 144-158. Springer, 2003.

[dAHM01] L. de Alfaro, T.A. Henzinger, and R. Majumdar. From verification to control: Dynamic programs for omega-regular objectives. In LICS 01, pages 279-290. IEEE Computer Society Press, 2001.

[DM02] D. D'Souza and P. Madhusudan. Timed control synthesis for external specifications. In STACS 02, LNCS 2285, pages 571-582. Springer, 2002.

[FTM02a] M. Faella, S. La Torre, and A. Murano. Automata-theoretic decision of timed games. In VMCAI 02, LNCS 2294, pages 94-108. Springer, 2002.

[FTM02b] M. Faella, S. La Torre, and A. Murano. Dense real-time games. In LICS 02, pages 167-176. IEEE Computer Society, 2002.

[GHJ97] V. Gupta, T.A. Henzinger, and R. Jagadeesan. Robust timed automata. In HART: Hybrid and Real-Time Systems, LNCS 1201, pages 331-345. Springer, 1997.

[HP06] T.A. Henzinger and V.S. Prabhu. Timed alternating-time temporal logic. In FORMATS 06, LNCS 4202, pages 1-17. Springer, 2006.

[HR00] T.A. Henzinger and J.-F. Raskin. Robust undecidability of timed and hybrid systems. In $H S C C$, LNCS 1790, pages 145-159. Springer, 2000.

[JKH02] M. Jurdzinski, O. Kupferman, and T. A. Henzinger. Trading probability for fairness. In CSL, LNCS 2471, pages 292-305. Springer, 2002.

[JT07] M. Jurdzinski and A. Trivedi. Reachability-time games on timed automata. In ICALP, LNCS 4596, pages 838-849. Springer, 2007.

[Jur00] M. Jurdzinski. Small progress measures for solving parity games. In STACS, LNCS 1770, pages 290-301. Springer, 2000.

[MPS95] O. Maler, A. Pnueli, and J. Sifakis. On the synthesis of discrete controllers for timed systems (an extended abstract). In STACS 95, pages 229-242, 1995.

[PAMS98] A. Pnueli, E. Asarin, O. Maler, and J. Sifakis. Controller synthesis for timed automata. In Proc. System Structure and Control. Elsevier, 1998.

[Sch86] A. Schrijver. Theory of linear and integer programming. John Wiley \& Sons, Inc., New York, NY, USA, 1986.

[Sch07] S. Schewe. Solving parity games in big steps. In Proc. FST TCS. Springer-Verlag, 2007. 
[SGSAL98] R. Segala, R. Gawlick, J.F. Søgaard-Andersen, and N.A. Lynch. Liveness in timed and untimed systems. Inf. Comput., 141(2):119-171, 1998.

[Tho97] W. Thomas. Languages, automata, and logic. In Handbook of Formal Languages, volume 3, Beyond Words, chapter 7, pages 389-455. Springer, 1997.

[Tri09] A. Trivedi. Competitive Optimisation on Timed Automata. PhD thesis, Department of Computer Science, University of Warwick, April 2009.

[VJ00] J. Vöge and M. Jurdziński. A discrete strategy improvement algorithm for solving parity games. In $C A V^{\prime} 00$, LNCS 1855, pages 202-215. Springer, 2000.

[WDMR04] M. D. Wulf, L. Doyen, N. Markey, and J.F. Raskin. Robustness and implementability of timed automata. In FORMATS/FTRTFT, LNCS 3253, pages 118-133. Springer, 2004.

[WDR05] M. D. Wulf, L. Doyen, and J. F. Raskin. Almost asap semantics: from timed models to timed implementations. Formal Asp. Comput., 17(3):319-341, 2005.

[WH91] H. Wong-Toi and G. Hoffmann. The control of dense real-time discrete event systems. In Proc. of 30th Conf. Decision and Control, pages 1527-1528, 1991. 


\section{Appendix}

\subsection{Proofs of Section 2, Proof of Lemma 2.5.}

Proof. Consider a run $\widehat{r}$ of $\widehat{\mathcal{T}}^{\Omega^{\mathrm{td}}}$. We show that the maximum index visited infinitely often is even iff the run $\widehat{r}$ satisfies $\left((\square \diamond\right.$ tick $\rightarrow$ Parity $(\Omega)) \wedge\left(\neg \square \diamond\right.$ tick $\left.\left.\rightarrow \diamond \square \neg b l_{1}\right)\right)$

$\Rightarrow$ Suppose the run $\widehat{r}$ satisfies $\left((\square \diamond\right.$ tick $\rightarrow$ Parity $(\Omega)) \wedge\left(\neg \square \diamond\right.$ tick $\left.\left.\rightarrow \diamond \square \neg b l_{1}\right)\right)$ We show the maximum index visited infinitely often is even. The following cases can arise.

(a) The run $\widehat{r}$ satisfies $\neg \square \diamond$ tick and $\diamond \square \neg b l_{1}$, i.e., $\widehat{r}[j]$ has tick $=b l_{1}=$ false for all $j \geq n$ for some $n$. In this case the parity seen infinitely often is 0 (even).

(b) The run $\widehat{r}$ satisfies $\square \diamond$ tick and also belongs to Parity $(\Omega)$. The component $p$ of the state in $\widehat{\mathcal{T}}^{\Omega^{\text {td }}}$ remembers the maximum $\Omega$ index seen since the state following the last occurrence of tick = TRUE. That is, in a run $\widehat{r}$, if $\widehat{r}[j]$ has tick = TRUE, and $\widehat{r}[j+1], \ldots \widehat{r}[j+m]$ all have tick = FALSE (except possibly for $\widehat{r}[j+m]$ ), then for $j+1 \leq k \leq j+m$ the value of $p$ in $\widehat{r}[k]$ is equal to $\max \{\Omega(\widehat{r}[i]) \mid j+1 \leq i \leq k\}$. Since tick is true infinitely often, the maximum $\Omega^{\text {td }}$ index seen infinitely often is $m+2$ where $m$ is the maximum $\Omega$ index seen infinitely often in $\widehat{r}$. Since $\widehat{r}$ belongs to $\operatorname{Parity}(\Omega), m$ is even. Thus the parity seen infinitely often is $m+2$ (even).

$\Leftarrow$ Suppose the run $\widehat{r}$ does not satisfy $\left((\square \diamond\right.$ tick $\rightarrow$ Parity $(\Omega)) \wedge\left(\neg \square \diamond\right.$ tick $\left.\left.\rightarrow \diamond \square \neg b l_{1}\right)\right)$.

We show the maximum index visited infinitely often is odd. The following cases can arise.

(a) The run $\widehat{r}$ satisfies $\neg \square \diamond$ tick and $\square \diamond b l_{1}$. In this case tick $=$ false for all $j \geq n$ for some $n$; and $b l_{1}=$ TRUE infinitely often. Thus, the maximum index seen infinitely often is 1 .

(b) The run $\widehat{r}$ satisfies $\square \diamond$ tick and also belongs to Parity $(\Omega)$. As above, the maximum $\Omega^{\text {td }}$ index seen infinitely often in $\widehat{r}$ is $m+2$ where $m$ is the maximum $\Omega$ index seen infinitely often in $\widehat{r}$. Since $\widehat{r}$ does not belong to Parity $(\Omega), m$ is odd. Thus the parity seen infinitely often is $m+2$ (odd).

\section{Proof of Lemma 2.7.}

Proof. Consider the $\mu$-calculus formula $\varphi$ for describing the winning set $\operatorname{Win}_{1}^{\widehat{\widetilde{T}}^{\Omega^{\mathrm{td}}}}\left(\operatorname{Parity}\left(\Omega^{\mathrm{td}}\right)\right)$. The formula contains the CPre ${ }_{1}$ operator. The set $\mathrm{CPre}_{1}(Z)$ remains unchanged (for $Z \mathrm{a}$ union of regions of $\widehat{\mathcal{T}}^{\Omega^{\mathrm{td}}}$ ) if player 1 is restricted to use only memoryless strategies. Suppose $\widehat{s} \in \mathrm{CPre}_{1}(Z)$, and let $m_{1}^{\widehat{s}}$ be the winning move of player 1 from $\widehat{s}$ such that no matter what player 2 does, the next state lies in $Z$. Let $\widehat{R}_{1}=\operatorname{Reg}\left(\delta\left(\widehat{s}, m_{1}^{\widehat{s}}\right)\right)$, and let the available moves of player 2 from $\widehat{s}$ be to regions $\widehat{R}_{2}^{1}, \ldots, \widehat{R}_{2}^{n}$. We have that from any state in $\operatorname{Reg}(\widehat{s})$, player 1 has a move to $\widehat{R}_{1}$, and that player 2 can only take moves to $\widehat{R}_{2}^{1}, \ldots, \widehat{R}_{2}^{n}$. From Lemma 3.1 , it then follows that if player 1 proposes a move to $\widehat{R}_{1}$ from any state in $\operatorname{Reg}(\widehat{s})$, then no matter what player 2 does, the resulting state will lie in $Z$. Thus, memoryless region strategies suffice as winning strategies. Moreover, again from Lemma 3.1, any move of player 1 to the region $\widehat{R}_{1}$ is a winning move. Thus, we have that there is a memoryless region winning strategy $\pi_{1}$ from winning states, and that any strategy region equivalent to $\pi_{1}$ is also a winning strategy. 


\section{Proof of Proposition 2.8,}

Proof. Intuitively, in the structure $\mathcal{T}$, we want player 1 to be able to infer the values corresponding to $\mathfrak{z}, t i c k, b l_{1}, p$. If player 1 can do this, then it can maintain the structure $\widehat{\mathcal{T}}^{\Omega^{\text {td }}}$ in memory, and thus it can use a winning memoryless strategy of $\widehat{\mathcal{T}}^{\Omega^{\text {td }}}$ (by Lemma 2.7 memoryless strategies suffice in $\widehat{\mathcal{T}}^{\Omega^{\mathrm{td}}}$ ). This strategy will then be move-independent. The values of $\mathfrak{z}$ and tick can be inferred from the value of the global clock $z$. And given a state $r[k]$ in a run $r$, and a move $\left\langle\Delta_{1}, a_{1}\right\rangle$ of player 1 , the $b l_{1}$ component will be true iff $(\operatorname{time}(r[k+1])-\operatorname{time}(r[k]))=\Delta_{1}$ and $\delta\left(r[k],\left\langle\Delta_{1}, a_{1}\right\rangle\right)=r[k+1]$. The value of the component $p$ can be inferred from the parity values of $\Omega$, and the values of $t i c k, b l_{1}$.

\section{Proof of Lemma 2.9.}

Proof. Consider the $\mathrm{CPre}_{1}$ operator in the $\mu$-calculus formula for describing the winning set $\operatorname{Win}_{1}^{\widehat{\widetilde{T}}^{\Omega^{\text {td }}}}\left(\operatorname{Parity}\left(\Omega^{\mathrm{td}}\right)\right)$. The set $\mathrm{CPre}_{1}(Z)$ remains unchanged (for $Z$ a union of regions of $\left.\widehat{\mathcal{T}}^{\Omega^{\mathrm{td}}}\right)$ if player 2 is restricted to playing only move-independent region strategies. This is because from it can be shown from Lemma 3.1 that from any state $\widehat{s}$, the ability of player 2 to prevent player 1 from reaching $Z$ in the next step depends only on $\operatorname{Reg}(\widehat{s})$, the regions in $Z$, and the current move of player 1 . Moreover, if player 2 can prevent player 1 from reaching $Z$ from $\widehat{s}$ for all player 1 moves, then there is a unique region $\widehat{R}^{*}$ such that for all $\widehat{s}^{\prime} \in \operatorname{Reg}(\widehat{s})$, against any player 1 move $m_{1}^{\widehat{s}^{\prime}}$, player 2 has a counter-move $m_{2}^{\widehat{s}^{\prime}, m_{1}^{\widehat{s}^{\prime}}}$ with $\delta^{\widehat{\Im}^{\Omega^{\text {td }}}}\left(\widehat{s}^{\prime}, m_{2}^{\widehat{s}^{\prime}, m_{1}^{\widehat{s}^{\prime}}}\right) \in \widehat{R}^{*}$ such that the move $m_{2}^{\widehat{s}^{\prime}, m_{1}^{\widehat{s}^{\prime}}}$ prevents the player-1 move $m_{1}^{\widehat{s}^{\prime}}$ from reaching $Z$. Thus, move-independent region strategies of player 2 suffice as spoiling strategies.

5.2. Proofs of Section 3. We start with the statement of a classical result of AD94 that the region equivalence relation induces a time abstract bisimulation on the regions.

Lemma 5.1 ([AD94] $)$. Let $Y, Y^{\prime}$ be regions in the timed game structure $\mathcal{T}$. Suppose player $i$ has a move from $s_{1} \in Y$ to $s_{1}^{\prime} \in Y^{\prime}$, for $i \in\{1,2\}$. Then, for any $s_{2} \in Y$, player $i$ has a move from $s_{2}$ to some $s_{2}^{\prime} \in Y^{\prime}$.

\section{Proof of Lemma 3.1 .}

Proof. From Lemma 5.1, if player $i$ has a move from some $s_{1} \in Y$ to $s_{1}^{\prime} \in Y^{\prime}$, for $i \in\{1,2\}$. Then, for any $s_{2} \in Y$, player $i$ has a move from $s_{2}$ to some $s_{2}^{\prime} \in Y^{\prime}$.

Consider the case when $Y_{1}^{\prime} \neq Y_{2}^{\prime}$. The proof follows from the fact that each region has a unique first time-successor region. Thus, if $Y_{1}^{\prime}$ is "closer" to $Y$ than $Y_{2}^{\prime}$, then the move of player 1 wins, otherwise, the move of player 2 wins. A region $R^{\prime}$ is a first time-successor of $R \neq R^{\prime}$ if for all states $s \in R$, there exists $\Delta>0$ such that $s+\Delta \in R^{\prime}$ and for all $\Delta^{\prime}<\Delta$, we have $s+\Delta^{\prime} \in R \cup R^{\prime}$. The time-successor of $\langle l, h, \mathcal{P}(C)\rangle$ is $\left\langle l, h^{\prime}, \mathcal{P}^{\prime}(C)\right\rangle$ when

- $h=h^{\prime}, \mathcal{P}(C)=\left\langle C_{-1}, C_{0} \neq \emptyset, C_{1}, \ldots, C_{n}\right\rangle$, and $\mathcal{P}^{\prime}(C)=\left\langle C_{-1}, C_{0}^{\prime}=\emptyset, C_{1}^{\prime}, \ldots, C_{n+1}^{\prime}\right\rangle$ where $C_{i}^{\prime}=C_{i-1}$, and $h(x)<c_{x}$ for every $x \in C_{0}$.

- $h=h^{\prime}, \mathcal{P}(C)=\left\langle C_{-1}, C_{0} \neq \emptyset, C_{1}, \ldots, C_{n}\right\rangle$, and $\mathcal{P}^{\prime}(C)=\left\langle C_{-1}^{\prime}=C_{-1} \cup C_{0}, C_{0}^{\prime}=\right.$ $\left.\emptyset, C_{1}, \ldots, C_{n}\right\rangle$, and $h(x) \geq c_{x}$ for every $x \in C_{0}$. 
- $h=h^{\prime}, \mathcal{P}(C)=\left\langle C_{-1}, C_{0} \neq \emptyset, C_{1}, \ldots, C_{n}\right\rangle$, and $\mathcal{P}^{\prime}(C)=\left\langle C_{-1}^{\prime}, C_{0}^{\prime}=\emptyset, C_{1}^{\prime}, \ldots, C_{n+1}^{\prime}\right\rangle$ where $C_{i}^{\prime}=C_{i-1}$ for $i \geq 2, h(x)<c_{x}$ for every $x \in C_{1}^{\prime} \subseteq C_{0}$, and $h(x) \geq c_{x}$ for every $x \in C_{0} \backslash C_{1}^{\prime}$, and $C_{-1}^{\prime}=C_{-1} \cup C_{0} \backslash C_{1}^{\prime}$.

- $\mathcal{P}(C)=\left\langle C_{-1}, C_{0}=\emptyset, C_{1}, \ldots, C_{n}\right\rangle, \mathcal{P}^{\prime}(C)=\left\langle C_{-1}, C_{0}^{\prime}=C_{n}, C_{1}, \ldots, C_{n-1}\right\rangle$, and $h^{\prime}(x)=$ $h(x)+1 \leq c_{x}$ for every $x \in C_{n}$, and $h^{\prime}(x)=h(x)$ otherwise.

- $\mathcal{P}(C)=\left\langle C_{-1}, C_{0}=\emptyset, C_{1}, \ldots, C_{n}\right\rangle, \mathcal{P}^{\prime}(C)=\left\langle C_{-1}^{\prime}=C_{-1} \cup C_{n}, C_{0}, C_{1}, \ldots, C_{n-1}\right\rangle$, and $h^{\prime}(x)=h(x)=c_{x}$ for every $x \in C_{n}$, and $h^{\prime}(x)=h(x)$ otherwise.

- $\mathcal{P}(C)=\left\langle C_{-1}, C_{0}=\emptyset, C_{1}, \ldots, C_{n}\right\rangle, \mathcal{P}^{\prime}(C)=\left\langle C_{-1}^{\prime}=C_{-1} \cup C_{n} \backslash C_{0}^{\prime}, C_{0}^{\prime}, C_{1}, \ldots, C_{n-1}\right\rangle$, and $h^{\prime}(x)=h(x)+1 \leq c_{x}$ for every $x \in C_{1}^{\prime} \subseteq C_{n}, h^{\prime}(x)=h(x)=c_{x}$ for every $x \in C_{n} \backslash C_{1}^{\prime}$, and $h^{\prime}(x)=h(x)$ otherwise.

In case $Y_{1}^{\prime}=Y_{2}^{\prime}$, then player 2 can pick the same time to elapse as player 1 , and ensure that the conditions of the lemma hold.

\section{Completion of proof of Lemma 3.5.}

Proof. (Continued).

We constructed the 3 -region strategy $\pi_{1}^{*}$ from $\pi_{1}$, and we claimed $\pi_{1}^{*}$ was a winning strategy. We were proving it by contradiction. We assumed a spoiling strategy $\pi_{2}^{*}$ for $\pi_{1}^{*}$, and we constructed a player-2 strategy $\pi_{2}$ that we claimed was spoiling for $\pi_{1}$. We were showing by induction that there exists a run $\widehat{r}_{3} \in \operatorname{Outcomes}\left(\widehat{s}, \pi_{1}, \pi_{2}\right)$ such that $\widehat{r}_{3}[k]=\widehat{r}_{3}^{*}[k]$ for all $k \geq 0$ ( $\widehat{r}_{3}^{*}$ was the run used in defining $\pi_{2}$, and is such that $\widehat{r}_{3}^{*} \in \operatorname{Outcomes}\left(\widehat{s}, \pi_{1}^{*}, \pi_{2}^{*}\right)$ and $\left.\widehat{r}_{3}^{*} \notin \operatorname{TimeDiv} 3 \mathrm{Bl}_{1}(\operatorname{Parity}(\Omega))\right)$. We present the details of the induction proof. The proof of the above claim is by induction on $k$. For $k=0$ the claim is trivially true. Suppose the claim is true for all $j \leq k$. Thus, we have a run $\widehat{r}_{3}$ such that $\widehat{r}_{3}[j]=\widehat{r}_{3}^{*}[j]$ for all $j \leq k$. We show that the run $\widehat{r}_{3}[0 . . k]$ can be extended to $\widehat{r}_{3}[0 . . k+1]$ according to $\pi_{1}, \pi_{2}$ such that $\widehat{r}_{3}[k+1]=\widehat{r}_{3}^{*}[k+1]$. We have the following cases:

(1) The following hold.

- $\pi_{1}\left(\widehat{r}_{3}[0 . . k]\right)=\left\langle\Delta, a_{1}\right\rangle$, and

- $a_{1}=\perp_{*}$.

Since $\pi_{1}$ is a memoryless strategy of $\widehat{T}_{3 b l}^{\Omega^{\text {td }}}$, we have $\pi_{1}\left(\widehat{r}_{3}^{*}[0 . . k]\right)=\left\langle\Delta, \perp_{*}\right\rangle$. Suppose $\pi_{2}^{*}\left(\widehat{r}_{3}^{*}[0 . . k]\right)$ has the form $\left\langle\Delta_{2}, a_{2}\right\rangle$. By definition of $\pi_{2}$, we have $\pi_{2}\left(\widehat{r}_{3}[0 . . k]\right)=$ $\pi_{2}^{*}\left(\widehat{r}_{3}[0 . . k]\right)=\pi_{2}^{*}\left(\widehat{r}_{3}^{*}[0 . . k]\right)=\left\langle\Delta_{2}, a_{2}\right\rangle$ ( $\pi_{2}^{*}$ is a memoryless strategy). Hence, we have $\widehat{r}_{3}^{*}[k+1]=\delta^{\widehat{\Im}_{3 b l}^{\text {td }}}\left(\widehat{r}_{3}^{*}[k],\left\langle\Delta_{2}, a_{2}\right\rangle\right)=\delta^{\widehat{\Im}_{3 b l}^{\text {td }}}\left(\widehat{r}_{3}[k],\left\langle\Delta_{2}, a_{2}\right\rangle\right)=\widehat{r}_{3}[k+1]$.

(2) The following hold.

- $\pi_{1}\left(\widehat{r}_{3}[0 . . k]\right)=\left\langle\Delta, a_{1}\right\rangle$,

- $a_{1} \neq \perp_{*}$, and

- Succr $\widehat{\widetilde{T}}^{\Omega^{\mathrm{td}}}\left(\widehat{r}_{3}[k], \Delta\right)=$ TRUE

Since $\pi_{1}$ is a memoryless strategy, we have $\pi_{1}\left(\widehat{r}_{3}^{*}[0 . . k]\right)=\left\langle\Delta, a_{1}\right\rangle$. Combining this with Succr $\widehat{\widetilde{T}}_{3}^{\Omega^{\text {td }}}\left(\widehat{r}_{3}^{*}[k], \Delta\right)=$ TRUE, yields $\pi_{1}^{*}\left(\widehat{r}_{3}^{*}[0 . . k]\right)=\pi_{1}\left(\widehat{r}_{3}^{*}[0 . . k]\right)=\left\langle\Delta, a_{1}\right\rangle$. Let $\pi_{2}^{*}\left(\widehat{r}_{3}^{*}[0 . . k]\right)=\left\langle\Delta_{2}, a_{2}\right\rangle$. By definition of $\pi_{2}$, we have $\pi_{2}\left(\widehat{r}_{3}[0 . . k]\right)=\pi_{2}^{*}\left(\widehat{r}_{3}[0 . . k]\right)=$ 


$$
\begin{aligned}
& \pi_{2}^{*}\left(\widehat{r}_{3}^{*}[0 . . k]\right)=\left\langle\Delta_{2}, a_{2}\right\rangle\left(\pi_{2}^{*} \text { is a memoryless strategy }\right) . \text { Thus, } \\
& \delta_{\mathrm{jd}}^{\widehat{\tau}_{3 b l}^{\Omega^{\mathrm{td}}}}\left(\widehat{r}_{3}[k], \pi_{1}\left(\widehat{r}_{3}[0 . . k]\right), \pi_{2}\left(\widehat{r}_{3}[0 . . k]\right)\right)=\delta_{\mathrm{jd}}^{\widehat{\tau}_{3 b l}^{\Omega^{\mathrm{td}}}}\left(\widehat{r}_{3}[k],\left\langle\Delta, a_{1}\right\rangle,\left\langle\Delta_{2}, a_{2}\right\rangle\right) \\
& =\delta_{\mathrm{jd}}^{\widehat{\mathcal{T}}_{3 b l}^{\Omega^{\mathrm{td}}}}\left(\widehat{r}_{3}^{*}[k],\left\langle\Delta, a_{1}\right\rangle,\left\langle\Delta_{2}, a_{2}\right\rangle\right) \\
& =\delta_{\mathrm{jd}}^{\widehat{\mathfrak{T}}_{3 b l}^{\Omega^{\mathrm{td}}}}\left(\widehat{r}_{3}^{*}[k], \pi_{1}^{*}\left(\widehat{r}_{3}^{*}[0 . . k]\right), \pi_{2}^{*}\left(\widehat{r}_{3}^{*}[0 . . k]\right)\right) .
\end{aligned}
$$

Hence $\widehat{r}_{3}[0 . . k]$ can be extended to $\widehat{r}_{3}[0 . . k+1]$ according to $\pi_{1}, \pi_{2}$ such that $\widehat{r}_{3}[0 . . k+1]=$ $\widehat{r}_{3}^{*}[0 . . k+1]$.

(3) The following hold.

- $\pi_{1}\left(\widehat{r}_{3}[0 . . k]\right)=\left\langle\Delta, a_{1}\right\rangle, a_{1} \neq \perp_{*}$,

- $\operatorname{Succr}_{3} \widehat{\widetilde{T}}^{\Omega^{\mathrm{td}}}\left(\widehat{r}_{3}[k], \Delta\right)=$ FALSE,

- $\pi_{1}^{*}\left(\widehat{r}_{3}[0 . . k]\right)=\left\langle\Delta^{\prime}, \perp_{1}\right\rangle$, and

- $\pi_{2}^{*}\left(\widehat{r}_{3}[0 . . k]\right)=\left\langle\Delta^{*}, a_{2}^{*}\right\rangle$ with $\Delta^{*}<\Delta^{\prime}$.

We have $\pi_{2}\left(\widehat{r}_{3}[0 . . k]\right)=\pi_{2}^{*}\left(\widehat{r}_{3}[0 . . k]\right)=\left\langle\Delta^{*}, a_{2}^{*}\right\rangle$. Since $\Delta^{\prime}<\Delta$ (by definition of $\pi_{1}^{*}$ ), we have $\Delta^{*}<\Delta$, hence

$$
\widehat{r}_{3}[k+1] \in \delta_{\text {jd }}^{\widehat{\tau}_{3 b l}^{\Omega^{\text {td }}}}\left(\widehat{r}_{3}[k],\left\langle\Delta, a_{1}\right\rangle,\left\langle\Delta^{*}, a_{2}\right\rangle\right)=\left\{\delta^{\widehat{\mathcal{T}}_{3 b l}^{\Omega^{\mathrm{td}}}}\left(\widehat{r}_{3}[k],\left\langle\Delta^{*}, a_{2}\right\rangle\right)\right\} .
$$

Since $\pi_{1}^{*}, \pi_{2}^{*}$ are memoryless, we have

$$
\begin{aligned}
& \pi_{1}^{*}\left(\widehat{r}_{3}^{*}[0 . . k]\right)=\pi_{1}^{*}\left(\widehat{r}_{3}[0 . . k]\right)=\left\langle\Delta^{\prime}, \perp_{1}\right\rangle \\
& \pi_{2}^{*}\left(\widehat{r}_{3}^{*}[0 . . k]\right)=\pi_{2}^{*}\left(\widehat{r}_{3}[0 . . k]\right)=\left\langle\Delta^{*}, a_{2}\right\rangle .
\end{aligned}
$$

Thus, $\widehat{r}_{3}^{*}[k+1]$ belongs to

$$
\delta_{\mathrm{jd}}^{\widehat{\mathcal{T}}_{3 b l}^{\Omega^{\mathrm{td}}}}\left(\widehat{r}_{3}^{*}[k],\left\langle\Delta^{\prime}, \perp_{1}\right\rangle,\left\langle\Delta^{*}, a_{2}\right\rangle\right)=\left\{\delta^{\widehat{\widetilde{T}}_{3 b l}^{\Omega^{\mathrm{td}}}}\left(\widehat{r}_{3}^{*}[k],\left\langle\Delta^{*}, a_{2}\right\rangle\right)\right\}=\left\{\delta_{3 b l}^{\widehat{\widetilde{T}}_{3 b l}^{\mathrm{td}}}\left(\widehat{r}_{3}[k],\left\langle\Delta^{*}, a_{2}\right\rangle\right)\right\} .
$$

(4) The following hold.

- $\pi_{1}\left(\widehat{r}_{3}[0 . . k]\right)=\left\langle\Delta, a_{1}\right\rangle, a_{1} \neq \perp_{*}$,

- $\operatorname{Succr}_{3} \widehat{\widetilde{\tau}}^{\Omega^{\text {td }}}\left(\widehat{r}_{3}[k], \Delta\right)=$ FALSE,

- $\pi_{1}^{*}\left(\widehat{r}_{3}[0 . . k]\right)=\left\langle\Delta^{\prime}, \perp_{1}\right\rangle$, and

- $\pi_{2}^{*}\left(\widehat{r}_{3}[0 . . k]\right)=\left\langle\Delta^{*}, a_{2}^{*}\right\rangle$ with $\Delta^{*}>\Delta^{\prime}$.

We have $\pi_{2}\left(\widehat{r}_{3}[0 . . k]\right)=\left\langle\Delta^{\prime}, \perp_{2}\right\rangle$ by definition. Since $\Delta^{\prime}<\Delta$ (by definition of $\pi_{1}^{*}$ ), we have

$$
\widehat{r}_{3}[k+1] \in \delta_{\text {jd }}^{\widehat{\widetilde{T}}_{3 b l}^{\Omega^{\mathrm{td}}}}\left(\widehat{r}_{3}[k],\left\langle\Delta, a_{1}\right\rangle,\left\langle\Delta^{\prime}, \perp_{2}\right\rangle\right)=\left\{\delta^{\widehat{\widetilde{T}}_{3 b l}^{\Omega \mathrm{td}}}\left(\widehat{r}_{3}[k],\left\langle\Delta^{\prime}, \perp_{2}\right\rangle\right)\right\}
$$

Also, since $\pi_{1}^{*}$ and $\pi_{2}^{*}$ are memoryless, we have

$$
\begin{aligned}
\widehat{r}_{3}^{*}[k+1] & \in \delta_{\mathrm{jd}}^{\widehat{\widetilde{\tau}}_{3 b l}^{\mathrm{td}}}\left(\widehat{r}_{3}^{*}[k],\left\langle\Delta^{\prime}, \perp_{1}\right\rangle,\left\langle\Delta^{*}, a_{2}\right\rangle\right) \\
& =\left\{\delta^{\widehat{\widetilde{\tau}}_{3 b l}^{\mathrm{td}}}\left(\widehat{r}_{3}^{*}[k],\left\langle\Delta^{\prime}, \perp_{1}\right\rangle\right)\right\} \\
& =\left\{\delta^{\widehat{\widetilde{\tau}}_{3 b l}^{\Omega^{\mathrm{td}}}}\left(\widehat{r}_{3}[k],\left\langle\Delta^{\prime}, \perp_{1}\right\rangle\right)\right\} .
\end{aligned}
$$

Thus, $\widehat{r}_{3}[k+1]$ and $\widehat{r}_{3}^{*}[k+1]$ are the same except for perhaps the $3 b l_{1}$ component. Since Succr $_{2}^{\Omega^{\text {td }}}\left(\widehat{r}_{3}[k], \Delta^{\prime}\right)=$ FALSE (by definition of $\pi_{1}^{*}$ ), we must have $3 b l_{1}=$ FALSE in both $\widehat{r}_{3}[k+1]$ and $\widehat{r}_{3}^{*}[k+1]$. Hence $\widehat{r}_{3}[k+1]=\widehat{r}_{3}^{*}[k+1]$.

(5) The following hold. 
- $\pi_{1}\left(\widehat{r}_{3}[0 . . k]\right)=\left\langle\Delta, a_{1}\right\rangle, a_{1} \neq \perp_{*}$,

- $\operatorname{Succr} \widehat{\widetilde{T}}_{3}^{\Omega^{\text {td }}}\left(\widehat{r}_{3}[k], \Delta\right)=$ FALSE,

- $\pi_{1}^{*}\left(\widehat{r}_{3}[0 . . k]\right)=\left\langle\Delta^{\prime}, \perp_{1}\right\rangle$,

- $\pi_{2}^{*}\left(\widehat{r}_{3}[0 . . k]\right)=\left\langle\Delta^{*}, a_{2}^{*}\right\rangle$ with $\Delta^{*}=\Delta^{\prime}$

- $\delta^{\widehat{\tau}_{3 b l}^{\text {td }}}\left(\widehat{r}_{3}^{*}[k], \pi_{2}^{*}\left(\widehat{r}_{3}^{*}[0 . . k]\right)\right)=\widehat{r}_{3}^{*}[k+1]$, and

- $\delta^{\widehat{\mathcal{T}}_{3 b l}^{\text {td }}}\left(\widehat{r}_{3}^{*}[k], \pi_{1}^{*}\left(\widehat{r}_{3}^{*}[0 . . k]\right)\right) \neq \widehat{r}_{3}^{*}[k+1]$.

We have $\pi_{2}\left(\widehat{r}_{3}[0 . . k]\right)=\pi_{2}^{*}\left(\widehat{r}_{3}[0 . . k]\right)=\left\langle\Delta^{*}, a_{2}^{*}\right\rangle$ by definition. Also $\pi_{1}^{*}\left(\widehat{r}_{3}^{*}[0 . . k]\right)=$ $\pi_{1}^{*}\left(\widehat{r}_{3}[0 . . k]\right)=\left\langle\Delta^{\prime}, \perp_{1}\right\rangle ;$ and $\pi_{2}^{*}\left(\widehat{r}_{3}^{*}[0 . . k]\right)=\pi_{2}^{*}\left(\widehat{r}_{3}[0 . . k]\right)=\left\langle\Delta^{*}, a_{2}^{*}\right\rangle$ since $\pi_{1}^{*}$ and $\pi_{2}^{*}$ are both memoryless. Now, $\widehat{r}_{3}[k+1]$ belongs to $\delta_{\text {jd }}^{\widehat{\mathcal{J}}_{3 b l}^{\text {td }}}\left(\widehat{r}_{3}[k], \pi_{1}\left(\widehat{r}_{3}[0 . . k]\right), \pi_{2}\left(\widehat{r}_{3}[0 . . k]\right)\right)$, and

$$
\begin{aligned}
& \delta_{\mathrm{jd}}^{\widehat{\widetilde{T}}_{3 b l}^{\Omega^{\mathrm{td}}}}\left(\widehat{r}_{3}[k], \pi_{1}\left(\widehat{r}_{3}[0 . . k]\right), \pi_{2}\left(\widehat{r}_{3}[0 . . k]\right)\right)=\delta_{\mathrm{jd}}^{\widehat{\widetilde{\tau}}_{3 b l}^{\Omega^{\mathrm{td}}}}\left(\widehat{r}_{3}[k],\left\langle\Delta, a_{1}\right\rangle,\left\langle\Delta^{*}, a_{2}^{*}\right\rangle\right) \\
& =\left\{\delta^{\widehat{\widetilde{T}}_{3 b l}^{\Omega \mathrm{td}}}\left(\widehat{r}_{3}[k],\left\langle\Delta^{*}, a_{2}^{*}\right\rangle\right)\right\} \\
& =\left\{\delta^{\widehat{\mathcal{T}}_{3 b l}^{\Omega \mathrm{td}}}\left(\widehat{r}_{3}^{*}[k],\left\langle\Delta^{*}, a_{2}^{*}\right\rangle\right)\right\} \\
& =\left\{\widehat{r}_{3}^{*}[k+1]\right\} \text {. }
\end{aligned}
$$

Thus, we have $\widehat{r}_{3}[k+1]=\widehat{r}_{3}^{*}[k+1]$.

(6) The following hold.

- $\pi_{1}\left(\widehat{r}_{3}[0 . . k]\right)=\left\langle\Delta, a_{1}\right\rangle, a_{1} \neq \perp_{*}$,

- $\operatorname{Succr}{\widehat{\mathrm{T}^{2}}}^{\Omega^{\mathrm{td}}}\left(\widehat{r}_{3}[k], \Delta\right)=$ FALSE,

- $\pi_{1}^{*}\left(\widehat{r}_{3}[0 . . k]\right)=\left\langle\Delta^{\prime}, \perp_{1}\right\rangle$,

- $\pi_{2}^{*}\left(\widehat{r}_{3}[0 . . k]\right)=\left\langle\Delta^{*}, a_{2}^{*}\right\rangle$ with $\Delta^{*}=\Delta^{\prime}$; and

- $\delta^{\widehat{\mathrm{T}}_{3 b l} \Omega^{\mathrm{td}}}\left(\widehat{r}_{3}^{*}[k], \pi_{1}^{*}\left(\widehat{r}_{3}^{*}[0 . . k]\right)\right)=\widehat{r}_{3}^{*}[k+1]$.

We have $\pi_{2}\left(\widehat{r}_{3}[0 . . k]\right)=\left\langle\Delta^{*}, \perp_{2}\right\rangle$ by definition. Also $\pi_{1}^{*}\left(\widehat{r}_{3}^{*}[0 . . k]\right)=\pi_{1}^{*}\left(\widehat{r}_{3}[0 . . k]\right)=$ $\left\langle\Delta^{\prime}, \perp_{1}\right\rangle$; and $\pi_{2}^{*}\left(\widehat{r}_{3}^{*}[0 . . k]\right)=\pi_{2}^{*}\left(\widehat{r}_{3}[0 . . k]\right)=\left\langle\Delta^{*}, a_{2}^{*}\right\rangle$ since $\pi_{1}^{*}$ and $\pi_{2}^{*}$ are both memoryless. Then, $\widehat{r}_{3}[k+1] \in \delta_{\text {jd }}^{\widehat{\tau}_{3 b l}^{\Omega^{\mathrm{td}}}}\left(\widehat{r}_{3}[k], \pi_{1}\left(\widehat{r}_{3}[0 . . k]\right), \pi_{2}\left(\widehat{r}_{3}[0 . . k]\right)\right)$ and

$$
\begin{aligned}
\delta_{\mathrm{jd}}^{\widehat{\tau}_{3 b l} \Omega^{\mathrm{td}}}\left(\widehat{r}_{3}[k], \pi_{1}\left(\widehat{r}_{3}[0 . . k]\right), \pi_{2}\left(\widehat{r}_{3}[0 . . k]\right)\right) & =\delta_{\mathrm{jd}}^{\widehat{\Im}_{3 b l}^{\Omega^{\mathrm{td}}}}\left(\widehat{r}_{3}[k],\left\langle\Delta, a_{1}\right\rangle,\left\langle\Delta^{*}, \perp_{2}\right\rangle\right) \\
& =\left\{\delta^{\widehat{\Im}_{3 b l}^{\Omega^{\mathrm{td}}}}\left(\widehat{r}_{3}[k],\left\langle\Delta^{*}, \perp_{2}\right\rangle\right)\right\} \\
& =\left\{\delta^{\widehat{\tau}_{3 b l}^{\Omega^{\mathrm{td}}}}\left(\widehat{r}_{3}^{*}[k],\left\langle\Delta^{*}, \perp_{1}\right\rangle\right)\right\}
\end{aligned}
$$

since Succr $\widehat{\widetilde{T}}_{3}^{\Omega^{\mathrm{td}}}\left(\widehat{r}_{3}^{*}[k], \Delta\right)=\operatorname{Succr}_{3}^{\widehat{\widetilde{T}}^{\Omega^{\mathrm{td}}}}\left(\widehat{r}_{3}[k], \Delta\right)=$ FALSE, and $\Delta^{\prime}=\Delta^{*}$ and $\operatorname{Succr}_{3}{\widehat{\mathcal{T}^{\mathrm{td}}}}^{\mathrm{td}}\left(\widehat{r}_{3}^{*}[k], \Delta^{\prime}\right)=$ TRUE.

We have ,

$$
\widehat{r}_{3}^{*}[k+1]=\delta^{\widehat{\mathcal{T}}_{3 b l}^{\Omega^{\mathrm{td}}}}\left(\widehat{r}_{3}^{*}[k],\left\langle\Delta^{*}, \perp_{1}\right\rangle\right)=\delta^{\widehat{\mathcal{T}}_{3 b l}^{\Omega^{\mathrm{td}}}}\left(\widehat{r}_{3}^{*}[k],\left\langle\Delta^{\prime}, \perp_{1}\right\rangle\right)
$$


and

$$
\begin{aligned}
& \delta^{\widehat{\widetilde{T}}_{3 b l}^{\Omega^{\mathrm{td}}}}\left(\widehat{r}_{3}^{*}[k],\left\langle\Delta^{*}, \perp_{1}\right\rangle\right) \in \delta_{\mathrm{jd}}^{\widehat{\tau}_{3 b l}^{\Omega^{\mathrm{td}}}}\left(\widehat{r}_{3}^{*}[k], \pi_{1}^{*}\left(\widehat{r}_{3}^{*}[0 . . k]\right), \pi_{2}^{*}\left(\widehat{r}_{3}^{*}[0 . . k]\right)\right) \\
& =\delta_{\mathrm{jd}}^{\widehat{\tau}_{3 b l}^{\mathrm{td}}}\left(\widehat{r}_{3}^{*}[k],\left\langle\Delta^{\prime}, \perp_{1}\right\rangle,\left\langle\Delta^{*}, a_{2}^{*}\right\rangle\right) \\
& =\delta_{\mathrm{jd}}^{\widehat{\tau}_{3 b l}^{\mathrm{td}}}\left(\widehat{r}_{3}[k],\left\langle\Delta^{\prime}, \perp_{1}\right\rangle,\left\langle\Delta^{*}, a_{2}^{*}\right\rangle\right) \\
& \ni \widehat{r}_{3}[k+1]
\end{aligned}
$$

Thus, the run $\widehat{r}_{3}[0 . . k]$ can be extended such that $\widehat{r}_{3}[k+1]=\widehat{r}_{3}^{*}[k+1]$.

Thus, in all cases, we have that $\widehat{r}_{3}[0 . . k]$ can be extended to $\widehat{r}_{3}[0 . . k+1]$ according to $\pi_{1}, \pi_{2}$ such that $\widehat{r}_{3}[0 . . k+1]=\widehat{r}_{3}^{*}[0 . . k+1]$. Hence, we have $\widehat{r}_{3} \in \operatorname{Outcomes}\left(\widehat{s}, \pi_{1}, \pi_{2}\right)$ and $\widehat{r}_{3} \notin \operatorname{TimeDiv3B_{1}}(\operatorname{Parity}(\Omega))$ as $\widehat{r}_{3}^{*} \notin \operatorname{TimeDiv} 3 \mathrm{BI}_{1}(\operatorname{Parity}(\Omega))$, a contradiction since $\pi_{1}$ was assumed to be a winning strategy. Hence, we cannot have the existence of the strategy $\pi_{2}^{*}$ from which $\widehat{r}_{3}^{*}$ and $\pi_{2}$ were derived, i.e., $\pi_{1}^{*}$ is a winning strategy for player 1 from $\widehat{s}$.

\section{Completion of proof of Lemma 3.7 .}

Proof. (Continued).

We continue to show that if $\widehat{s} \in \mathrm{CPre}_{1,3}(Z)$, then $\widehat{s} \in \mathrm{CPre}_{1}(Z)$. Suppose $\widehat{s} \in \mathrm{CPre}_{1,3}(Z)$.

We can have the following cases.

(1) $\left\{\delta\left(\widehat{s},\left\langle\Delta, a_{2}\right\rangle\right) \mid\left\langle\Delta, a_{2}\right\rangle \in \Gamma_{2}(\widehat{s})\right.$ and $\operatorname{Succr}_{3}(\widehat{s}, \Delta)=$ TRUE $\} \subseteq Z$.

Consider the cardinality of the set $B=\left\{\operatorname{Reg}\left(\delta\left(\widehat{s},\left\langle\Delta, \perp_{2}\right\rangle\right) \mid\left\langle\Delta, \perp_{2}\right\rangle \in \Gamma_{2}(\widehat{s})\right)\right\}$.

- If $|B| \leq 3$ then we have

$$
\begin{aligned}
& \left\{\delta\left(\widehat{s},\left\langle\Delta, a_{2}\right\rangle\right) \mid\left\langle\Delta, a_{2}\right\rangle \in \Gamma_{2}(\widehat{s}) \text { and } \operatorname{Succr}_{3}(\widehat{s}, \Delta)=\text { TRUE }\right\} \\
= & \left\{\delta\left(\widehat{s},\left\langle\Delta, a_{2}\right\rangle\right) \mid\left\langle\Delta, a_{2}\right\rangle \in \Gamma_{2}(\widehat{s})\right\} .
\end{aligned}
$$

Hence $\widehat{s} \in \mathrm{CPre}_{1}(Z)$ in this case (when $|B| \leq 3$ ).

- Suppose $|B|>3$. Then, there exists $\Delta$ such that $\left\langle\Delta, a_{2}\right\rangle \in \Gamma_{2}(\widehat{s})$ (and hence $\left\langle\Delta, \perp_{1}\right\rangle \in$ $\left.\Gamma_{1}(\widehat{s})\right)$ with $\left|\left\{\operatorname{Reg}\left(\widehat{s}+\Delta^{\prime}\right) \mid \Delta^{\prime} \leq \Delta\right\}\right|=3$.

Consider the player-1 move $\left\langle\Delta, \perp_{1}\right\rangle$. Since $\left\langle\Delta, a_{2}\right\rangle \in \Gamma_{2}(\widehat{s})$, we must have $\left\langle\Delta, \perp_{2}\right\rangle \in$ $\Gamma_{2}(\widehat{s})$. By assumption, we have

$$
\begin{aligned}
& \delta\left(\widehat{s},\left\langle\Delta, \perp_{2}\right\rangle\right) \in Z \\
& \text { (the assumption being } \left.\left\{\begin{array}{ll}
\delta\left(\widehat{s},\left\langle\Delta, a_{2}\right\rangle\right) \mid \begin{array}{l}
\left\langle\Delta, a_{2}\right\rangle \in \Gamma_{2}(\widehat{s}) \text { and } \\
\operatorname{Succr}_{3}(\widehat{s}, \Delta)=\operatorname{TrUE}
\end{array}
\end{array}\right\} \subseteq Z\right) .
\end{aligned}
$$

Since $\Delta$ is such that $\operatorname{Succr}_{2}(\widehat{s}, \Delta)=$ FALSE, we have $\delta\left(\widehat{s},\left\langle\Delta, \perp_{2}\right\rangle\right)=\delta\left(\widehat{s},\left\langle\Delta, \perp_{1}\right\rangle\right)$ (the $3 b l_{1}$ component is false in both cases). Hence, $\delta\left(\widehat{s},\left\langle\Delta, \perp_{1}\right\rangle\right) \in Z$. Also, since

$\star\left\{\delta\left(\widehat{s},\left\langle\Delta^{\prime}, a_{2}\right\rangle\right) \mid\left\langle\Delta^{\prime}, a_{2}\right\rangle \in \Gamma_{2}(\widehat{s})\right.$ and $\operatorname{Succr}_{3}\left(\widehat{s}, \Delta^{\prime}\right)=$ TRUE $\} \subseteq Z$ and

$\star\left|\left\{\operatorname{Reg}\left(\widehat{s}+\Delta^{\prime}\right) \mid \Delta^{\prime} \leq \Delta\right\}\right|=3$

we have

$$
\left\{\delta\left(\widehat{s},\left\langle\Delta^{\prime}, a_{2}\right\rangle\right) \mid \Delta^{\prime} \leq \Delta, \text { and }\left\langle\Delta^{\prime}, a_{2}\right\rangle \in \Gamma_{2}(\widehat{s})\right\} \subseteq Z .
$$

Hence $\widehat{s} \in \mathrm{CPre}_{1}(Z)$ in this case by the second condition of $\mathrm{CPre}_{1}(Z)$ (the winning move of player 1 being $\left\langle\Delta, \perp_{1}\right\rangle$ ).

(2) There exists $\left\langle\Delta, a_{1}\right\rangle \in \Gamma_{1}(\widehat{s})$ with $a_{1} \neq \perp_{*}$ such that

(a) $\delta\left(\widehat{s},\left\langle\Delta, a_{1}\right\rangle\right) \in Z$, and

(b) $\left\{\delta\left(\widehat{s},\left\langle\Delta^{\prime}, a_{2}\right\rangle\right) \mid \Delta^{\prime} \leq \Delta\right.$, $\operatorname{Succr}_{3}\left(\widehat{s}, \Delta^{\prime}\right)=$ TRUE, and $\left.\left\langle\Delta^{\prime}, a_{2}\right\rangle \in \Gamma_{2}(\widehat{s})\right\} \subseteq Z$.

Consider the cardinality of the set $D=\left\{\operatorname{Reg}\left(\widehat{s}+\Delta^{\prime}\right) \mid \Delta^{\prime} \leq \Delta\right\}$. 
- If $|D| \leq 3$ then

$$
\begin{aligned}
& \left\{\delta\left(\widehat{s},\left\langle\Delta^{\prime}, a_{2}\right\rangle\right) \mid \Delta^{\prime} \leq \Delta, \operatorname{Succr}_{3}\left(\widehat{s}, \Delta^{\prime}\right)=\text { TRUE, and }\left\langle\Delta^{\prime}, a_{2}\right\rangle \in \Gamma_{2}(\widehat{s})\right\} \\
= & \left\{\delta\left(\widehat{s},\left\langle\Delta^{\prime}, a_{2}\right\rangle\right) \mid \Delta^{\prime} \leq \Delta, \text { and }\left\langle\Delta^{\prime}, a_{2}\right\rangle \in \Gamma_{2}(\widehat{s})\right\} .
\end{aligned}
$$

Hence $\widehat{s} \in \mathrm{CPre}_{1}(Z)$ in this case (when $|D| \leq 3$ ) by the second condition of $\mathrm{CPre}_{1}(Z$ ). - Suppose $|D|>3$. Since $\left\langle\Delta, a_{1}\right\rangle \in \Gamma_{1}(\widehat{s})$, we must have that there exists $\Delta^{\prime}<\Delta$ such that $\left\langle\Delta, \perp_{1}\right\rangle \in \Gamma_{1}(\widehat{s})$ and $\left|\left\{\operatorname{Reg}\left(\widehat{s}+\Delta^{\prime \prime}\right) \mid \Delta^{\prime \prime} \leq \Delta^{\prime}\right\}\right|=3$.

Consider the player-1 move $\left\langle\Delta^{\prime}, \perp_{1}\right\rangle$. As $\left\langle\Delta, a_{1}\right\rangle \in \Gamma_{1}(\widehat{s})$, we must have $\left\langle\Delta^{\prime}, \perp_{2}\right\rangle \in$ $\Gamma_{2}(\widehat{s})$. Also, since

$$
\left\{\delta\left(\widehat{s},\left\langle\Delta^{\prime \prime}, a_{2}\right\rangle\right) \mid \Delta^{\prime \prime} \leq \Delta, \operatorname{Succr}_{3}\left(\widehat{s}, \Delta^{\prime \prime}\right)=\text { TrUE, and }\left\langle\Delta^{\prime \prime}, a_{2}\right\rangle \in \Gamma_{2}(\widehat{s})\right\} \subseteq Z,
$$

we must have $\delta\left(\widehat{s},\left\langle\Delta^{\prime}, \perp_{2}\right\rangle\right) \in Z$. Since $\Delta^{\prime}$ is such that $\operatorname{Succr}_{2}(\widehat{s}, \Delta)=$ FALSE, we have $\delta\left(\widehat{s},\left\langle\Delta^{\prime}, \perp_{2}\right\rangle\right)=\delta\left(\widehat{s},\left\langle\Delta^{\prime}, \perp_{1}\right\rangle\right)$ (the $b l_{1}$ component is false in both cases). Hence $\delta\left(\widehat{s},\left\langle\Delta^{\prime}, \perp_{1}\right\rangle\right) \in Z$. Also, since

$\star\left\{\delta\left(\widehat{s},\left\langle\Delta^{\prime \prime}, a_{2}\right\rangle\right) \mid \Delta^{\prime \prime} \leq \Delta,\left\langle\Delta^{\prime \prime}, a_{2}\right\rangle \in \Gamma_{2}(\widehat{s})\right.$ and $\operatorname{Succr}_{3}\left(\widehat{s}, \Delta^{\prime \prime}\right)=$ TRUE $\} \subseteq Z$ and

$\star\left|\left\{\operatorname{Reg}\left(\widehat{s}+\Delta^{\prime \prime}\right) \mid \Delta^{\prime \prime} \leq \Delta^{\prime}\right\}\right|=3$,

we have

$$
\left\{\delta\left(\widehat{s},\left\langle\Delta^{\prime \prime}, a_{2}\right\rangle\right) \mid \Delta^{\prime \prime} \leq \Delta^{\prime}, \text { and }\left\langle\Delta^{\prime \prime}, a_{2}\right\rangle \in \Gamma_{2}(\widehat{s})\right\} \subseteq Z
$$

Hence $\widehat{s} \in \mathrm{CPre}_{1}(Z)$ in this case by the second condition of $\mathrm{CPre}_{1}(Z)$ (the winning move of player 1 being $\left.\left\langle\Delta^{\prime}, \perp_{1}\right\rangle\right)$.

Thus, in all cases $\widehat{s} \in \mathrm{CPre}_{1}(Z)$ whenever $\widehat{s} \in \mathrm{CPre}_{1,3}(Z)$.

\section{Proof of Lemma 3.11.}

Proof. Recall that by Equation 3.1 mentioned in Theorem 3.10, given $X=X_{1} \times\{1\} \cup X_{2} \times$ $\{2\} \subseteq S^{f}$, we have

$$
\operatorname{RegStates}\left(\operatorname{Pre}_{1}^{\mathcal{T}^{f}}\left(\operatorname{Pre}_{1}^{\mathcal{T}^{f}}(X)\right)\right)=\operatorname{RegStates}\left(\operatorname{Pre}_{1}^{\mathcal{\tau}^{f}}\left(\operatorname{Pre}_{1}^{\mathcal{\tau}^{f}}\left(X_{1} \times\{1\}\right)\right)\right)
$$

Similarly for the structure $\mathcal{T}^{f^{*}}$, given $X^{*}=X_{1_{a}}^{*} \times\left\{1_{a}\right\} \cup X_{1_{c}}^{*} \times\left\{1_{c}\right\} \cup X_{2}^{*} \times\{2\} \subseteq S^{f^{*}}$, we have

$$
\operatorname{RegStates}\left(\operatorname{Pre}_{1}^{\mathcal{T}^{*}}\left(\operatorname{Pre}_{1}^{\mathcal{T}^{*}}\left(\operatorname{Pre}_{1}^{\mathcal{T}^{f^{*}}}\left(X^{*}\right)\right)\right)\right)=\operatorname{RegStates}\left(\operatorname{Pre}_{1}^{\mathcal{T}^{*}}\left(\operatorname{Pre}_{1}^{\mathcal{T}^{f *}}\left(\operatorname{Pre}_{1}^{\mathcal{T}^{*}}\left(X_{1_{a}}^{*}\right)\right)\right)\right)
$$

Thus, it suffices to show that if $X_{1} \times\{1\} \subseteq S^{f}$ and $X_{1_{a}}^{*} \times\left\{1_{a}\right\} \subseteq S^{f^{*}}$ are such that $\operatorname{RegStates}\left(X_{1} \times\{1\}\right)=\operatorname{RegStates}\left(X_{1_{a}}^{*} \times\{1\}\right)$, then

$$
\operatorname{RegStates}\left(\operatorname{Pre}_{1}^{\mathcal{\tau}^{f}}\left(\operatorname{Pre}_{1}^{\tau^{f}}\left(X_{1} \times\{1\}\right)\right)\right)=\operatorname{RegStates}\left(\operatorname{Pre}_{1}^{\tau^{f *}}\left(\operatorname{Pre}_{1}^{\mathcal{T}^{f *}}\left(\operatorname{Pre}_{1}^{\mathcal{I}^{f^{*}}}\left(X_{1_{a}}^{*} \times\left\{1_{a}\right\}\right)\right)\right)\right) \text {. }
$$

Note that RegStates $\left(X_{1} \times\{1\}\right)=\operatorname{RegStates}\left(X_{1_{a}}^{*} \times\{1\}\right)$ implies $X_{1}=X_{1_{a}}^{*} \subseteq \widehat{S}_{\text {Reg }}$, where $\widehat{S}_{\text {Reg }}$ is the set of regions of $\widehat{\mathcal{T}}_{3 b l}^{\Omega^{\mathrm{td}}}$.

$(\Rightarrow)$ For

we show

$$
s^{f}=\langle\widehat{R}, 1\rangle \in \operatorname{Pre}_{1}^{\mathcal{T}^{f}}\left(\operatorname{Pre}_{1}^{\mathcal{T}^{f}}\left(X_{1} \times\{1\}\right)\right)
$$

$$
s^{f^{*}}=\left\langle\widehat{R}, 1_{a}\right\rangle \in \operatorname{Pre}_{1}^{\mathcal{T}^{f *}}\left(\operatorname{Pre}_{1}^{\mathcal{T}^{f *}}\left(\operatorname{Pre}_{1}^{\mathcal{T}^{f^{*}}}\left(X_{1_{a}}^{*} \times\left\{1_{a}\right\}\right)\right)\right) .
$$

Let player 1 choose to go to $\left\langle\widehat{R}, j, a_{1}, 2\right\rangle$ from $s^{f}$. We can have have two cases: 
$-a_{1}=\perp_{*}$.

If this is the move of player 1 for demonstrating that $s^{f} \in \operatorname{Pre}_{1}^{\mathcal{T}^{f}}\left(\operatorname{Pre}_{1}^{\mathcal{I f}^{f}}\left(X_{1} \times\{1\}\right)\right)$, it means that every player-2 move from $\left\langle\widehat{R}, j, a_{1}, 2\right\rangle$ goes to $X_{1} \times\{1\}$. For this to happen, we must have that in the game structure $\widehat{\mathcal{T}}_{3 b l}^{\Omega^{\text {td }}}$, for every state $\widehat{s} \in \widehat{R}$, for every player-2 move $\left\langle\Delta, a_{2}\right\rangle$ such that $\operatorname{Succr}_{3}^{\widehat{\mathcal{T}}_{3 b l}^{\Omega^{\text {td }}}}(\widehat{s}, \Delta)=$ TRUE and $\left\langle\Delta, a_{2}\right\rangle \in \Gamma_{2}(\widehat{s})$, we have $\delta\left(\widehat{s},\left\langle\Delta, a_{2}\right\rangle\right) \in \widehat{R}^{\prime}$ with $\left\langle\widehat{R}^{\prime}, 1\right\rangle \in X_{1} \times\{1\}$.

Consider $s^{f^{*}}=\left\langle\widehat{R}, 1_{a}\right\rangle$ in $S^{f^{*}}$. Let player 1 choose to go to $s_{2}^{f_{2}^{*}}=\langle\widehat{R}, 2\rangle$. Because of the restriction of player- 2 moves from $\widehat{R}$ proved above (that every player- 2 move within two successor regions must lead to a state such that the region corresponds to a region in $X_{1}$ and hence in $X_{1_{a}}^{*}$ ), we must have that in $\mathcal{T}^{f^{*}}$ every player-2 edge from $s_{2}^{f_{2}^{*}}$ leads to a state $\left\langle\widehat{R}^{\prime}, 1_{c}^{\text {dum }}\right\rangle$ such that $\widehat{R}^{\prime} \in X_{1}=X_{1_{a}}^{*}$. Let $X_{1_{c}}^{*}=X_{1}$. We now have that every player 2 edge from $s_{2}^{f^{*}}$ leads to a state in $X_{1_{c}}^{*} \times\left\{1_{c}^{\text {dum }}\right\}$. The next state in the game will obviously be in $X_{1} \times\left\{1_{a}\right\}=X_{1_{a}}^{*} \times\left\{1_{a}\right\}$. Thus, $s^{f^{*}}=\left\langle\widehat{R}, 1_{a}\right\rangle \in \operatorname{Pre}_{1}^{\mathcal{T}^{f^{*}}}\left(\operatorname{Pre}_{1}^{\mathcal{I f}^{*}}\left(\operatorname{Pre}_{1}^{\mathcal{T}^{f^{*}}}\left(X_{1_{a}}^{*} \times\left\{1_{a}\right\}\right)\right)\right)$ if the first move of player 1 corresponds to $\perp_{*}$.

$-a_{1} \neq \perp_{*}$.

This case can have two subcases according to the available move for player 2 .

- This subcase corresponds to the case where player 2 does not "allow" the player-1 move in a corresponding stage in $\widehat{\mathcal{T}}_{3 b l}^{\Omega^{\text {td }}}$. We must have fact 1: that for every $\widehat{s} \in$ $\widehat{R}$, for every player-2 move $\left\langle\Delta, a_{2}\right\rangle$ such that $\left\langle\Delta, a_{2}\right\rangle \in \Gamma_{2}^{\widehat{\mathcal{T}}_{3 b l}^{\Omega^{\mathrm{td}}}(\widehat{s})}$ and $\mid\left\{\operatorname{Reg} \widehat{s}+\Delta^{\prime} \mid\right.$ $\left.\Delta^{\prime} \leq \Delta\right\} \mid \leq j+1$, we have that $\delta^{\widehat{\mathcal{T}}_{3 b l}^{\text {td }}}\left(\widehat{s},\left\langle\Delta, a_{2}\right\rangle\right) \in \widehat{R}^{\prime}$ such that $\operatorname{Reg}\left(\widehat{R}^{\prime}\right) \in X_{1}$.

- This subcase corresponds to the case where player 2 "allows" the player- 1 move. We must have fact 2: that for every $\widehat{s} \in \widehat{R}$, there exists a player-1 move $\left\langle\Delta, a_{1}\right\rangle$ such that $\left\langle\Delta, a_{1}\right\rangle \in \Gamma_{1}^{\widehat{\tau}_{3 b l}^{\Omega^{\text {td }}}}(\widehat{s})$ and $\left|\left\{\operatorname{Reg} \widehat{s}+\Delta^{\prime} \mid \Delta^{\prime} \leq \Delta\right\}\right|=j+1$, we have that $\delta^{\widehat{\mathcal{T}}_{3 b l}^{\Omega^{\mathrm{td}}}}\left(\widehat{s},\left\langle\Delta, a_{1}\right\rangle\right) \in \widehat{R}^{\prime}$ such that $\operatorname{Reg}\left(\widehat{R}^{\prime}\right) \in X_{1}$.

Let $X_{1_{c}}^{*}=X_{1}=X_{1_{a}}^{*}$. Consider $s^{f^{*}}=\left\langle\widehat{R}, 1_{a}\right\rangle$ in $S^{f^{*}}$. Let player 1 choose to go to $\langle\widehat{R}, j, 2\rangle$. we show player 1 can ensure going to $X_{1_{a}}^{*} \times\left\{1_{1_{a}}\right\}$ with this choice. Player 2 can do two things from $\langle\widehat{R}, j, 2\rangle$.

- Player 2 decides to "not allow" the player-1 move in a corresponding stage in $\widehat{\mathcal{T}}_{3 b l}^{\Omega^{\mathrm{td}}}$. This corresponds to player 2 taking an edge to a state $\left\langle\widehat{R}^{\prime}, 1_{c}^{\text {dum }}\right\rangle$ in $\mathcal{T}_{\Omega}^{f^{*}}$ from $s^{f^{*}}$ which can happen if for every $\widehat{s} \in \widehat{R}$, there exists a player-2 move $\left\langle\Delta, a_{2}\right\rangle$ such that $\left\langle\Delta, a_{2}\right\rangle \in \Gamma_{2}^{\widehat{\tau}_{3 b l}^{\text {td }}}(\widehat{s})$ and $\left|\left\{\operatorname{Reg} \widehat{s}+\Delta^{\prime} \mid \Delta^{\prime} \leq \Delta\right\}\right| \leq j+1$. By fact 1 , we must have that for every such $\left\langle\Delta, a_{2}\right\rangle$, we must have $\delta^{\Upsilon_{3 b l}^{\text {td }}}\left(\widehat{s},\left\langle\Delta, a_{2}\right\rangle\right) \in \widehat{R}^{\prime}$ such that $\operatorname{Reg}\left(\widehat{R}^{\prime}\right) \in X_{1}$. Hence, every "not allow" player-2 edge in $\mathcal{T}_{\Omega}^{f *}$ from $s^{f^{*}}$ leads a state in $X_{1_{c}}^{*} \times\left\{1_{c}^{\text {dum }}\right\}$, the next state from which will lie in $X_{1_{a}}^{*} \times\left\{1_{a}\right\}$.

- Player 2 decides to "allow" the player-1 move in a corresponding stage in $\widehat{\mathcal{T}}_{3 b l}^{\Omega^{\text {td }}}$. This corresponds to player 2 taking the edge from $s^{f^{*}}$ in $\mathcal{T}_{\Omega}^{f^{*}}$ to the state $\left\langle\widehat{R}, j, 1_{c}\right\rangle$. From $\left\langle\widehat{R}, j, 1_{c}\right\rangle$, because of fact 2 , player 1 can pick the edge corresponding to the action $a_{1}$ which will lead it to a state in $X_{1} \times\left\{1_{a}\right\}=X_{1_{a}}^{*} \times\left\{1_{a}\right\}$. 
Thus, if $a_{1} \neq \perp_{*}$, player 1 has a strategy to go from $s^{f^{*}}$ to $X_{1_{a}}^{*} \times\left\{1_{a}\right\}$ in three steps. $s^{f^{*}}=\left\langle\widehat{R}, 1_{a}\right\rangle \in \operatorname{Pre}_{1}^{\mathcal{T}^{f^{*}}}\left(\operatorname{Pre}_{1}^{\mathcal{\tau}^{* *}}\left(\operatorname{Pre}_{1}^{\mathcal{T}^{f^{*}}}\left(X_{1_{a}}^{*} \times\left\{1_{a}\right\}\right)\right)\right)$.

Hence in both cases, we have that

$$
s^{f^{*}}=\left\langle\widehat{R}, 1_{a}\right\rangle \in \operatorname{Pre}_{1}^{\mathcal{T}^{f^{*}}}\left(\operatorname{Pre}_{1}^{\mathcal{T}^{f^{*}}}\left(\operatorname{Pre}_{1}^{\mathcal{T}^{f^{*}}}\left(X_{1_{a}}^{*} \times\left\{1_{a}\right\}\right)\right)\right) .
$$

$(\Leftarrow)$ For

we show

$$
s^{f^{*}}=\left\langle\widehat{R}, 1_{a}\right\rangle \in \operatorname{Pre}_{1}^{\mathcal{T}^{f *}}\left(\operatorname{Pre}_{1}^{\mathcal{T}^{f *}}\left(\operatorname{Pre}_{1}^{\mathcal{T}^{f *}}\left(X_{1_{a}}^{*} \times\left\{1_{a}\right\}\right)\right)\right)
$$

$$
s^{f}=\langle\widehat{R}, 1\rangle \in \operatorname{Pre}_{1}^{\mathcal{T}^{f}}\left(\operatorname{Pre}_{1}^{\mathcal{T}^{f}}\left(X_{1} \times\{1\}\right)\right) .
$$

The following cases arise depending on the move of player 1 from $s^{f^{*}}$ which witnesses

$$
s^{f^{*}} \in \operatorname{Pre}_{1}^{\mathcal{J}^{f^{*}}}\left(\operatorname{Pre}_{1}^{\mathcal{T}^{f^{*}}}\left(\operatorname{Pre}_{1}^{\mathcal{T}^{f^{*}}}\left(X_{1_{a}}^{*} \times\left\{1_{a}\right\}\right)\right)\right) .
$$

- Player 1 moves to $\langle\widehat{R}, 2\rangle$ (this corresponds to a move $a_{1}=\perp_{*}$ in $\mathcal{T}$ ).

This means that every player 2 move from $\langle\widehat{R}, 2\rangle$ goes to $X_{1_{a}}^{*} \times\left\{1_{a}\right\}$. Since $X_{1_{a}}^{*}=X_{1}$, we have $\langle\widehat{R}, 1\rangle \in \operatorname{Pre}_{1}^{\mathcal{T}^{f}}\left(\operatorname{Pre}_{1}^{\mathcal{T}^{f}}\left(X_{1} \times\{1\}\right)\right)$ if the first move of player 1 from $s^{f^{*}}$ corresponds to $\perp_{*}$.

- Player 1 moves to $\langle\widehat{R}, j, 2\rangle$. This case has two subcases.

- Suppose we have fact 3 : every player 2 move from $\langle\widehat{R}, j, 2\rangle$ to $\left\langle\widehat{R}^{\prime}, 1_{c}^{\text {dum }}\right\rangle$ is such that $\widehat{R}^{\prime} \in X_{1_{a}}^{*}$.

Consider the situation in $\mathcal{T}^{f}$ where player 1 moves to some $\left\langle\widehat{R}, j, a_{1}, 2\right\rangle$ with $a_{1} \neq$ $\perp_{*}$ and player 2 takes an edge corresponding to it not allowing player 1 in $\mathcal{T}$. Because of fact 3 , all such edges must go to $X_{1} \times\{1\}$, no matter the action $a_{1}$.

- Suppose we have fact 4: every player 2 move from $\langle\widehat{R}, j, 2\rangle$ to $\left\langle\widehat{R}, j, 1_{c}\right\rangle$ is such that $\left\langle\widehat{R}, j, 1_{c}\right\rangle \in \operatorname{Pre}_{1}^{\mathcal{T}^{f^{*}}}\left(X_{1_{a}}^{*} \times\left\{1_{a}\right\}\right)$. This means that in $\mathcal{T}$, from any state in $\widehat{R}$, if player 1 is allowed to let time elapse to get to the $j$-th successor from $\widehat{R}$, then it can take a discrete action $a_{1}$ such that the resultant state will be in some region in $X_{1}=X_{1_{a}}^{*}$. Thus, there must exist a player-1 action $a_{1}$ such that from the state $\left\langle\widehat{R}, j, a_{1}, 2\right\rangle$ in $\mathcal{T}^{f}$, all player-2 edges, which correspond to it allowing a move in $\mathcal{T}$, end up in $X_{1} \times\{1\}$.

Because of the two above subcases, if the witness for

$$
s^{f^{*}} \in \operatorname{Pre}_{1}^{\mathcal{T}^{f *}}\left(\operatorname{Pre}_{1}^{\mathcal{T}^{f *}}\left(\operatorname{Pre}_{1}^{\mathcal{T}^{f *}}\left(X_{1_{a}^{*}}^{*} \times\left\{1_{a}\right\}\right)\right)\right)
$$

in the structure $\mathcal{T}^{f^{*}}$ is a move to $\langle\widehat{R}, j, 2\rangle$, then in the structure $\mathcal{T}^{f}$, there exists a move of player 1 to a state $\left\langle\widehat{R}, j, a_{1}, 2\right\rangle$ such that $\left\langle\widehat{R}, j, a_{1}, 2\right\rangle \in \operatorname{Pre}_{1}^{\mathcal{T}^{f}}\left(X_{1} \times\{1\}\right)$.

Thus in both cases of player-1 winning moves in $\mathcal{T}^{f^{*}}$ from $s^{f^{*}}$, we have the existence of a player-1 move from $s^{f}$ which witnesses that $s^{f}=\langle\widehat{R}, 1\rangle \in \operatorname{Pre}_{1}^{\mathcal{T}^{f}}\left(\operatorname{Pre}_{1}^{\mathcal{T}^{f}}\left(X_{1} \times\{1\}\right)\right)$. 


\subsection{Proofs of Section 4, Completion of analysis of Example 4.9}

Example 4.9 (continued). Let the game start from the location $l^{0}$. In a run $r$, let $t_{1}^{j}$ and $t_{2}^{j}$ be the times when the $a_{1}^{1}$-th transition and the $a_{1}^{2}$-th transitions respectively are taken for the $j$-th time. The constraints are $t_{1}^{j}-t_{1}^{j-1} \leq 1$ and $t_{2}^{j}-t_{2}^{j-1}>1$. If the game cycles infinitely often in between $l^{0}$ and $l^{1}$ we must also have that for all $j \geq 0, t_{1}^{j+1} \geq$ $t_{2}^{j} \geq t_{1}^{j}$. Conversely, we also have that if this condition holds then we can construct an infinite time divergent cycle of $l^{0}, l^{1}$ for some suitable initial clock values. Observe that $t_{i}^{j}=t_{i}^{0}+\left(t_{i}^{1}-t_{i}^{0}\right)+\left(t_{i}^{2}-t_{i}^{1}\right)+\cdots+\left(t_{i}^{j}-t_{i}^{j-1}\right)$ for $i \in\{1,2\}$. We need

$$
t_{1}^{m+1}-t_{2}^{m}=\left(t_{1}^{m+1}-t_{1}^{m}\right)+\sum_{j=1}^{m}\left\{\left(t_{1}^{j}-t_{1}^{j-1}\right)-\left(t_{2}^{j}-t_{2}^{j-1}\right)\right\}+\left(t_{1}^{0}-t_{2}^{0}\right) \geq 0 \text { for all } m \geq 0 .
$$

Rearranging, we get the requirement

$$
\sum_{j=1}^{m}\left\{\left(t_{2}^{j}-t_{2}^{j-1}\right)-\left(t_{1}^{j}-t_{1}^{j-1}\right)\right\} \leq\left(t_{1}^{m+1}-t_{1}^{m}\right)+\left(t_{1}^{0}-t_{2}^{0}\right) .
$$

Consider the initial state $\left\langle l^{0}, x=y=0\right\rangle$. Let $t_{1}^{0}=1, t_{2}^{0}=1.1, t_{1}^{j}-t_{1}^{j-1}=1, t_{2}^{j}-t_{2}^{j-1}=$ $1+10^{-(j+1)}$. We have

$\sum_{j=1}^{m}\left\{\left(t_{2}^{j}-t_{2}^{j-1}\right)-\left(t_{1}^{j}-t_{1}^{j-1}\right)\right\} \leq \sum_{j=1}^{\infty} 10^{-(j+1)}=10^{-2} * \frac{1}{0.9} \leq 1-0.1=\left(t_{1}^{m+1}-t_{1}^{m}\right)+\left(t_{1}^{0}-t_{2}^{0}\right)$.

Thus, we have an infinite time divergent trace with the given values. Hence $\left\langle l^{0}, x=\right.$ $y=0\rangle \in \operatorname{WinTimeDiv}_{1}^{\mathcal{T}}\left(\square\left(\neg l^{3}\right)\right)$. It can also be similarly seen that $\left\langle l^{0}, x=y=1\right\rangle \in$ WinTimeDiv $_{1}\left(\square\left(\neg l^{3}\right)\right.$ ) (taking $t_{1}^{0}=0$ and $t_{2}^{0}=0.1$ ). We also have $\left\langle l^{0}, x=y=1\right\rangle \notin$ $\operatorname{RobWinTimeDiv}_{1}\left(\square\left(\neg l^{3}\right)\right)$ as player 1 would have to take the first $a_{1}^{1}$ transition immediately from this state.

We now show $\left\langle l^{0}, x=y=0\right\rangle \in \operatorname{RobWinTimeDiv}_{1}\left(\square\left(\neg l^{3}\right)\right)$. Consider

$$
\begin{aligned}
t_{1}^{0} & \in[0.9,1], t_{1}^{j}-t_{1}^{j-1} \in\left[1-10^{-(j+1)}, 1\right] \\
t_{2}^{0} & \in[1.05,1.1] \\
t_{2}^{j}-t_{2}^{j-1} & \in\left[1+0.5 * 10^{-(j+1)}, 1+10^{-(j+1)}\right] .
\end{aligned}
$$

We have

$$
\begin{aligned}
\sum_{j=1}^{m}\left\{\left(t_{2}^{j}-t_{2}^{j-1}\right)-\left(t_{1}^{j}-t_{1}^{j-1}\right)\right\} & \leq \sum_{j=1}^{m} 10^{-(j+1)}-\left(-10^{-(j+1)}\right) \\
& \leq 2 * \sum_{j=1}^{\infty} 10^{-(j+1)} \\
& =2 * 10^{-2} * \frac{1}{0.9}
\end{aligned}
$$


We also have $\left(t_{1}^{m+1}-t_{1}^{m}\right)+\left(t_{1}^{0}-t_{2}^{0}\right) \geq 1-10^{-(m+2)}+(0.9-1.1) \geq 0.7$. Thus, we have

$$
\begin{aligned}
\sum_{j=1}^{m}\left\{\left(t_{2}^{j}-t_{2}^{j-1}\right)-\left(t_{1}^{j}-t_{1}^{j-1}\right)\right\} & <2 * 10^{-2} * \frac{1}{0.9} \\
& <0.7 \\
& \leq\left(t_{1}^{m+1}-t_{1}^{m}\right)+\left(t_{1}^{0}-t_{2}^{0}\right) .
\end{aligned}
$$

This shows that we can construct an infinite cycle in between $l^{0}$ and $l^{1}$ for all the values in our chosen intervals, and hence that $\left\langle l^{0}, x=y=0\right\rangle \in \operatorname{RobWinTimeDiv}_{1}\left(\square\left(\neg l^{3}\right)\right)$.

We next show that $\left\langle l^{0}, x=y=0\right\rangle \notin \mathrm{JRWinTimeDiv} \varepsilon_{1}^{\varepsilon_{\mathrm{j}}, \varepsilon_{\mathrm{r}}}\left(\square\left(\neg l^{3}\right)\right)$ for any $\varepsilon_{\mathrm{j}}>0$. Observe that for any objective $\Phi$, we have JRWinTimeDiv ${ }_{1}^{\varepsilon_{\mathrm{j}}, \varepsilon_{\mathrm{r}}}(\Phi) \subseteq \operatorname{JRWinTimeDiv}_{1}^{\varepsilon_{\mathrm{j}}, 0}(\Phi)$. Let $\varepsilon_{\mathrm{j}}=\epsilon$ and let $\varepsilon_{\mathrm{r}}=0$. Consider any player-1 strategy $\pi_{1}$, for $\epsilon$-jitter 0 -response time boundedrobust winning, that makes the game cycle in between $l^{0}$ and $l^{1}$. Player 2 then has a strategy which "jitters" the player- 1 moves by $\epsilon$. Thus, the player- 1 strategy $\pi_{1}$ can only propose $a_{1}^{1}$ moves with the value of $x$ being less than or equal to $1-\epsilon$ (else the jitter would make the move invalid). Thus, player 2 can ensure that $t_{1}^{j}-t_{1}^{j-1} \leq 1-\epsilon$ for all $j$ for some run (since $x$ has the value $t_{1}^{j}-t_{1}^{j-1}$ when $a_{1}^{1}$ is taken for the $j$-th time for $j>0$ ). We then have that for any player-1 strategy that is a candidate for $\epsilon$-jitter 0 -response time bounded-robust winning, player 2 has a strategy such that for some resulting run, we have $t_{1}^{j}-t_{1}^{j-1} \leq 1-\epsilon$ and $t_{2}^{j}-t_{2}^{j-1}>1$. Thus, $\sum_{j=1}^{m}\left\{\left(t_{2}^{j}-t_{2}^{j-1}\right)-\left(t_{1}^{j}-t_{1}^{j-1}\right)\right\}>m * \epsilon$, which can be made arbitrarily large for a sufficiently large $m$ for any $\epsilon$ and hence greater than $\left(t_{1}^{m+1}-t_{1}^{m}\right)+\left(t_{1}^{0}-t_{2}^{0}\right) \leq 1+\left(t_{1}^{0}-t_{2}^{0}\right)$ for any initial values of $t_{1}^{0}$ and $t_{2}^{0}$. This violates the requirement for an infinite $l^{0}, l^{1}$ cycle. Thus, $\left\langle l^{0}, x=y=0\right\rangle \notin \operatorname{JRWinTimeDiv}_{1}^{\epsilon, 0}\left(\square\left(\neg l^{3}\right)\right)$ for any $\epsilon>0$. 